\title{
SELF-STUDY MANUAL ON OPTICAL RADIATION MEASUREMENTS
}

\author{
Part I - Concepts \\ Chapter 10
}




\section{NATIONAL BUREAU OF STANDARDS}

The National Bureau of Standards' was established by an act of Congress on March 3, 1901. The Bureau's overall goal is to strengthen and advance the Nation's science and technology and facilitate their effective application for public benefit. To this end, the Bureau conducts research and provides: (1) a basis for the Nation's physical measurement system, (2) scientific and technological services for industry and government, (3) a technical basis for equity in trade, and (4) technical services to promote public safety. The Bureau's technical work is performed by the National Measurement Laboratory, the National Engineering Laboratory, and the Institute for Computer Sciences and Technology.

THE NATIONAL MEASUREMENT LABORATORY provides the national system of physical and chemical and materials measurement; coordinates the system with measurement systems of other nations and furnishes essential services leading to accurate and uniform physical and chemical measurement throughout the Nation's scientific community, industry, and commerce; conducts materials research leading to improved methods of measurement, standards, and data on the properties of materials needed by industry, commerce, educational institutions, and Government; provides advisory and research services to other Government agencies; develops, produces, and distributes Standard Reference Materials; and provides calibration services. The Laboratory consists of the following centers:

\section{Absolute Physical Quantities ${ }^{2}$ - Radiation Research - Chemical Physics - Analytical Chemistry - Materials Science}

THE NATIONAL ENGINEERING LABORATORY provides technology and technical services to the public and private sectors to address national needs and to solve national problems; conducts research in engineering and applied science in support of these efforts; builds and maintains competence in the necessary disciplines required to carry out this research and technical service; develops engineering data and measurement capabilities; provides engineering measurement traceability services; develops test methods and proposes engineering standards and code changes; develops and proposes new engineering practíces; and develops and improves mechanisms to transfer results of its research to the ultimate user. The Laboratory consists of the following centers:

Applied Mathematics - Electronics and Electrical Engineering ${ }^{2}$ - Manufacturing Engineering - Building Technology - Fire Research - Chemical Engineering ${ }^{2}$

THE INSTITUTE FOR COMPUTER SCIENCES AND TECHNOLOGY conducts research and provides scientific and technical services to aid Federal agencies in the selection, acquisition, application, and use of computer technology to improve effectiveness and economy in Government operations in accordance with Public Law 89-306 (40 U.S.C. 759), relevant Executive Orders, and other directives; carries out this mission by managing the Federal Information Processing Standards Program, developing Federal ADP standards guidelines, and managing Federal participation in ADP voluntary standardization activities; provides scientific and technological advisory services and assistance to Federal agencies; and provides the technical foundation for computer-related policies of the Federal Government. The Institute consists of the following centers:

Programming Science and Technology-Computer Systems Engineering.

'Headquarters and Laboratories at Gaithersburg, MD, unless otherwise noted;

mailing address Washington, DC 20234.

${ }^{2}$ Some divisions within the center are located at Boulder, CO 80303. 


\section{Self-Study Manual on \\ Optical Radiation Measurements: \\ Part I - Concepts, Chapter 10}

Fred E. Nicodemus, Editor

Chapter 10. Introduction to Coherence in Radiometry, by John B. Shumaker

Preparation and publication of this Technical Note has been supported by funding from agencies listed on pages $v$ and $v i$.

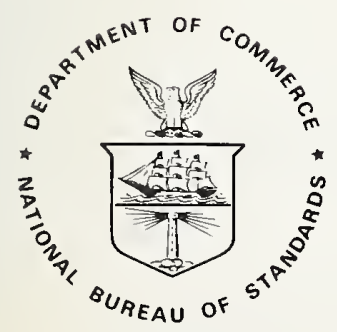

U.S. DEPARTMENT OF COMMERCE, Malcolm Baldrige, Secretary NATIONAL BUREAU OF STANDARDS, Ernest Ambler, Director 
National Bureau of Standards Technical Note 910-6

NatI. Bur. Stand. (U.S.), Tech. Note 910-6, 69 pages (March 1983)

CODEN: NBTNAE

U.S. GOVERNMENT PRINTING OFFICE

WASHINGTON: 1983

For sale by the Superintendent of Documents, U.S. Government Printing Office, Washington, D.C. 20402 Price $\$ 5.00$

(Add 25 percent for other than U.S. mailing) 
Preparation of the NBS Self-Study Manual on Optical Radiation Measurements is an important part of our effort to meet the needs of the National Measurement System with respect to the measurement of optical electromagnetic radiation. Significant needs exist for measurements with uncertainties of about one percent, but the uncertainties actually achieved are often 5 to 10 percent, or even more. These relatively poor accuracies, as compared to those in many other types of physical measurements, result to a great extent from the multidimensional character of optical radiation; radiant power is distributed and may vary with position, direction, wavelength, polarization, and time. Measurement results are also affected by various instrumental and environmental parameters. In addition, many of those who make measurements of optical radiation have little or no training or experience in this field and are limited in the amount of time they can devote to acquiring the needed information, understanding, and proficiency with measurement techniques. Moreover, there are few schools that offer courses in radiometry, and there are almost no adequate texts or references dealing with this entire subject.

The idea of producing a Self-Study Manual at NBS to try to fill some of this void was developed by one of us (HJK) in the latter part of 1973. Detailed planning got under way in the summer of 1974 when a full-time Editor (FEN) was appointed. The first nine chapters were published in a series of four NBS Technical Notes (910- series) up to June 1979. However, subsequent progress was slowed by administrative uncertainties and problems that affected the availability of authors, causing several changes in scheduling, and that finally ended all direct NBS funding for the Project on September 30, 1979. Welcome transfers of funds from a number of other federal agencies were obtained to carry the Project to september 30 , 1980 , with the hope that it could then be restored to the NBS budget. When that failed to materialize, outside funding was again obtained to september 30, 1981 (through FY 81).

Meanwhile the Program Director (HJK) retired at the end of May 1981, and the Editor on September 29, 1981. However, remaining FY 81 funds were used for a grant to Catholic University of America (CUA), with whom NBS negotiated a cooperative agreement under which CUA hired the full-time Editor (FEN) who continues, as before, at NBS (same address and telephone), working closely with the new Project Head (JBS) there. CUA is also engaging the services of three other former NBS employees: Dr. Kostkowski as part-time consultant throughout the Project and as author or co-author of two more chapters, Mr. Richmond as author or co-author of two more chapters, and Dr. Venable as author of one more chapter, as outlined in more detail below.

This Technical Note 910-6, with Chapter 10 of Part I--Concepts, is the first departure from classical (geometrical- or ray-optics) radiometry in this Manual. From the beginning, due primarily to the rapid growth in the use of lasers, there has been a clear and increasingly urgent need for a treatment of the radiometry of coherent radiation, where geometrical (ray) optics is often an inadequate approximation. However, when Dr. Shumaker started, almost three years ago, to look into the possibility of writing a chapter on coherence in radiometry, it was still not clear whether the basic theory on which this chapter could be based had been developed sufficiently to make it feasible. Fortunately, the recent rapid progress in this area has taken place and we are delighted to be able, at last, to respond to a number of repeated requests for the material in this chapter.

Our aim for the entire Manual has been to provide a comprehensive tutorial treatment that is complete enough for self instruction. That is what is meant in the title by "self-study"; the Manual does not contain explicitly programmed learning steps as that term often connotes. In addition, through detailed, yet concise, chapter summaries, the Manual is designed to serve also as a convenient reference source. Those already familiar with a topic should turn immediately to the summary at the end of the appropriate chapter. They can determine from that summary what, if any, of the body of the chapter they want to read for more details.

The material in the Manual is presented at the level of a college graduate in science or engineering, but even for those with facility in college mathematics and a first course in physics, it's not at all easy reading in spite of our best efforts at clarity and simplicity. This is an unavoidable result of the primary aim ("to make one-percent measurements commonplace") coupled with the fact that it must serve the needs of so many different fields, including astronomy and astrophysics, mechanical heat-transfer engineering, illumination engineering, photometry, meteorology, photo-biology and photo-chemistry, optical pyrometry, remote sensing, military infrared applications, etc. Chapter 10 is probably one of the most difficult, since it is the first attempt by anyone, so far as we know, to present the radiometric implications of coherence as they affect those making such measurements. We've done our best to make it as simple and readable as possible, but it just isn't a simple subject. Most readers will probably find that they can't follow it all on first reading, even taking it slowly and carefully. But don't be discouraged. Sleep on it and come back to it, more than once if necessary. We wish we could suggest a number of other sources to consult, a technique that often helps in such situations. However, the whole viewpoint and approach needed here is so different from that of most of the other publications on coherence (that aren't primarily concerned with making radiometric measurements) that it may be difficult to reconcile them with the material here. Furthermore, enough care and thought have gone into the preparation 
of this chapter to convince us that it is sound and that, if you'll persevere and give enough time for each new idea to become familiar to you, you'll find that it's an adequate and useful introduction.

Apparently it is very difficult for those who have not been directly involved to realize the full implications of the situation just discussed. Each of us tends to think of radiometry and radiometric measurements in terms of our own immediate experiences and requirements. What each of us would like to have is a set of simple, carefully designed procedures for making our own particular measurements, with appropriate cautions concerning likely sources of error. However, the next reader wants the same thing, but for entirely different measurements. The desired radiometric quantities to be measured are different, the instrumentation is different, the ambient conditions are different -- the possible ways in which significant differences may exist, in terms of the radiation parameters (position, direction, spectrum, time or frequency of modulation or fluctuation, and polarization) as well as instrumental and environmental parameters, are so numerous that any attempt to cover them all with a "cookbook" treatment of specific measurement procedures would be impossibly unwieldy and could never be completed within any conceivable budget limitations short of utopia. The only way in which we can hope to effectively assist every reader who needs to make one-percent measurements is to provide you, the reader, with material which, with sufficient effort on your part, will help you to develop sufficient understanding and grasp of basic principles to solve your own particular measurement problems. That's why we have concentrated on the basic material of Part I--Concepts and why it is not easy reading.

As an exception, we are currently publishing, in NBS TN 910-5, just one chapter of Part III--Applications (see list of chapters, past, present and future, below). It gives a detailed account of some very difficult high-accuracy field measurements of ultraviolet solar terrestrial spectral irradiance, with frequent references to applicable portions of the earlier chapters of Part I--Concepts to illustrate how they can be used to achieve improved measurement results. It also illustrates clearly the complexities of such high-accuracy measurements that make it impossible for anyone other than you yourself to determine the details of how best to make your particular measurements with your particular instruments and constraints and for your particular objectives.

The present chapter on coherence is, like Chapter 6 on polarization, an attempt to present the subject as it pertains to radiometric measurements. There are many interesting and highly important features of coherence phenomena, as well as of polarization phenomena. However, we are not concerned with them here, as such, but only to the extent that they affect the measurement of the flow of power or flux in a beam of electromagnetic optical radiation.

The basic approach and focal point of the treatment in this Manual is the measurement equation, first introduced in detail but limited to the radiation parameters of position, direction, and spectrum, in Chapter 5. We believe that every measurement problem should be addressed with such an equation, relating the quantity desired to the data obtained, through a detailed characterization of the instruments used and the radiation field observed, in terms of all of the relevant parameters. These parameters always include the radiation parameters (listed above), as well as environmental and instrumental parameters peculiar to each measurement configuration. The objective of the Manual is to develop the basic concepts required so that the reader will be able to use this measurement-equation approach. It is our belief that this is the only way that uncertainties in the measurement of optical radiation can generally be limited to one, or at most a few, percent.

The original, overall plan for the Manual organized it into three Parts: Part I--Concepts, Part II--Instrumentation, and Part III--Applications. That was our rather ambitious plan when we started out; limitations of support and available resources, particularly available authors, have determined how much, or how little, we could accomplish. So far, as indicated above, we have concentrated on Part I--Concepts. The Part I chapters already published are:

1. Introduction, by F. E. Nicodemus, H. J. Kostkowski, and A. T. Hattenburg

2. Distribution of Optical Radiation with Respect to Position and Direction--Radiance, by F. E. Nicodemus and H. J. Kostkowski

3. Spectral Distribution of Optical Radiation, by F. E. Nicodemus and H. J. Kostkowski

4. More on the Distribution of Optical Radiation with Respect to Position and Direction, by Fred E. Nicodemus

5. An Introduction to the Measurement Equation, by Henry J. Kostkowski and Fred E. Nicodemus

6. Distribution of Optical Radiation with Respect to Polarization, by John B. Shumaker 
7. The Relative Spectral Responsivity and Slit-Scattering Function of a Spectroradiometer, by Henry J. Kostkowski

8. Deconvolution, by John B. Shumaker

9. Physically Defining Measurement-Beam Geometry by Using Opaque Barriers, by Fred E. Nicodemus.

Chapters 1, 2, and 3 were in TN 910-1; 4 and 5 in TN 910-2; 6 in TN 910-3; and 7, 8, and 9 in TN 910-4. Now in publication, as mentioned above, is TN 910-5 with Part III--Applications:

Chapter 1. Measurement of Solar Terrestrial Spectral Irradiance in the

Ozone Cut-off Region, by Henry J. Kostkowski, Robert D. Saunders,

John F. Ward, Charles H. Popenoe, and A.E.S. Green.

And in the present TN 910-6, we return to Part I--Concepts:

10. Introduction to Coherence in Radiometry, by John B. Shumaker.

Contingent on the availability of outside funding support, we hope that, during the next two to three years, we can continue the current arrangements with Catholic University of America as described above and complete the following additional chapters of Part I--Concepts:

Distribution of Optical Radiation with Respect to Time, by Fred E. Nicodemus

Linearity Considerations and Calibrations, by John B. Shumaker

Spectrophotometry, by William H. Venable, Jr.

Blackbody Radiation and Temperature Scales, by Joseph C. Richmond and/or Henry J. Kostkowski

Physical Photometry, by A. T. Hattenburg and/or Fred E. Nicodemus

Thermal Radiation Properties of Matter, by Joseph C. Richmond

Spectroradiometry of Spectral Lines, by Henry J. Kostkowski.

As stated above, the measurement-equation approach is central to the entire Manual and the material presented in each chapter needs to be related to the approach introduced in Chapter 5. Depending on how well this is accomplished in each chapter, there may be need at the and to have a final summary chapter for this purpose, tying up loose ends and putting the whole Part I into perspective. Also, examples of various categories of environmental and instrumental parameters and their significance could be usefully presented and discussed. But it's too soon to evaluate this need at present.

Incidentally, in preparing material for the Manual, we have had the pleasure of rediscovering the fact that the best way to learn anything is to try to teach it to someone else. The exercise of preparing tutorial material for such wide general application has required us to analyze our own measurement activities in a different way, broadening our understanding and resulting in improved methods and more accurate results. Note that all references to measurement accuracy or uncertainty in this preface are concerned not only with precision (relating to the repeatability of measurement results) but also with accuracy (relating to agreement with the "truth" which, while unknowable in the last analysis, is approximated by analyses and estimates based on the widest possible experience, including agreement with measurements of the same quantities by others, particularly when they have used different instrumentation and methods of measurement).

ACKNOWLEDGMENTS. Several experts who read an early draft version of this chapter on coherence offered many valuable suggestions. We are particularly grateful to: William H. Carter, J. Christopher Dainty, Jack D. Gaskill, Arwind S. Marathay, and Emil Wolf.

As indicated above, we have been entirely supported by funds from other federal agencies since september 30, 1979. This is one of the first publications since that date, so it is one of our first opportunities to publicly acknowledge that most welcome support from the following:

DoD, CCG (tri-service Calibration Coordination Group), Working Group for IR/Laser (Mr. Major L. Fecteau - Army; Mr. Robert Hinebaugh - Air Force; Dr. Felix Schweizer - Navy)

Naval Weapons Center, China Lake, California (Dr. Jon A. Wunderlich)

USAF, Air Staff, Pentagon, Washington, D.C. (Lt. Col. John E. Dunkle) through USAF, Space Division, Los Angeles, California (Maj. Robert Chadbourne)

Naval Air Systems Command, Washington, D.C. (Mr. E. T. Hooper, Mr. Robert C. Thyberg, Mr. Frank Daspit, and Mr. Webb K. Whiting) 
USAF, Avionics Laboratory, Wright-Patterson AFB, Ohio (Dr. Paul J. Huffman and Mr. William J. Cannon)

Defense Advanced Research Projects Agency (DARPA), Arlington, Virginia (Dr. Robert W. Fossum through Dr. John Meson)

U.S. Army, BMD Advanced Technology Center, Huntsville, Alabama (Dr. William O. Davies)

NASA Hq., Environmental Observation Program, Washington, D.C. (Dr. L. C. Greenwood and Dr. S. G. Tilford)

Solar Energy Research Institute (SERI), Golden, Colorado (Dr. Keith Masterson)

U.S. Army, Office of Missile Electronic Warfare, Electronic Warfare Laboratory, White Sands Missile Range, New Mexico (Mr. Thomas A. Atherton)

Office of Naval Research, Arlington, Virginia (Dr. William J. Condell)

U.S. Army, Night-Vision and Electro-Optics Laboratory, Ft. Belvoir, Virginia (Mr. Wayne Grant).

There have been many others who, in these days of tight budgets, were not able to contribute financial support to this project when they wanted to but who helped very substantially in other ways. They recommended potential sources of financial support and urged those who were able to do so to participate. Just their words of encouragement and of constructive criticism were tremendously helpful. To all of these we are most sincerely grateful.

We are also deeply indebted to a great many individuals for invaluable "feedback", concerning the technical content of the Manual, that has encouraged us and has helped us to put these chapters together more effectively. We renew our strong invitation to all readers to submit comments, criticisms, and suggestions. In particular, we would welcome illustrative examples and problems from as widely different areas of application as possible.

We are especially grateful to Mrs. Betty Castle for the skillful and conscientious effort that produced the excellent typing and layout of still another difficult text.

Fred E. Nicodemus, Editor

John B. Shumaker, Project Head

Henry J. Kostkowski, Consultant

August 1982 
Concepts .

Chapter 10 .

Introduction to Coherence in Radiometry . . . . . . .

In this CHAPTER . . . . . . . . . . . . . . . . . . . 1

INTRODUCTION • . . . . . . . . . . . . . . . . . . . 1

THE CROSS-SPECTRAL DENSITY FUNCTION • . . . . . . . . . • . . 4

CROSS-SPECTRAL DENSITY and RADIOMETRIC QUANTITIES • . . • . . 8

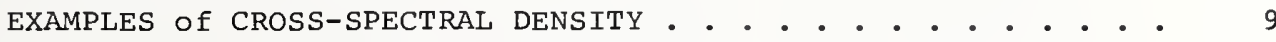

The PROPAGATION of CROSS-SPECTRAL DENSITY . . . . . . . • . . 13

MODELS for the CROSS-SPECTRAL DENSITY

FUNCTION -- QUASIHOMOGENEITY . • • . . • • . • . . . . . . . 25

The EFFECTS of IMAGING or FOCUSING OPTICS . . . . . . . . . . 29

COHERENCE EFFECTS ON A MONOCHROMATOR

SLIT-SCATTERING FUNCTION • . . . . . . . . . . . . . . . 37

MORE On IMAGING -- PUPIL FUNCTIONS and ABERRATIONS . . . . . . 44

THE CLASSICAL RADIOMETRIC QUANTITIES . • . • . . • . . . • . . 47

POLARIZATION • • • • . . . . . . . . . . . . . . . . 53

SUMMARY Of CHAPTER 10 . . . . . . . . . . . . . . . 55

References . . . . . . . . . . . . . . . . 59

\section{List of Figures}

10.1 Alternating regions of constructive (C-clear) and destructive (D-dotted) interference in the radiation field about two point (small) sources. These regions will wriggle and writhe as the frequencies (or amplitudes or phases) of the oscillators change . . . . . .

10.2 A train of plane waves incident on the $X-Y$ plane at an angle $\theta_{0}$ from the $\mathrm{z}$-axis in the $\mathrm{X}-\mathrm{Z}$ plane and with a Gaussian amplitude distribution . . . . . . . . . . . . . . . . . . . . . . . .

10.3 The propagation of cross-spectral density $\mathrm{W}_{\lambda}\left(\mathrm{P}_{1}, \mathrm{P}_{2}\right)$, assumed known for all pairs of points $P_{1}$ and $P_{2}$ in one cross section of a beam (source plane). Optical path lengths $\mathbf{s}_{1}$ and $\mathbf{s}_{2}$ extend to corresponding points $\mathrm{P}_{1}^{\prime}$ and $\mathrm{P}_{2}^{\prime}$ in a second plane where the cross-spectral density is wanted (detector plane). . . . . . . . . . . . .

10.4 A typical field point $P^{\prime}\left(x^{\prime}, y^{\prime}, z^{\prime}\right)$ is shown at a distance $R$ from the origin and a distance $s$ from a point $P(x, y, 0)$ in the source

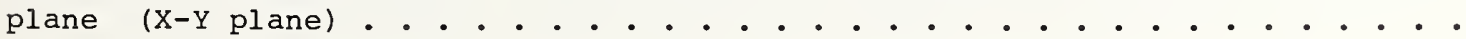


10.6 (a) Cross-spectral density propagation from source plane to intermediate optical element (mirror)

(b) Cross-spectral density propagation from intermediate optical element (mirror) to detector plane

(c) Cross-spectral density propagation from source plane through intermediate optical element (lens) to detector plane . . . . .

10.7 Relative distribution in the $\mathrm{x}$-direction of fluence rate in the image of a $50[\mu \mathrm{m}]-w i d e$, uniform, incoherent source for various lens aperture widths. Plots of the part of eq. (10.116) within curly brackets for $\lambda=514[\mathrm{~nm}], \quad \mathrm{a}=25[\mu \mathrm{m}], \mathrm{R}=\mathrm{R}^{\prime \prime}=0.8[\mathrm{~m}]$, and $\longrightarrow a^{\prime}=0.01[\mathrm{~m}] ;-a^{\prime}=0.02[\mathrm{~m}] ;-a^{\prime}=0.04[\mathrm{~m}]$;

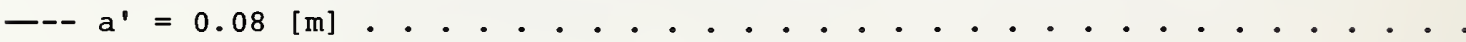

10.8 Relative distribution in the $\mathrm{x}$-direction of fluence rate in the image of a $50[\mu \mathrm{m}]-w i d e$, uniform, coherent source for various angles of incidence of the beam. Plots of the double integral of eq. (10.119) for the same parameter values as in figure 10.7 except $a^{\prime}=0.0375[\mathrm{~m}]$ and $A_{0}=1 .-\theta=0.00$ [rad]; $-\theta_{0}=0.03[\mathrm{rad}] ;-\theta_{0}=0.04[\mathrm{rad}] ;--\theta_{0}=0.05[\mathrm{rad}] . . .$.

10.9 Relative distribution in the $\mathrm{x}$-direction of fluence rate in the image of a $50[\mu \mathrm{m}]-w i d e$, uniform, partially coherent source at normal incidence for various degrees of partial coherence. Plots of the double integral of eq. (10.124) for the same parameter values as in figure 10.8 and area normalized, in the interval shown, to the area under the $\theta_{0}=0$ curve in figure 10.8. $a_{0} / R_{0}=0.00$ (coherent); $-a_{0} / R_{0}=0.04 ;-a_{0} / R_{0}=0.045 ;--a_{0} / R_{0}=0.05 ;$ - $a_{0} / R_{0}=\infty$ (incoherent . . . . . . . . . . . . . . . . . . . . . . . .

10.10 Two samples of the relative distribution in the $\mathrm{x}$-direction of

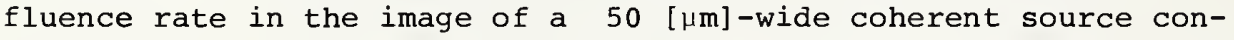
sisting of 1 [ $\mathrm{mm}$ ] cells with amplitude and phase constant within each cell but random from cell to cell . . . . . . . . . . . . . . . . . 
Part I. Concepts

This is the sixth in a series of Technical Notes (910-) entitled "Self-Study Manual on Optical Radiation Measurements". It contains Chapter 10 of Part I of this Manual. Additional chapters will continue to be published, similarly, as they are completed. The Manual is a comprehensive tutorial treatment of the measurement of optical radiation that is complete enough for self instruction. Detailed chapter summaries make it also a convenient authoritative reference source.

In this chapter we introduce the reader to the basic field quantity of the theory of partial coherence, the cross-spectral density function, and show how it is used to describe radiation fields. We discuss the propagation of cross-spectral density along a beam and illustrate this propagation with calculations of diffraction effects in a number of simple aperturing and imaging examples. For instance, we treat in considerable detail one of the most common radiometric situations in which coherence effects can manifest themselves, the measurement of the slit-scattering function of a monochromator. Among other things, this treatment shows that laser illumination of a monochromator entrance slit must be nearly on-axis to avoid serious slit-scattering function distortions. Finally, we present the relationships between the cross-spectral density function and the classical radiometric quantities, such as spectral radiance.

Key Words: coherence; cross-spectral density; incoherence; interference; partial coherence; radiometry; spectroradiometry; wave optics.

Chapter 10. Introduction to Coherence in Radiometry

by John B. Shumaker

In this CHAPTER. We attempt to provide an introduction to the theory of partial coherence and to its significance for radiometry through some illustrations of its application to radiometric situations. We begin with the cross-spectral density function which is used to describe the radiometric field, including phase relationships, much as the spectral radiance function describes the field in classical radiometry. A few examples of this function are presented and discussed. Then we give the equation for the propagation of cross-spectral density from one surface (such as a source) to another (such as a detector). This equation is then applied to such radiometric situations as the irradiation of a surface, image formation, and the calculation of the slit-scattering function of a monochromator. Although, in principle, the cross-spectral density function is measurable in detail, it is usually more practical to assume some simple functional form or model for this function; some common models are given. Finally, we discuss the definition of the classical radiometric quantities, such as radiance, in terms of the cross-spectral density function and we mention briefly the extension of the elementary theory presented in this chapter to a vector theory that includes polarization.

INTRODUCTION. In treating the fundamentals of radiometry (optical radiation measurements) in the first nine chapters of Part I of this Manual, we have so far limited ourselves to the domain of geometrical or ray optics, where it is assumed that the dimensions of all optical components are large compared to a wavelength $\left(\sim 10^{-6}[\mathrm{~m}]\right)$ and all times are long compared to a period $\left.\left(\sim 10^{-14}\right)[s]\right)$, etc. ${ }^{1}$ Diffraction and interference phenomena, which are best understood in terms of wave optics, rather than ray optics, have so far been ignored. We

\footnotetext{
${ }^{1}$ Unit symbols are all enclosed in square brackets to emphasize dimensionality and encourage routine checks of unit-dimension consistency, as discussed in reference [10.1].
} 


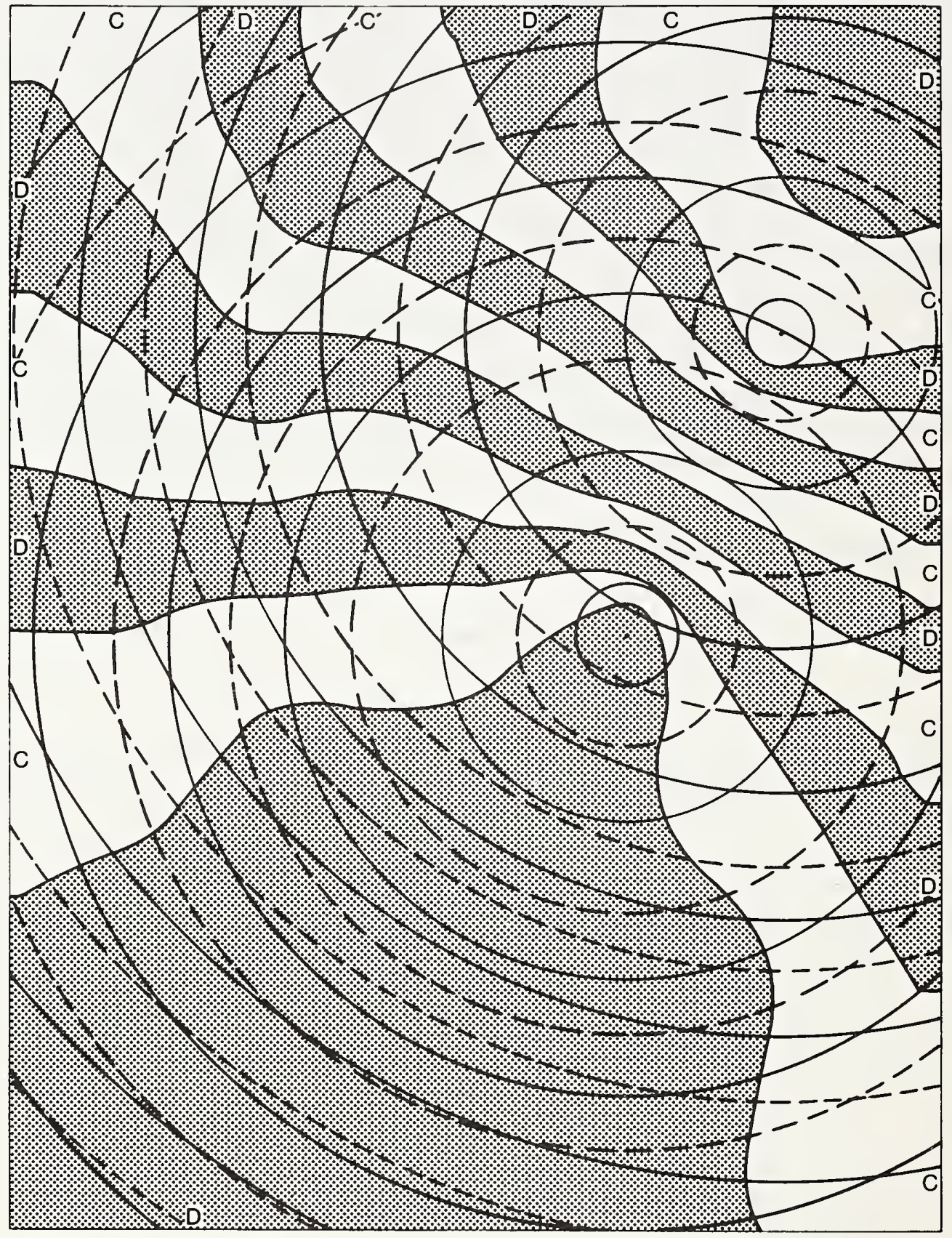

Figure 10.1. Alternating regions of constructive (C-clear) and destructive (D-dotted) interference in the radiation field about two point (small) sources. These regions will wriggle and writhe as the frequencies (or amplitudes or phases) of the oscillators change. 
are, however, very conscious of the fact that there is a rapidly increasing interest in measurements of laser radiation which, being highly coherent, require a wave-optics treatment. Also a rapidly increasing amount of material has appeared in the literature on the theory of optics and its significance for radiometry. Most of this material, however, has been at a theoretical level where it is not too useful for those actually engaged in making radiometric measurements. It is the objective of this chapter to begin to bridge this gap and to present the results of some of these theoretical treatments in a form that will make clear their application to radiometric measurements.

The theory of partial coherence is filled with complex details and some readers may find that they "loose the thread" at times. If so, the summary at the end of the chapter may prove helpful to bring more clearly into focus the pattern of the overall treatment. In addition, some recent publications [10.2] ${ }^{1}$ to [10.8] provide further reading and references to the growing literature on partial coherence. Our treatment will be found to be different in some respects from these largely theoretical references due to our preoccupation with radiometric measurements and to our recognition throughout this Manual that practical definitions of measurable radiometric quantities (intensity, radiance, etc., and crossspectral density, introduced below) must include a dependence upon the many-dimensional resolution interval of the measuring radiometer (e.g. spectral pass-band). This latter difference is minor, being limited to the purely formal connection between the electromagnetic field and radiometric quantities. Nevertheless it has proven difficult to cite specific references for the ideas and equations presented here and we have done so only in rare instances. However, we wish to emphasize that almost nothing in this chapter is original; it can all be found somewhere in the literature in some form.

We will begin by trying to describe the nature of a classical radiometric field at the microscopic level. When the fields from separate oscillators are superimposed, the electric and magnetic) field vectors add, producing a resultant field vector, and it is the effect that this resultant field has on a detector which we ultimately interpret as a measure of incident radiant power. If two point (very small) sources have roughly similar frequencies and are oriented so that their fields have the same polarization, then there will be regions of space, whose minimum dimension is at least half a wavelength, where the electric field vectors will lie in the same direction at the same time and will tend to reinforce each other over a time period of at least a cycle or two of the optical frequency ( $10^{-14}$ [s]). Such regions will obviously alternate with regions where the field vectors lie in opposite directions and tend to cancel during this same time period (see figure 10.1). In general, if the two oscillators are changing frequency or amplitude or phase these various regions will move about and change shape and size with time. When these regions of reinforcement and cancellation are large enough and move slowly enough to be detected by the eye or by an optical instrument, we call the phenomenon interference and we say that the light from the two sources possesses coherence. If no such regions of alternating radiant power are discerned by the best instruments then we say that the field is incoherent and that there is no interference. Presumably the interference (coherence) is still there but the temporal or spatial response of the instrumentation is inadequate to resolve it. Notice that the regions of positive and negative reinforcement in figure 10.1 generally increase in width with increasing distance from the source. This suggests that, although the field at one point may be judged incoherent, it may show distinct coherence effects at points more distant from the source. As we shall see, this increase in the degree of coherence with distance from the source is quite real; the light from the most incoherent-seeming source,

\footnotetext{
${ }^{1}$ Figures in brackets indicate literature references listed at the end of this Technical Note.
} 
such as a star, is highly coherent at astronomical distances, as is evidenced by stellar interferometer measurements [10.2].

Diffraction is the name associated with departures from regular (geometrical- or rayoptics) propagation of light. The spreading of light after passage through a pinhole or slit is an example of diffraction. Beyond the slit or pinhole the light beams from the different parts of the opening interfere producing the diffraction effect. Thus, interference and diffraction are essentially the same and originate in the wave nature of light. All of these phenomena are included in the theory of partial coherence which is the subject of this chapter.

Fortunately in most optical radiation measurement situations diffraction or interference effects are quite negligible so that it is adequate to use the 'simple' geometrical optics and classical radiometry which has been the subject of previous chapters. When these effects cannot be neglected, their quantitative treatment by the theory of partial coherence can be quite concisely stated [eq. (10.36), p. 13, for example]. However, the physical detail and mathematical complications which are required to handle any but the most trivial optical situations force the adoption of a formidable chain of physical idealizations and mathematical and numerical approximations. This is not to say that an accurate calculation could not be carried out for a real problem of sufficient importance but that an approximate calculation coupled with suitable experimental measurements will usually be more efficient and convincing. Thus, we will approach the treatment of coherence as providing a guide to measurement problem areas and to estimates of errors and corrections to geometrical optics when coherence effects are small. In this spirit we will frequently make assumptions and approximations in this chapter which would otherwise be intolerable.

THE CROSS-SPECTRAL DENSITY FUNCTION. In this chapter we will treat what is known as the scalar theory of coherence: we deal with only a single polarization component of the electromagnetic field -- say the $x$-component. In a later section we will discuss briefly the complications introduced by the vector nature of the electromagnetic field. Now we want to attempt to describe the connection between the electromagnetic field and the quantity known as the cross-spectral density (or the mutual spectral density). The cross-spectral density can be regarded as a radiometric quantity somewhat more basic than spectral radiance in that it provides an even more detailed specification of the radiometric field. Our discussion of the connection between the cross-spectral density function and the electromagnetic field is not intended to be rigorous but merely to provide, perhaps, some quick insight into the nature of this function. (For a rigorous derivation of this relationship we recommend especially reference [10.3].) It would be entirely sufficient to take the phenomenological view that it is convenient to define such a radiometric field quantity in terms of which, as we shall see, not only can all of the classical radiometric quantities be expressed but also most interference and diffraction effects as well. Everything of significance to radiometry can be viewed in this way without any concern for a more basic picture of the meaning of the cross-spectral density function.

Let us denote the $\mathrm{x}$-component of the electromagnetic field as a function of time at a point $P$ by $E(P, t)$. We imagine that $E(P, t)$ will be roughly sinusoidal (as a function of time) but with a continuously changing amplitude and frequency. The optical frequencies we are concerned with are in the neighborhood of $10^{11}$ to $10^{16}$ [Hz]. Although someday sensors may be developed which are fast enough to measure $E(P, t)$, at present all radiometric detectors are square-law detectors whose output is at least approximately proportional to the integral of the square of $E(P, t)$ over a response time of $10^{-9}$ [s] or more. The square of a sinusoidal field is proportional to the magnitude of the poynting vector of electromagnetic theory, and the Poynting vector is associated with the energy per second 
which crosses a unit area perpendicular to the vector direction. Thus, a proportionality relationship is generally assumed between the incident radiometric power and the response of a detector or the square of the electric field: ${ }^{1}$

$$
F_{t}\left(P_{0}\right)=\frac{k}{\Delta t \cdot \Delta a} \int_{\Delta t} \int_{\Delta S} E^{2}(P, t) \cdot d P \cdot d t
$$

where $F_{t}\left(P_{0}\right)$ is the measurable average fluence rate (see Chapter 4, p. 17 [10.9]) and $\mathrm{K}$ is some proportionality constant. The time required for a measurement is $\Delta t$ and $\Delta a$ is the cross-sectional area $\left(=\pi r^{2}\right)$ of a small opaque spherical detector surface $\Delta S \quad\left(=4 \pi r^{2}\right)$ centered at the point of interest $P_{0}{ }$. The integrations are over the detector surface and measurement time. If the electromagnetic field passes through a narrow pass-band spectral filter centered at wavelength $\lambda_{0}$ before being detected then the radiometric quantity incident on the detector is the average spectral fluence rate over the spectral pass band $\Delta \lambda$ of the filter:

$$
F_{t, \lambda}\left(P_{0}, \lambda_{0}\right)=\frac{k}{\Delta t \cdot \Delta a} \int_{\Delta t} \int_{\Delta S} \frac{E^{2}\left(P, t ; \lambda_{0}\right)}{\Delta \lambda} \cdot d P \cdot d t,
$$

where $E\left(P, t ; \lambda_{0}\right)$ is the filter-modified field at the point $P$. As we have mentioned in previous chapters a radiometric quantity, such as spectral fluence rate, at a particular point, wavelength, and instant should be identified with an extrapolation from a sequence of measurements of successively narrower spatial, spectral and temporal resolution. But, because none of these instrument resolution intervals can ever be taken as zero due, ultimately, to the noise-imposed limit of the minimum energy required for meaningful measurement, there is in practice a set of smallest values for $\Delta t, \Delta a$, and $\Delta \lambda$ consistent with the best

\footnotetext{
${ }^{1}$ We will frequently specify the function arguments $x, y, z$ simply as $P$. That is, $E(P) \equiv E(x, y, z)$. An integral such as in eq. (10.1) containing dP means that the point $P$ explores the region defined by the integration limit. In eq. (10.1), since $\Delta S$ is a spherical surface, the integration over $\mathrm{P}$ is understood to be 2-dimensional and could be written out in full as
}

$$
\int_{\Delta S} E^{2}(P) \cdot d P \equiv \int_{0}^{2 \pi} \int_{0}^{\pi} E^{2}(r, \theta, \phi) \cdot r^{2} \cdot \sin \theta \cdot d \theta \cdot d \phi \cdot
$$

Usually in this chapter $P$ will be confined to an area in the $X-Y$ plane, and an integral would again be understood to be 2-dimensional. Thus

$$
\int_{\Delta \mathrm{A}} \cdot \cdot \mathrm{dP} \equiv \int_{\Delta \mathrm{A}} \int \cdot \cdot \mathrm{dx} \cdot \mathrm{dy}
$$

or an equivalent in polar coordinates. This shorthand use of $P$ and dP will generally be confined to generalizations and concepts; equations intended for numerical evaluation will be written out fully. Also note that we use the parameter subscript to denote a derivative: $F_{t} \equiv d F / d t ; \quad F_{t, \lambda} \equiv d^{2} F /(d t \cdot d \lambda) ;$ etc., as in [10.10]. 
foreseeable technology which can be used to provide an adequate, unique definition of radiometric quantities. For the purpose of establishing a correspondence between electromagnetic theory and radiometry then we perform a suitable average as in eq. (10.4) of electromagnetic fields over resolution intervals $\Delta t, \Delta a$, and $\Delta \lambda$ with these values and must assume that radiometric quantities have been measured at, or extrapolated to, this same resolution. Normally, in radiometric practice (intercomparing beams by measurements with the same instrumentation) these resolution intervals cancel out so that their values are largely irrelevant.

It is customary in the treatment of oscillating quantities in physics and engineering to introduce complex variables. ${ }^{1}$ Since a complex variable requires two numbers for its specification this notation provides a compact way to keep track of both amplitude and phase information with a single variable. Whatever the form of $E(t)$ it can be expressed by a Fourier integral ${ }^{2}$

$$
E(t)=\int_{0}^{\infty} A(v) \cdot \cos [\gamma(v)-2 \pi v t] \cdot d v,
$$

\footnotetext{
${ }^{1}$ We will use the following properties of complex numbers: A complex number, $v$, can always be expressed as the sum of a real part, $a$, and the product of $i$ by an imaginary part, $b$, where $a$ and $b$ are ordinary real numbers:

$$
\begin{aligned}
& V \equiv a+i \cdot b \\
& \operatorname{Re}(V) \equiv a, \quad \operatorname{Im}(V) \equiv b
\end{aligned}
$$

and $i$, of course, satisfies $i^{2}=-1$. Equivalently $v$ can be expressed as

$$
V=R \cdot \exp (i \cdot \theta)=R \cdot(\cos \theta+i \cdot \sin \theta)
$$

where $R$ and $\theta$ are also ordinary real numbers related to $a$ and $b$ by

$$
R=\sqrt{a^{2}+b^{2}}, \quad \tan \theta=b / a \text {. }
$$

$R$ is called the modulus of $V$, and $\theta$ is called the argument or phase of $V$. The complex conjugate of $\mathrm{V}$ denoted by $V^{*}$ is obtained by replacing $i$ by $-i$ throughout any expression for $v$ which involves only real quantities and $i$

$$
\begin{aligned}
V^{*} & \equiv a-i \cdot b \\
& =R \cdot \exp (-i \cdot \theta) .
\end{aligned}
$$

We also define

$$
|\mathrm{V}| \equiv \mathrm{R}=\sqrt{\mathrm{V} \cdot \mathrm{V}^{\star}}
$$

${ }^{2}$ The signs of the phase terms $\gamma(\nu)$ and $2 \pi \nu t$ in eqs. (10.10) and (10.11) are entirely arbitrary; the evolution of $E(t)$ or $V(t)$ with time is the same for either sign. The present choice leads to agreement with most of the coherence literature. 
where $A(\nu)$ and $\gamma(\nu)$ are uniquely defined by the function $E(t)$, and $\nu$ is the frequency. The complex field function $V(t)$ which represents $E(t)$ is then obtained by replacing the cosine function by a complex exponential function

$$
V(t)=\int_{0}^{\infty} A(\nu) \cdot \exp [i \gamma(\nu)-2 \pi i v t] \cdot d \nu
$$

Thus the real part of $V(t)$ is identically $E(t)$. The imaginary part can be regarded as a mathematical device for coding the phase information. The substitution of $V(t)$ for $E(t)$ in equations which are linear in $E(t)$ presents no problem; one can always take the real part of such equations to recover valid physical relationships. However, equations quadratic in $E(t)$ require the substitution of $\frac{1}{2} V^{*}(t) \cdot V(t)$ for $E^{2}(t)$ and are valid only in the limit of long time averages. Fortunately in radiometry this limit is always reached; the measurement time $\Delta t$ is long compared to the natural time scales of the field $1 / \nu$ and $1 / \Delta \nu \sim \frac{1}{v} \cdot \frac{\lambda}{\Delta \lambda}$. In accord with this customary notation then we will write eq. (10.4) in complex notation as

$$
F_{t, \lambda}\left(P_{0}, \lambda_{0}\right)=\frac{k}{2 \Delta t \cdot \Delta a} \int_{\Delta t} \int_{\Delta S} \frac{V^{*}\left(P, t_{i} \lambda_{0}\right) \cdot V\left(P, t_{i} \lambda_{0}\right)}{\Delta \lambda} \cdot d P \cdot d t,
$$

with the real part of $V\left(P, t ; \lambda_{0}\right)$ being simply the electric field $E\left(P, t ; \lambda_{0}\right)$ at the point $P$ and time $t$, as transmitted by a very good spectral filter of passband $\Delta \lambda$ centered near $\lambda_{0}$. The complete function $V$, including its imaginary part, is constructed from $E$ by decomposing $\mathrm{E}$ into its Fourier components and reassembling as indicated by eqs. (10.10) and (10.11). $V^{*}$ is the complex conjugate of $V$. Actually we never perform a detailed mathematical averaging over time and area because we never know $V(P, t ; \lambda)$ for optical frequencies. The significance of eq. (10.12) is primarily to show that a definite connection exists between the electromagnetic field and a measurable radiometric quantity. For this reason it is sufficient to write eq. $(10.12)$ as

$$
F_{t, \lambda}\left(P_{0}, \lambda_{0}\right)=\frac{1}{\Delta \lambda} \cdot\left\langle V^{*}\left(P, t_{i} \lambda_{0}\right) \cdot V\left(P, t_{i} \lambda_{0}\right)\right\rangle
$$

where the angular brackets represent the averaging over measurement time and area. We have also taken the liberty of incorporating the proportionality constant $\mathrm{k} / 2$ into the definition of $\mathrm{V}$ since we will never have occasion to assign numerical values or unit dimensions to $\mathrm{V}$.

In eq. (10.13) we have shown explicitly the dependence of the fluence rate and the complex field $\mathrm{V}(\mathrm{P})$ upon the field point of interest in order that we can now introduce a generalization of eq. $(10.13)$ by allowing the quantities $V^{*}$ and $V$ to refer to two different space points:

$$
\mathrm{W}_{\lambda}\left(\mathrm{P}_{1}, \mathrm{P}_{2}, \lambda\right)=\frac{1}{\Delta \lambda} \cdot\left\langle\mathrm{V}^{*}\left(\mathrm{P}_{1}+\Delta \mathrm{P}, \mathrm{t} ; \lambda\right) \cdot \mathrm{V}\left(\mathrm{P}_{2}+\Delta \mathrm{P}, \mathrm{t} ; \lambda\right)\right\rangle .
$$

The angular bracket average in eq. (10.14) consists of an average over a small time interval and over identical small areas about $\mathrm{P}_{1}$ and $\mathrm{P}_{2}$ as the small displacement $\Delta \mathrm{P}$ covers the 
measurement resolution area. ${ }^{1}$ We assume that the radiometric field is steady in the sense that, although $\mathrm{V}$ depends upon time strongly, the average over measurement time implied by the angular brackets in eq. (10.14) changes slowly, if at all, from one measurement time to the next so that we can ignore any time dependence for $W_{\lambda}\left(P_{1}, P_{2}, \lambda\right)$. We will also hereafter usually not display the dependence upon wavelength; we will be dealing exclusively with spectral radiometric quantities and will leave it understood that they depend upon $\lambda$. $W_{\lambda}\left(P_{1}, P_{2}\right)$, which may be complex, is called the cross-spectral density function. Usually we apply this function to pairs of points lying in a particular surface such as a source plane, an image plane, or an entrance aperture. It is immaterial whether the points $\mathrm{P}_{1}$ and $\mathrm{P}_{2}$ are points in a real physical object or are simply two points in a field.

CROSS-SPECTRAL DENSITY and RADIOMETRIC QUANTITIES. When the two points coincide the crossspectral density function reduces to the spectral fluence rate

$$
W_{\lambda}\left(P_{1}, P_{1}\right)=F_{t, \lambda}\left(P_{1}\right) \text {. }
$$

This quantity, the spectral fluence rate, or its integral, fluence rate, is usually called the optical intensity, or simply the intensity, in the coherence literature. The relationship between spectral fluence rate from a distant small source and the quantity known in radiometry as the spectral radiant intensity of that source is

$$
F_{t, \lambda}(P)=I_{\lambda}(\theta, \phi) / R^{2}
$$

where $\mathrm{R}$ and $\theta, \phi$ are the distance and direction from the source whose spectral radiant intensity is $I_{\lambda}$ in the direction of the point where the description of the field is desired. The spectral radiant intensity $I_{\lambda}$ is a property of a (possibly hypothetical) source while the spectral fluence rate $F_{t, \lambda}(P)$ is a measure of the radiant power concentration in the field at the point $P$.

Finally, for completeness, we present a relationship between the cross-spectral density in a plane and the spectral radiance in that plane: ${ }^{2}$

\footnotetext{
${ }^{1}$ By analogy to the definition of fluence rate the area average is, strictly speaking, a single surface integral in which $\Delta \mathrm{P}$ moves over a small sphere so that $\mathrm{P}_{1}+\Delta \mathrm{P}$ and $\mathrm{P}_{2}+\Delta \mathrm{P}$ simultaneously describe surfaces centered on $\mathrm{P}_{1}$ and $\mathrm{P}_{2}$. The measurement resolution area is the area of a great circle of this small sphere.
}

${ }^{2}$ Where it may improve clarity, we will sometimes separate the coordinates of $P_{1}$ and $P_{2}$ by a semicolon. For example

$$
W\left(P_{1}, P_{2}\right) \equiv W\left(x_{1}, y_{1}, z_{1} ; x_{2}, y_{2}, z_{2}\right)
$$

Also we will frequently omit the $z$ coordinate in such expressions when the points are confined to a plane $\mathbf{z}=$ const. In this case we distinguish different planes by coordinates labeled by primes (') and use notations like

$$
W\left(x_{1}, y_{1} ; x_{2}, y_{2}\right) \equiv W\left(x_{1}, y_{1}, z ; x_{2}, y_{2}, z\right)
$$

and

$$
W^{\prime}\left(x_{1}^{\prime}, y_{1}^{\prime} ; x_{2}^{\prime}, y_{2}^{\prime}\right) \equiv W\left(x_{1}^{\prime}, y_{1}^{\prime}, z^{\prime} ; x_{2}^{\prime}, y_{2}^{\prime}, z^{\prime}\right) .
$$




$$
\begin{aligned}
L_{\lambda}(x, y, \theta, \phi) & =\frac{\cos \theta}{\lambda^{2}} \cdot \iint w_{\lambda}(x+\xi / 2, y+n / 2 ; x-\xi / 2, y-n / 2) \\
& \cdot \exp \left[-\frac{2 \pi i}{\lambda} \cdot(\xi \cdot \sin \theta \cdot \cos \phi+\eta \cdot \sin \theta \cdot \sin \phi)\right] \cdot d \xi \cdot d n
\end{aligned}
$$

Here, $\theta$ and $\phi$ are the usual polar coordinates of the viewing direction. The integrations are over the entire cross-section of the beam or over the source if the surface is a source plane. We will return to a discussion of this equation later in this chapter. ${ }^{1}$ For the present we will confine our attention to the spectral fluence rate because of its simple, direct connection to the cross-spectral density function. As is evident from the discussion of fluence rate in Chapter 4 [10.9], this radiometric quantity is closely related to spectral irradiance. At most points in most fields of interest in radiometry the fluence rate will have a larger value than the irradiance by a factor, of the order of unity, which changes very slowly, if at all, with position in the field and with wavelength. So, for the kind of accuracy with which we are concerned in this chapter, the two quantities are virtually interchangeable.

EXAMPLES of CROSS-SPECTRAL DENSITY. In coherence theory the cross-spectral density function can be regarded as a basic field entity much as spectral radiance is the basic field entity of conventional geometric-optics radiometry. Unfortunately the physical meaning of the cross-spectral density function is more abstract than that of radiance. The cross-spectral density embodies simultaneously information about the radiometric field strength and information about the phase correlations at all pairs of points. Needless to say, it is not a function which one can tabulate or graph easily, depending, as it does upon at least $\mathrm{x}_{1}, \mathrm{y}_{1}, \mathrm{x}_{2}, \mathrm{y}_{2}$, and $\lambda$ and having both real and imaginary components.

To gain some insight into the nature of the cross-spectral density function let us examine the behavior of $V(P, t)$ for a typical optical field and calculate $W_{\lambda}\left(P_{1}, P_{2}\right)$ from the definition, eq. (10.14). First let us assume that the field is generated by a single distant oscillator. Since we are particularly interested in the phase or argument of the complex field $V$ let us assume that the oscillator is a dipole rotating in a plane containing the point $P$ of interest. Then the phase of the complex function $V(P, t) \quad i s$ just the angle of rotation of the dipole. If we think of $V$ as a vector from the origin to the point $V$ in the complex plane (where the $\mathrm{X}$-axis is the real axis and the $\mathrm{Y}$-axis is the imaginary axis) then the vector $V$ rotates in synchronism with the physical rotating dipole. classically, as the dipole rotates and radiates it slowly loses energy so the amplitude of the field from a radiating dipole will slowly decay with time. Therefore we can imagine the vector $V$ growing from zero rather quickly and then gradually spiralling in with constant angular velocity until after a long time it vanishes again at the origin. A real field will be a superposition of fields from a great many such oscillators with different frequencies, starting, and sometimes stopping due to collisions, at random times. The resultant complex field vector $V$ will then basically perform a rotation at some mean frequency but will undergo frequent random angular accelerations and decelerations and radial expansions and contractions. If we write $V(t)$ as

$$
V(t)=A(t) \cdot \exp [i \gamma(t)] \cdot \exp \left[-2 \pi i \nu_{0} t\right]
$$

where $\nu_{0}$ is this mean frequency, then the complex quantity $A(t) \cdot \exp [i \gamma(t)] \quad v i e w e d$ as a vector in the complex plane will exhibit the motion of $V(t)$ exclusive of the rotation at

\footnotetext{
${ }^{2}$ See eq. $(10.149)$ on page 49.
} 
frequency $\nu_{0}:$ it will trace a kind of complicated random tangle which contains only the random angular and radial motions of $v(t)$.

In the product $V^{*}\left(P_{1}, t\right) \cdot V\left(P_{2}, t\right)$ the steady rotational motion, exp $\left(2 \pi i \nu_{0} t\right)$, of the complex vectors cancels out so that the cross-spectral density function $\mathrm{W}_{\lambda}\left(\mathrm{P}_{1}, \mathrm{P}_{2}\right)$ is just the average of the product of two randomly dancing vectors like $A(P, t) \cdot \exp [i \gamma(P, t)] \cdot I_{f} P_{1}$ and $\mathrm{P}_{2}$ are very close together then both points will view the source from virtually the same angle and will experience identical fields -- with the possible exception of a small nearly constant phase difference, especially if one point is nearer the source than the other. In this case the complex vectors $A\left(P_{1}, t\right) \cdot \exp \left[i \gamma\left(P_{1}, t\right)\right]$ and $A\left(P_{2}, t\right) \cdot \exp \left[i \gamma\left(P_{2}, t\right)\right]$ will be of equal length and will move in unison maintaining their nearly constant angular phase difference separation $\Delta \gamma=\gamma\left(P_{2}, t\right)-\gamma\left(P_{1}, t\right)$. The cross-spectral density can then be written

$$
\begin{aligned}
\mathrm{w}_{\lambda}\left(\mathrm{P}_{1}, \mathrm{P}_{2}\right) & =\frac{1}{\Delta \lambda} \cdot\left\langle\mathrm{A}^{2}\left(\mathrm{P}_{1}\right)\right\rangle \cdot \exp (\mathrm{i} \Delta \gamma) \\
& =\mathrm{F}_{t, \lambda}\left(\mathrm{P}_{1}\right) \cdot \exp (i \Delta \gamma)
\end{aligned}
$$

or, since $A\left(P_{1}\right)=A\left(P_{2}\right)$, we can write it symmetrically as

$$
w_{\lambda}\left(P_{1}, P_{2}\right)=\sqrt{F_{t, \lambda}\left(P_{1}\right) \cdot F_{t, \lambda}\left(P_{2}\right)} \cdot \exp (i \Delta \gamma)
$$

On the other hand, if the points $P_{1}$ and $P_{2}$ are very far apart it may happen that, due to obstructions in the source, different oscillators contribute to the field at the two points. Or, if one point is much closer to the source than the other, the random dance the vector $A(t) \cdot \exp [i \gamma(t)]$ is performing at $P_{1}$ may be totally dissimilar to that simultaneously occurring at $\mathrm{P}_{2}$ due simply to the finite velocity of light. In such cases the phase $\Delta \gamma\left(\mathrm{P}_{1}, \mathrm{P}_{2}, \mathrm{t}\right)$ of the product

$$
\begin{aligned}
A\left(P_{1}, t\right) & \cdot \exp \left[-i \gamma\left(P_{1}, t\right)\right] \cdot A\left(P_{2}, t\right) \cdot \exp \left[i \gamma\left(P_{2}, t\right)\right] \\
& =A\left(P_{1}, t\right) \cdot A\left(P_{2}, t\right) \cdot \exp \left[i \Delta \gamma\left(P_{1}, P_{2}, t\right)\right]
\end{aligned}
$$

is a completely random angle so that the product complex vector is continuously pointing in different random directions. The average value of such a randomly pointing vector is zero and we have

$$
\mathrm{W}_{\lambda}\left(\mathrm{P}_{1}, \mathrm{P}_{2}\right)=0
$$

These represent the two extreme values for the cross-spectral density function. Usually it lies between these values and we may write

$$
\mathrm{W}_{\lambda}\left(\mathrm{P}_{1}, \mathrm{P}_{2}\right)=\sqrt{\mathrm{F}_{\mathrm{t}, \lambda}\left(\mathrm{P}_{1}\right) \cdot \mathrm{F}_{\mathrm{t}, \lambda}\left(\mathrm{P}_{2}\right)} \cdot \mu\left(\mathrm{P}_{1}, \mathrm{P}_{2}\right)
$$

where

$$
\mu\left(P_{1}, P_{2}\right)=\frac{\left\langle V^{*}\left(P_{1}\right) \cdot V\left(P_{2}\right)\right\rangle}{\Delta \lambda \cdot \sqrt{F_{t, \lambda}\left(P_{1}\right) \cdot F_{t, \lambda}\left(P_{2}\right)}}
$$


is called the complex degree of coherence at wavelength $\lambda$ and at points $\mathrm{P}_{1}$ and $\mathrm{P}_{2} \cdot$ When $\left|\mu\left(\mathrm{P}_{1}, \mathrm{P}_{2}\right)\right|=0$ the field is said to be incoherent at points $\mathrm{P}_{1}$ and $\mathrm{P}_{2} \cdot \mathrm{This}$ implies that knowledge of the phase of the field at either point provides no useful information about the phase at the other point. When $\left|\mu\left(P_{1}, P_{2}\right)\right|=1$ the field is coherent at these two points, and knowledge of the phase of the field at either point permits the phase at the other point to be predicted exactly.

When the field is so incoherent that the only point pairs for which $W_{\lambda}\left(P_{1}, P_{2}\right)$ does not vanish are those within a very short distance of one another (a wavelength or two) -- much closer than any other distance of physical interest such as slit widths -- then it is common to refer to the field as completely incoherent and to write as an approximation ${ }^{1}$

$$
W_{\lambda}\left(P_{1}, P_{2}\right)=\lambda^{2} \cdot A_{0}^{2}\left(P_{1}\right) \cdot \delta\left(x_{1}-x_{2}\right) \cdot \delta\left(y_{1}-y_{2}\right)
$$

where $P_{1}$ and $P_{2}$ are both taken to be in the $X-Y$ plane with coordinates $x_{1}, Y_{1}$ and $x_{2} \cdot y_{2} \cdot{ }^{2}$ Equation $(10.28)$ is assumed to apply at the surface of an incandescent solid, for example.

As another example of the cross-spectral density function let us consider a laser beam of Gaussian cross-section incident on the $X-Y$ plane from a direction in the $X-Z$ plane making an angle of $\theta_{0}$ with the z-axis. The essential geometry is shown in figure 10.2 . In the plane $z=0$ the field will be given as a function of $x$ and $y$ by:

$$
\begin{gathered}
\mathrm{V}(\mathrm{x}, \mathrm{y})=\mathrm{A} \cdot \exp \left(-\frac{\mathrm{y}^{2}+\mathrm{x}^{2} \cdot \cos ^{2} \theta_{0}}{4 \mathrm{~d}^{2}}\right) \cdot \exp \left(\frac{2 \pi i x \cdot \sin \theta_{0}}{\lambda}\right) \\
\cdot \exp (i \gamma) \cdot \exp (-2 \pi i v t),
\end{gathered}
$$

\footnotetext{
${ }^{1}$ Any dependence of the cross-spectral density function or any other radiometric quantity upon position -- $x$, say -- can be construed as being caused by the interference of plane waves propagating with a component of their motion in the $x$ direction. If the cross-spectral density function changes significantly in a distance $l$ then there must be plane wave components present in the beam with wavelengths $\lambda<\ell$ in order to produce such spatial structure. The use of a delta function in the description of incoherent light is not to be taken as suggesting that wavelengths approaching zero are present but rather as a convenient mathematical approximation valid if the dimensions $\ell$ of features of interest are very large compared to the wavelength of light being observed.
}

${ }^{2} \delta\left(x_{1}-x_{2}\right)$ is the Dirac delta function [10.11]. It has the properties:

$$
\delta\left(\mathrm{x}_{1}-\mathrm{x}_{2}\right)=0 \text { for } \mathrm{x}_{1} \neq \mathrm{x}_{2}
$$

and

$$
\int_{x_{1}-a}^{x_{1}+a} \delta\left(x_{1}-x_{2}\right) \cdot d x_{2}=1 \text { for } a n y \quad a>0
$$

Since $\delta(0)=\infty$ we see that eq. (10.28) requires that $F_{t_{,} \lambda}(P)=W_{\lambda}(P, P)=\infty$, an obviously unrealistic feature of this approximation. We should try to think of $\lambda \cdot \delta\left(x_{1}-x_{2}\right)$ and $\lambda \cdot \delta\left(y_{1}-y_{2}\right)$ as convenient mathematical approximations for sharply peaked functions, of width about $\lambda$ and height about 1 . Then we can make the approximate indentifications:

$$
F_{t, \lambda}(P) \sim A_{0}^{2}(P) \quad \text { and } \mu\left(P_{1}, P_{2}\right) \sim \lambda^{2} \cdot \delta\left(x_{1}-x_{2}\right) \cdot \delta\left(y_{1}-y_{2}\right)
$$




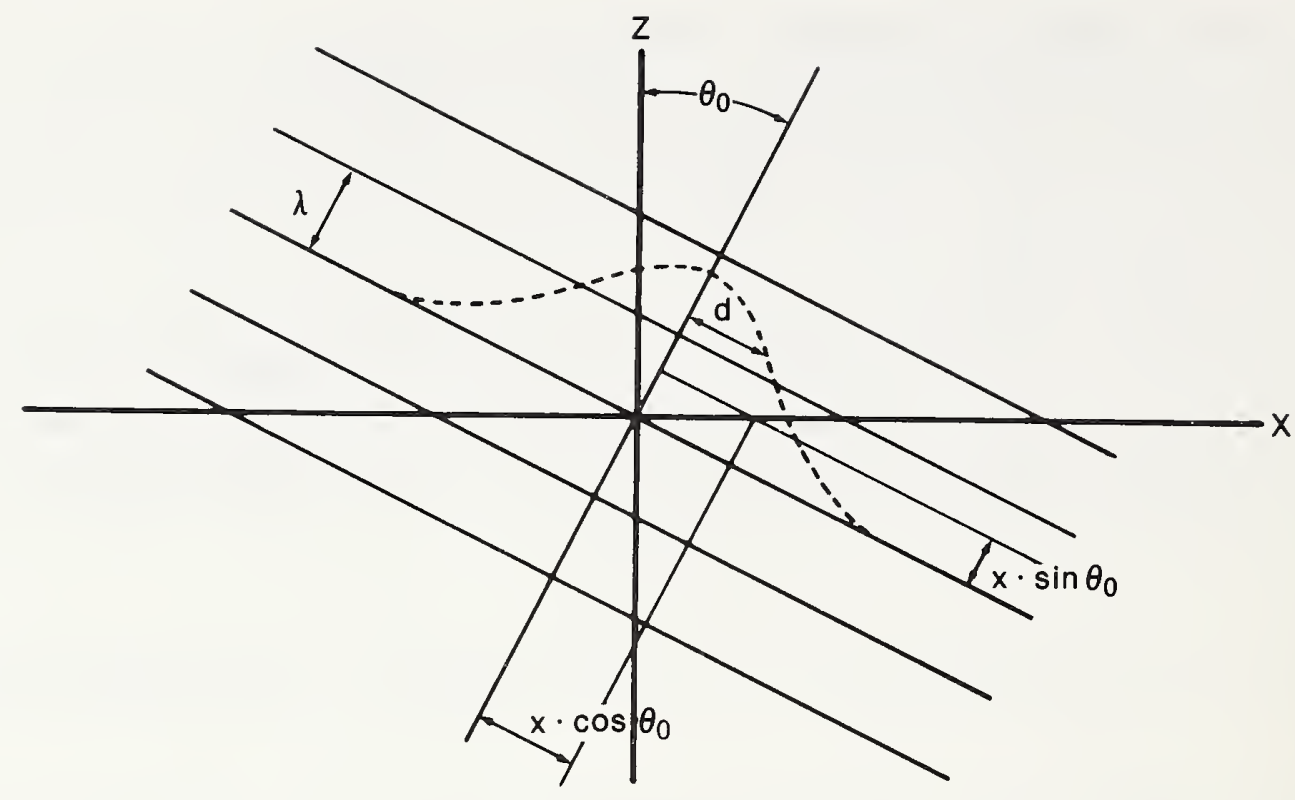

Figure 10.2. A train of plane waves incident on the $X-Y$ plane at an angle $\theta_{0}$ from the $\mathrm{Z}$-axis in the $\mathrm{X}-\mathrm{Z}$ plane and with a Gaussian amplitude distribution.

where $d$ is a measure of the diameter of the beam and $\gamma$ is the phase of the wave at the origin at time zero. The quantity $\frac{2 \pi x \cdot \sin \theta}{\lambda}$ o is the phase difference between a point $x, y$ and the origin expressed in radians. Assuming that the laser is monochromatic we obtain from eq. $(10.14)$

$$
\begin{aligned}
\mathrm{w}_{\lambda}\left(\mathrm{x}_{1}, \mathrm{y}_{1} ; \mathrm{x}_{2}, \mathrm{y}_{2}\right)= & \mathrm{A}_{0}^{2} \cdot \exp \left[-\frac{\mathrm{y}_{1}^{2}+\mathrm{y}_{2}^{2}+\left(\mathrm{x}_{1}^{2}+\mathrm{x}_{2}^{2}\right) \cdot \cos ^{2} \theta_{0}}{4 \mathrm{~d}^{2}}\right] \\
\cdot \exp \left[\frac{2 \pi i \cdot\left(\mathrm{x}_{1}-\mathrm{x}_{2}\right) \cdot \sin \theta_{0}}{\lambda}\right] & \\
= & \exp \left[\frac{2 \pi i \cdot\left(\mathrm{x}_{1}-\mathrm{x}_{2}\right) \cdot \sin \theta_{0}}{\lambda}\right] \cdot \sqrt{\mathrm{F}_{\mathrm{t}, \lambda}\left(\mathrm{x}_{1}, \mathrm{y}_{1}\right) \cdot \mathrm{F}_{\mathrm{t}, \lambda}\left(\mathrm{x}_{2}, \mathrm{y}_{2}\right)},
\end{aligned}
$$

where $A_{0}=A / \sqrt{\Delta \lambda}$.

By comparing eqs. (10.32) and (10.26) it can be seen that the complex degree of coherence is

$$
\mu=\exp \left[\frac{2 \pi i \cdot\left(x_{i}-x_{2}\right) \cdot \sin \theta_{0}}{\lambda}\right]
$$

and again $|\mu|=1$. In this example the spectral fluence rate across the Gaussian beam cross-section is given by 


$$
F_{t, \lambda}(x, y)=A_{0}^{2} \cdot \exp \left(-\frac{y^{2}+x^{2} \cdot \cos ^{2} \theta_{0}}{2 d^{2}}\right)\left[w \cdot m^{-2}\right]
$$

and the phase difference is

$$
\Delta \gamma\left(x_{1}, y_{1} ; x_{2}, y_{2}\right)=\frac{2 \pi \cdot\left(x_{1}-x_{2}\right) \cdot \sin \theta}{\lambda}[\text { rad }] .
$$

The PROPAGATION of CROSS-SPECTRAL DENSITY. If the cross-spectral density function is known at all points in some cross-section of a beam it can be calculated for any other cross-section. The law of propagation of cross-spectral density is

$$
\mathrm{w}_{\lambda}\left(\mathrm{P}_{1}^{\prime}, \mathrm{P}_{2}^{\prime}\right)=\iint_{\text {source }} \mathrm{w}_{\lambda}\left(\mathrm{P}_{1}, \mathrm{P}_{2}\right) \cdot \frac{\exp \left[2 \pi \mathrm{i} \cdot\left(\mathrm{s}_{1}-\mathrm{s}_{2}\right) / \lambda\right] \cdot \cos \theta_{1} \cdot \cos \theta_{2}}{\mathrm{~s}_{1} \cdot \mathrm{s}_{2} \cdot \lambda^{2}} \cdot \mathrm{dP}_{1} \cdot \mathrm{dP}_{2} \cdot
$$

In this equation $\mathrm{W}_{\lambda}\left(\mathrm{P}_{1}, \mathrm{P}_{2}\right)$ is assumed known for all pairs of points $\mathrm{P}_{1}$ and $\mathrm{P}_{2}$ in one cross-section of the beam and $\mathrm{s}_{1}$ and $\mathrm{s}_{2}$ are the optical path lengths $\overline{\mathrm{P}_{1} \mathrm{P}_{1}}$ and $\overline{\mathrm{P}_{2} \mathrm{P}_{2}^{\prime}}$ to the points $\mathrm{P}_{1}^{\prime}$ and $\mathrm{P}_{2}^{\prime}$ in a second cross-section where the cross-spectral density is wanted (see figure 10.3). We will treat these two cross-sections of the beam for convenience as a source plane and a detector plane although, of course, there need not be a physical source or detector in either place. The angles $\theta_{1}$ and $\theta_{2}$ are the angles between the normal to the source plane and the directions to points $\mathrm{P}_{1}^{\prime}$ and $\mathrm{P}_{2}^{\prime}$ from $\mathrm{P}_{1}$ and $\mathrm{P}_{2}$,

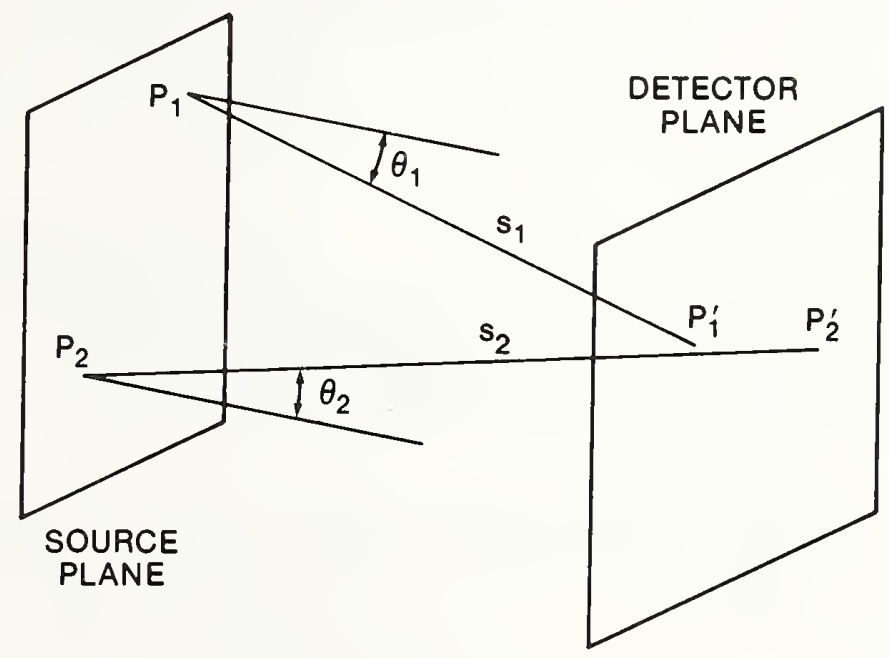

Figure 10.3. The propagation of cross-spectral density $\mathrm{W}_{\lambda}\left(\mathrm{P}_{1}, \mathrm{P}_{2}\right)$, assumed known for all pairs of points $P_{1}$ and $\mathrm{P}_{2}$ in one cross section of a beam (source plane). Optical path lengths $\mathbf{s}_{1}$ and $\mathbf{s}_{2}$ extend to corresponding points $\mathrm{P}_{1}$ and $\mathrm{P}_{2}^{\prime}$ in a second plane where the cross-spectral density is wanted (detector plane). 
respectively. In the integrations, $P_{1}$ and $P_{2}$ each cover the entire source plane independently; that is

$$
\iint_{\text {source }} \mathrm{dP}_{1} \cdot \mathrm{dP}_{2} \equiv \iint_{-\infty}^{\infty} \iint_{-\infty}^{\infty} d \mathrm{x}_{1} \cdot d \mathrm{y}_{1} \cdot d \mathrm{x}_{2} \cdot d \mathrm{y}_{2}
$$

where $x_{1}, y_{1}$ and $x_{2}, y_{2}$ are the coordinates of $P_{1}$ and $P_{2}$ in the source plane.

The factors appearing in eq. (10.36) are easily understood. The exponential is just the phase change resulting from the difference in the path lengths $\overline{\mathrm{P}_{1} \mathrm{P}_{1}}$ and $\overline{\mathrm{P}_{2} \mathrm{P}_{2}} \cdot$ This difference is divided by $\lambda$ to obtain the number of periods and then multiplied by $2 \pi$ to express the phase difference in radians. The cosine factors express the reduction in projected size of the elements of area $\mathrm{dP}=\mathrm{dx} \cdot \mathrm{dy}$ when viewed from the direction of $\mathrm{P}^{\prime}$. The distances $s_{1}$ and $s_{2}$ in the denominator are in effect a statement of the inverse square law. The factor $\lambda^{2}$ in the denominator is required for dimensional homogeneity and to assure the correct propagation of fluence rate computed from eq. (10.36) in free space. Since, by Huygens principle, all points $P$ can contribute to each point $P^{\prime}$, the integration covers the entire source plane for both $P_{1}$ and $P_{2}$. Equation (10.36) is valid for source dimensions and distances $\mathbf{s}_{1}$ and $\mathbf{s}_{2}$. which are large compared to the wavelength of 1 ight.

It is in the evaluation of the integrals of eq. (10.36) that the difficulties of the treatment of coherence occur. To illustrate the kinds of approximations which are employed to render these integrals tractable and to better illustrate the meanings of the factors appearing in the integrals let us evaluate eq. (10.36) for a simple situation. In figure 10.4 we show a point $P$ in a source plane where the cross-spectral density is assumed known and a typical field point $\mathrm{P}^{\prime}$ where the cross-spectral density is desired. The 'source' plane need not be a real source, of course. It can be any plane through a beam anywere. We take the source plane to be the $\mathrm{X}-\mathrm{Y}$ plane and the origin to lie in this plane near the center of the beam.

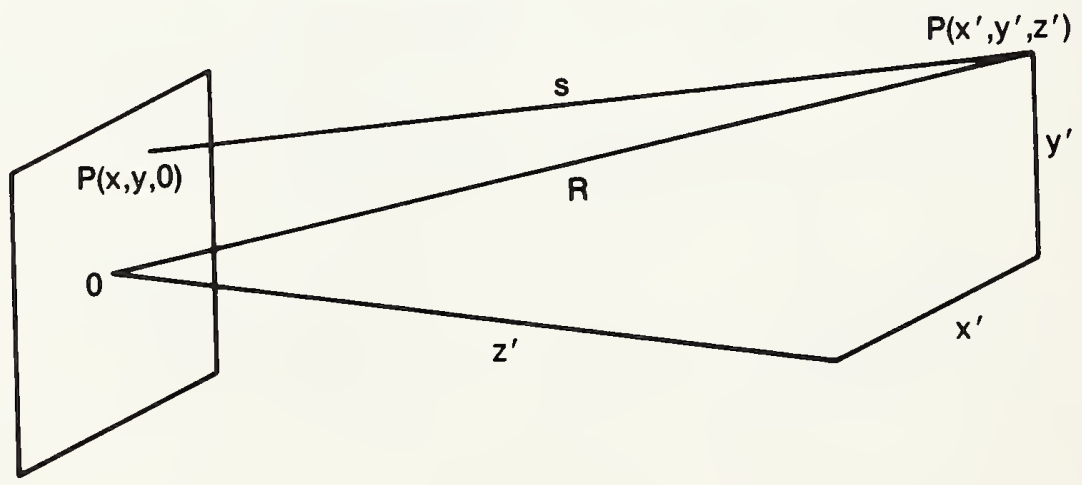

Figure 10.4. A typical field point $P^{\prime}\left(x^{\prime}, y^{\prime}, z^{\prime}\right)$ is shown at a distance $R$ from the origin and a distance $s$ from a point $P(x, y, 0)$ in the source plane ( $X-Y$ plane). 
The first task is to express the distance $s$ from $P$ to $P^{\prime}$ in terms of the variables of integration -- the coordinates $x$ and $y$ of $P$. This is simply

$$
\begin{aligned}
s & =\sqrt{\left(x^{\prime}-x\right)^{2}+\left(y^{\prime}-y\right)^{2}+z^{\prime 2}} \\
& =\sqrt{R^{2}-2 x^{\prime} x-2 y^{\prime} y+x^{2}+y^{2}} \\
& =R \cdot \sqrt{1-2\left(x^{\prime} x+y^{\prime} y\right) / R^{2}+\left(x^{2}+y^{2}\right) / R^{2}}
\end{aligned}
$$

where $\mathrm{R}^{2}=\mathrm{x}^{\prime 2}+\mathrm{y}^{\prime 2}+\mathrm{z}^{\prime 2}$ and the primed coordinates are the coordinates of $\mathrm{P}^{\prime}$. We now assume that the source dimensions are small compared to $R$. That is $x / R<1$ and $y / R \ll 1$. Then we can expand the square root in eq. (10.38) and discard high order terms to get:

$$
s \approx R-\left[\left(x^{\prime} x+y^{\prime} y\right) / R\right]+\frac{1}{2}\left(x^{2}+y^{2}\right) / R
$$

Next we note that only a small error is introduced by replacing $s_{1}$ and $s_{2} \quad$ by $R_{1}$ and $R_{2}$ in the denominator of the integral. A similar substitution in the argument of the exponential is not permissible because this function changes quite rapidly with small changes in $s_{1}{ }^{-s_{2}}{ }$ This is easily seen by writing

$$
\exp \left[2 \pi i \cdot\left(s_{1}-s_{2}\right) / \lambda\right]=\cos \left[2 \pi \cdot\left(s_{1}-s_{2}\right) / \lambda\right]+i \cdot \sin \left[2 \pi \cdot\left(s_{1}-s_{2}\right) / \lambda\right]
$$

A change of $\mathbf{s}_{1} \mathbf{s}_{2}$ by as little as one wavelength will drive the trigonometric functions through an entire cycle while the same change affects the product $s_{1} \cdot s_{2}$ in the denominator by only a part in $10^{6}$ (assuming $s_{1}$ and $s_{2}$ are of the order of a meter). So, making these approximations and also approximating $\cos \theta$ by $z^{\prime} / R$, which is $\operatorname{consistent~with~}$ $x, y<R$, we obtain

$$
\begin{aligned}
w_{\lambda}\left(x_{1}^{\prime}, y_{1}^{\prime}, z_{1}^{\prime} ; x_{2}^{\prime}, y_{2}^{\prime}, z_{2}^{\prime}\right)= & \frac{z_{1}^{\prime} z_{2}^{\prime}}{R_{1}^{2} R_{2}^{2} \lambda^{2}} \cdot \exp \left[\frac{2 \pi i}{\lambda}\left(R_{1}-R_{2}\right)\right] \cdot \iiint \int_{\text {source }} w_{\lambda}\left(x_{1}, y_{1} ; x_{2}, y_{2}\right) \\
& \cdot \exp \left[-\frac{2 \pi i}{\lambda} \cdot\left(\frac{x_{1} x_{1}^{\prime}+y_{1} y_{1}^{\prime}}{R_{1}}-\frac{x_{2} x_{2}^{\prime}+y_{2} y_{2}^{\prime}}{R_{2}}\right)\right] \\
& \cdot \exp \left[\frac{\pi i}{\lambda} \cdot\left(\frac{x_{1}^{2}+y_{1}^{2}}{R_{1}}-\frac{x_{2}^{2}+y_{2}^{2}}{R_{2}}\right)\right] \cdot d x_{1} \cdot d_{y_{1}} \cdot d_{x_{2}} \cdot d y_{2} \cdot
\end{aligned}
$$

In this expression the integration limits describe the cross-section of the beam or the area of the source and we recall that we have assumed that this is very small compared to the distance to the points $\mathrm{P}_{i}^{\prime}$ and $\mathrm{P}_{2}^{\prime}$.

The last exponential factor in eq. (10.41) involving terms in $\mathrm{x}_{1}{ }^{2}, \mathrm{y}_{1}{ }^{2}$, etc., identifies this integral as being akin to diffraction integrals in the Fresnel approximation. In coherence calculations this factor is frequently set to unity -- consistent with the stronger assumption that the source dimensions are small compared to $\sqrt{\lambda R}$. With this further simplification eq. (10.41) becomes 


$$
\begin{aligned}
w_{\lambda}\left(x_{1}^{\prime}, y_{1}^{\prime}, z_{1}^{\prime} ; x_{2}^{\prime}, y_{2}^{\prime}, z_{2}^{\prime}\right)= & \frac{z_{1}^{\prime} z_{2}^{\prime}}{R_{1}^{2} R_{2}^{2} \lambda^{2}} \cdot \exp \left[\frac{2 \pi i}{\lambda} \cdot\left(R_{1}-R_{2}\right)\right] \cdot \iiint \int_{\text {source }} w_{\lambda}\left(x_{1}, y_{1} ; x_{2}, y_{2}\right) \\
& \cdot \exp \left[-\frac{2 \pi i}{\lambda}\left(\frac{x_{1} x_{1}^{\prime}+y_{1} y_{1}^{\prime}}{R_{1}}-\frac{x_{2} x_{2}^{\prime}+y_{2} y_{2}^{\prime}}{R_{2}}\right)\right] \cdot d x_{1} \cdot d y_{1} \cdot d x_{2} \cdot d y_{2}
\end{aligned}
$$

which is in the Fraunhofer approximation. This is the expression we will use in all our examples although it should be noted that if $\mathrm{w}_{\lambda}\left(\mathrm{x}_{1}, \mathrm{y}_{1} ; \mathrm{x}_{2}, \mathrm{y}_{2}\right)$ is so complicated that the integrals must be evaluated numerically anyway then consideration should probably be given to including additional terms or to calculating $\left(s_{1}-s_{2}\right)$ exactly using eq. (10.38) wherever it will result in higher accuracy. For the most part we can justify the use of the Fraunhofer approximation even when the source dimensions are not small compared to $\sqrt{\lambda R}$ on the grounds that this will give us a worst-case estimate of coherence and diffraction effects. The true flux distribution.will always lie somewhere between the predictions of geometrical optics theory and predictions in the Fraunhofer approximation in the sense that as the distance $z$ ' to the observing surface increases, sharp edges and shadows predicted by geometrical optics gradually become fringed and fuzzy, evolving, for sufficiently large $z^{\prime}$ or $R$, into the Fraunhofer irradiation pattern. This is a smoothly periodic pattern dependent upon the point of observation only through the angular parameters $x^{\prime} / R$ and $y^{\prime} / R$. If a calculation in this approximation reveals that diffraction will cause no significant problems in a measurement situation then a more exact computation generally is unnecessary. This will frequently be the case if the measurement situation requires only ratios of measurements on similar systems. However, if an accurate estimate of the loss of flux from a geometrical beam is required as, for example, if the cross-sectional area of the beam doesn't cancel out in the simple geometrical ray analysis of the measurements, then it is likely that a more exact calculation [eq. (10.38)] will be necessary. ${ }^{1}$

Equation (10.42) is about as far as eq. (10.36) can be simplified without some knowledge of $W_{\lambda}\left(P_{1}, P_{2}\right)$. Included among the next twenty-five pages or so are some examples in which expressions such as eq. (10.42) are further evaluated in considerable, tedious detail. These lengthy passages are set in a smaller type to permit the reader to identify them and skip over them if he wishes. We present such examples here to show that the propagation law of crossspectral density is not just another pretty theory but that numbers can be put in and useful results obtained. The examples will also indicate how the coherence state can, in certain circumstances, significantly affect the results of a radiometric measurement when high spectral, spatial, or temporal resolution measurements are attempted.

As the first example of the use of eq. (10.42) we will apply it to one of the simplest possible sources -- a source in the plane $z=0$ that is uniform, rectangular, and incoherent with [from eq. (10.28)]

$$
\begin{aligned}
& w_{\lambda}\left(x_{1}, y_{1} ; x_{2}, y_{2}\right)=\lambda^{2} A_{0}^{2} \cdot \delta\left(x_{1}-x_{2}\right) \cdot \delta\left(y_{1}-y_{2}\right)
\end{aligned}
$$

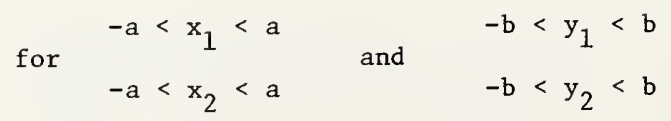

and $\quad \mathrm{w}_{\lambda}\left(\mathrm{x}_{1}, \mathrm{y}_{1} ; \mathrm{x}_{2}, \mathrm{y}_{2}\right)=0$ otherwise.

\footnotetext{
$\overline{{ }^{1} \text { See reference }}[10.12]$ for an example of such calculations.
} 
Then eq. (10.42) will allow us to calculate the cross-spectral density for any pair of points $P_{1}^{\prime}$ (at $\left.x_{1}^{\prime}, y_{1}^{\prime}, z_{1}^{\prime}\right)$ and $P_{2}^{\prime}$ (at $\left.x_{2}^{\prime}, y_{2}^{\prime}, z_{2}^{\prime}\right)$ provided that both $R_{1}{ }^{2}\left(=x_{1}^{\prime 2}+y_{1}^{\prime 2}+z_{1}^{\prime 2}\right)$ and $\mathrm{R}_{2}{ }^{2}\left(=\mathrm{x}_{2}^{\prime 2}+\mathrm{y}_{2}^{\prime 2}+\mathrm{z}_{2}^{\prime 2}\right)$ are much greater than $\mathrm{a}^{2}+\mathrm{b}^{2}$. If we substitute expression (10.43) into eq. (10.42) we can immediately carry out the integrations over $x_{2}$ and $y_{2}$ to give

$$
\begin{aligned}
W_{\lambda}\left(x_{1}^{\prime}, y_{1}^{\prime}, z_{1}^{\prime} ; x_{2}^{\prime}, y_{2}^{\prime}, z_{2}^{\prime}\right)= & \frac{z_{1}^{\prime} z_{2}^{\prime}}{R_{1}^{2} R_{2}^{2}} \cdot A_{0}^{2} \cdot \exp \left[\frac{2 \pi i}{\lambda} \cdot\left(R_{1}-R_{2}\right)\right] \\
& \cdot \int_{-a}^{a} \int_{-b}^{b} \exp \left[-\frac{2 \pi i}{\lambda}\left(\frac{x_{1} \cdot x_{1}^{\prime}+y_{1} \cdot y_{1}^{\prime}}{R_{1}}-\frac{x_{1} x_{2}^{\prime}+y_{1} y_{2}^{\prime}}{R_{2}}\right)\right] \cdot d x_{1} \cdot d y_{1} \cdot
\end{aligned}
$$

The double integral in eq. (10.44) factors into two similar elementary integrals:

$$
\begin{aligned}
\int_{-a}^{a} \int_{-b}^{b}= & \int_{-a}^{a} \exp \left[-\frac{2 \pi i}{\lambda} \cdot\left(\frac{x_{1}^{\prime}}{R_{1}}-\frac{x_{2}^{\prime}}{R_{2}}\right) \cdot x_{1}\right] \cdot d x_{1} \cdot \int_{-b}^{b} \exp \left[-\frac{2 \pi i}{\lambda} \cdot\left(\frac{y_{1}^{\prime}}{R_{1}}-\frac{y_{2}^{\prime}}{R_{2}}\right) \cdot y_{1}\right] \cdot d y_{1} \\
& =2 a \cdot\left\{\frac{\sin \left[\frac{2 \pi a}{\lambda} \cdot\left(\frac{x_{1}^{\prime}}{R_{1}}-\frac{x_{2}^{\prime}}{R_{2}}\right)\right]}{\frac{2 \pi a}{\lambda} \cdot\left(\frac{x_{1}^{\prime}}{R_{1}}-\frac{x_{2}^{\prime}}{R_{2}}\right)}\right\} \cdot 2 b \cdot\left\{\frac{\sin \left[\frac{2 \pi b}{\lambda} \cdot\left(\frac{y_{1}^{\prime}}{R_{1}}-\frac{y_{2}^{\prime}}{R_{2}}\right)\right]}{\frac{2 \pi b}{\lambda} \cdot\left(\frac{y_{1}^{\prime}}{R_{1}}-\frac{y_{2}^{\prime}}{R_{2}}\right)}\right\} .
\end{aligned}
$$

It turns out that these integrals are among the few integrals likely to arise in these problems which can be expressed in a closed form and can be readily visualized. As a result, coherence problems which lead to such integrals are among the few which are ever carried through to completion and so the functional form $\sin (u) / u$ has achieved an importance which is probably richly undeserved. It has a special name

$$
\operatorname{sinc}(u) \equiv \sin (\pi u) /(\pi u) .
$$

(See figure 10.5)

If we employ this notation eq. (10.44) becomes

$$
\begin{aligned}
\mathrm{w}_{\lambda}\left(\mathrm{x}_{1}^{\prime}, \mathrm{y}_{1}^{\prime}, z_{1}^{\prime} ; \mathrm{x}_{2}^{\prime}, \mathrm{y}_{2}^{\prime}, z_{2}^{\prime}\right)= & \frac{z_{1}^{\prime} z_{2}^{\prime}}{\mathrm{R}_{1}^{2} \mathrm{R}_{2}^{2}} \cdot \mathrm{A}_{0}^{2} \cdot \exp \left[\frac{2 \pi i}{\lambda} \cdot\left(\mathrm{R}_{1}-\mathrm{R}_{2}\right)\right] \\
& \cdot 4 \mathrm{ab} \cdot \operatorname{sinc}\left[\frac{2 \mathrm{a}}{\lambda} \cdot\left(\frac{\mathrm{x}_{1}^{\prime}}{\mathrm{R}_{1}}-\frac{\mathrm{x}_{2}^{\prime}}{\mathrm{R}_{2}}\right)\right] \cdot \operatorname{sinc}\left[\frac{2 b}{\lambda} \cdot\left(\frac{y_{1}^{\prime}}{\mathrm{R}_{1}}-\frac{y_{2}^{\prime}}{\mathrm{R}_{2}}\right)\right] .
\end{aligned}
$$

Let us first consider the case where both points $P_{1}^{\prime}$ and $P_{2}^{\prime}$ are equally far from the source so that $R_{1}=R_{2}=R$. Then eq. (10.46) simplifies a little to give

$$
\begin{aligned}
w_{\lambda}\left(x_{1}^{\prime}, y_{1}^{\prime}, z_{1}^{\prime} ; x_{2}^{\prime}, y_{2}^{\prime}, z_{2}^{\prime}\right)= & 4 a b \cdot A_{0}^{2} \cdot \frac{z_{1}^{\prime} z_{2}^{\prime}}{R^{4}} \\
& \cdot \operatorname{sinc}\left[\frac{2 a\left(x_{1}^{\prime}-x_{2}^{\prime}\right)}{\lambda R}\right] \cdot \operatorname{sinc}\left[\frac{2 b\left(y_{1}^{\prime}-y_{2}^{\prime}\right)}{\lambda R}\right] .
\end{aligned}
$$




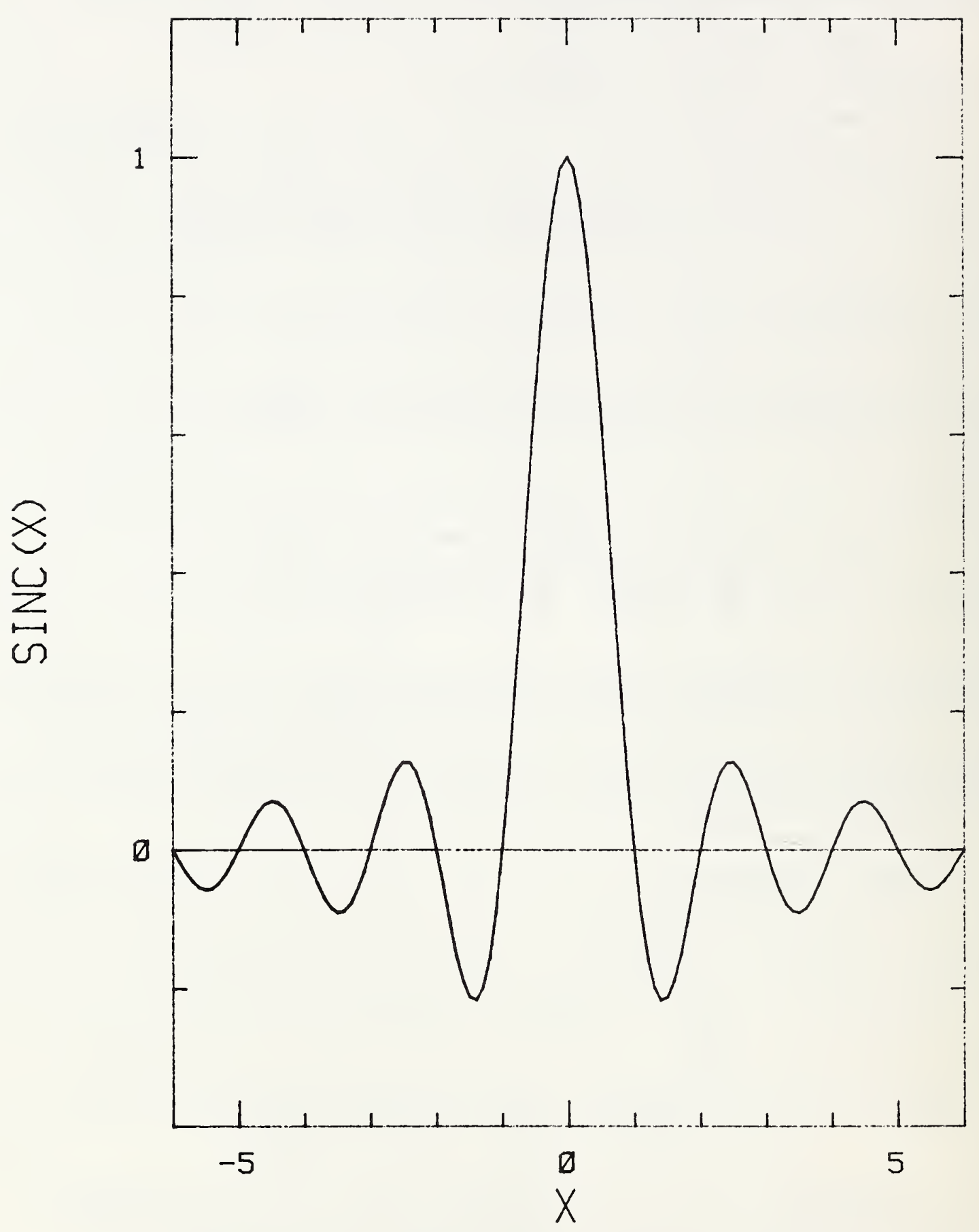

Figure 10.5. The sinc function: $\operatorname{sinc} x \equiv \sin (\pi x) /(\pi x)$. 
There are several interesting points to observe about this cross-spectral density function. First, the radiation at a distance from the source is no longer incoherent -- there are no delta functions in eq. (10.47) The radiation has become partially coherent merely by the fact of propagation. For a very large source dimension, a, the sinc function will oscillate and decrease rapidly as $x_{1}^{\prime}-x_{2}^{\prime}$ increases (see figure 10.5) so that eq. (10.47) effectively approaches delta function behavior ${ }^{1}$ for very large incoherent sources. A second feature of eq. (10.47) is that the $y$-dependence is totally independent of the $x$-dependence in that, if the $y$-dimension of the source is changed, only the y-scale of the cross-spectral density function will be changed. This is a characteristic of solutions in the Fraunhofer approximation of problems involving rectangular sources or apertures: provided $\mathrm{W}_{\lambda}\left(\mathrm{P}_{1}, \mathrm{P}_{2}\right)$ has this property the solution factors into independent double integrals -- one double integral involving only $x_{1}$ and $x_{2}$, the other double integral involving only $y_{1}$ and $y_{2}$. Another interesting feature of eq. (10.47) is that the $x$-dependent factor depends only upon $x_{1}^{\prime}$ and $x_{2}^{\prime}$ through their difference $x_{1}^{\prime}-x_{2}^{\prime}$, and similarly the $y-$ dependent factor depends only upon the difference $y_{1}^{\prime}-y_{2}^{\prime}$. When a function depends only upon differences in this way it is said to be stationary because it looks the same in the neighborhood of one $x^{\prime}, y^{\prime}$ point as it does in the neighborhood of any other point. In many coherence problems where the cross-spectral density function is not known the property of stationarity is assumed in the interests of simplicity and we see that this property can arise naturally and is therefore a reasonable assumption. Finally, we can calculate the fluence rate from eq. (10.47) by setting $\mathrm{P}_{1}^{\prime}=\mathrm{P}_{2}^{\prime}:$

$$
F_{t, \lambda}\left(P^{\prime}\right)=w_{\lambda}\left(x^{\prime}, y^{\prime}, z^{\prime} ; x^{\prime}, y^{\prime}, z^{\prime}\right)=4 a b \cdot A_{0}^{2} \cdot \frac{\cos ^{2} \theta}{R^{2}}
$$

where $\theta$ is the angle between the normal to the surface and the viewing direction. We see that, in contrast to expressions we shall derive below, there are no diffraction effects here: the fluence rate at $P^{\prime}$ depends upon the location of $P^{\prime}$ only through the inverse square of the distance from the source and through the simple angular factor $\cos ^{2} \theta$. The reason, incidentally, that this latter factor in this equation and in eq. (10.51) below is $\cos ^{2} \theta$ and not $\cos \theta$ is that this source is not lambertian. Such a completely incoherent source as this may not even be physically realizable; however, it is a useful limiting case which is comparatively easily treated and is sufficiently realistic for many purposes. We will briefly treat a lambertian source later [see, e.g., eqs. (10.166a) to (10.171a)]. From eq. (10.50) we can calculate the radiant intensity of the source [eq. (10.16)] as

${ }^{1}$ One representation of the Dirac delta function is

$$
\delta(x)=\operatorname{Lim}_{a \rightarrow \infty}\left(\frac{\sin a x}{\pi x}\right)
$$

In the limit as $a$ and $b$ become infinite the cross-spectral density according to eq. (10.47) approaches

$$
\lambda^{2} \cdot A_{0}^{2} \cdot\left(\frac{\pi \cdot z_{1}^{\prime}}{R}\right)^{2} \cdot \delta\left(x_{1}^{\prime}-x_{2}^{\prime}\right) \cdot \delta\left(y_{1}^{\prime}-y_{2}^{\prime}\right)
$$

which, without the factor $\left(\frac{\pi \cdot z_{1}^{\prime}}{\mathrm{R}}\right)^{2}$, would be exactly correct [compare eq. (10.28)]. The approximations leading to eq. (10.41) and (10.42) are of course, totally invalid in this limit and evidently the erroneous factor is a consequence of this inconsistency. 


$$
I_{\lambda}(\theta)=4 \mathrm{ab} \cdot \mathrm{A}_{0}^{2} \cdot \cos ^{2} \theta
$$

From eqs. $(10.47),(10.50)$, and $(10.26)$ we obtain

$$
\mu\left(P_{1}^{\prime}, P_{2}^{\prime}\right)=\operatorname{sinc}\left[\frac{2 a \cdot\left(x_{1}^{\prime}-x_{2}^{\prime}\right)}{\lambda R}\right] \cdot \operatorname{sinc}\left[\frac{2 b \cdot\left(y_{1}^{\prime}-y_{2}^{\prime}\right)}{\lambda R}\right]
$$

for the degree of coherence. The dependence of the degree of coherence upon the separation of the points in the observing surface is essentially that given in figure 10.5. The degree of coherence is 1 for points very close together and approaches zero for points very far apart. Negative values for the degree of coherence for certain point separations mean that the fields at such a pair of points are predicted to be out of phase. A small value for $\left|\mu\left(P_{1}^{\prime}, P_{2}^{\prime}\right)\right|$ means that the phase difference between points $\mathrm{P}_{1}^{\prime}$ and $\mathrm{P}_{2}^{\prime}$ is not highly predictable: an element of randomess is present.

In general the degree of coherence (like the cross-spectral density function) is defined in terms of the coordinates of pairs of points so that even for a fixed frequency or wavelength it would require a 7-dimensional space to plot the absolute value of this function. In order to extract some measure of coherence which is easier to grapple with, the terms coherence length, coherence time, and coherence area are often used. These are all rough measures of the separation between the two points $\mathrm{P}_{1}$ and $\mathrm{P}_{2}$ at which the degree of coherence is reduced to some arbitrary level ${ }^{1}$ such as $\frac{1}{2}$ or $1 / e^{--}$the exact level is not important as the terms are only semi-quantitative at best. Coherence length refers to a distance in the direction of propagation -- our z-axis -- and could, for example, be defined in the neighborhood of any axial point $(0,0, z)$ as that value, $\Delta z_{c}$, for which

$$
\left|\mu\left(0,0, z+\frac{1}{2} \Delta z_{c} ; 0,0, z-\frac{1}{2} \Delta_{c}\right)\right|=\frac{1}{2} \text {. }
$$

The coherence time is the time required for light to travel the distance $\Delta \mathbf{z}_{c}$ :

$$
\Delta t_{c}=\Delta z_{c} / c
$$

where $c$ is the velocity of light. Of more interest in most radiometric situations is the measure of the coherence distance, known as the spatial coherence interval, in directions perpendicular to the direction of propagation. These coherence intervals, $\Delta x_{C}$ and $\Delta y_{C}$, could be defined by

and

$$
\left|\mu\left(x+\frac{1}{2} \Delta x_{C}, y, z ; x-\frac{1}{2} \Delta x_{C}, y, z\right)\right|=\frac{1}{2}
$$

$$
\left|\mu\left(x, y+\frac{1}{2} \Delta y_{C}, z ; x, y-\frac{1}{2} \Delta y_{C}, z\right)\right|=\frac{1}{2}
$$

The area $\Delta \mathrm{A}_{\mathrm{C}}=\Delta \mathrm{x}_{\mathrm{C}} \cdot \Delta \mathrm{y}_{\mathrm{C}}$ known as the coherence area is then the region about the point $(x, y, z)$ in the plane normal to the direction of propagation within which a high degree of coherence exists; the field outside this area will be only weakly correlated with the field at $(x, y, z)$.

We see from figure 10.5 and eq. (10.52) that the field from a rectangular incoherent source exhibits a coherence interval in the $x$-direction determined by

${ }^{1}$ In the case of functions, such as sinc, which take on negative values, the first zero of the function is often used. 


$$
\frac{2 \mathrm{a} \cdot\left(\frac{1}{2} \Delta \mathrm{x}_{\mathrm{c}}\right)}{\lambda \mathrm{R}}=0.6 \text { or } \Delta \mathrm{x}_{\mathrm{C}}=\frac{0.6 \lambda \mathrm{R}}{\mathrm{a}} \text {, }
$$

where 0.6 is approximately the value of $u$ which satisfies $\operatorname{sinc}(u)=\frac{1}{2}$. The coherence interval in the $y$-direction is the same expression with $b$ replacing a. The coherence area is thus

$$
\Delta \mathrm{A}_{\mathrm{c}}=\frac{0.36 \lambda^{2} \mathrm{R}^{2}}{\mathrm{ab}} \approx \lambda^{2} / \Delta \omega
$$

where $\Delta \omega\left(=4 \mathrm{ab} / \mathrm{R}^{2}\right)$ is the solid angle subtended by the source from the observation point $(x, y, z)$. In this case the values for the coherence interval and coherence area are independent of $x$ and $y$ because $\mu$ is stationary. Equation (10.57) says that for any source, the irradiation in a distant plane will be highly coherent over areas at least as large as $\lambda^{2} / \Delta \omega$. If the source is partially coherent the areas of coherence will be larger. If an aperture of this size is placed in the field from the source the radiation passing through it will be highly polarized and will exhibit strong diffraction effects.

Returning now to eq. (10.46), which is the general expression for the cross-spectral density due to a distant, uniform, rectangular, incoherent source, where $R_{1}$ is not necessarily the same as $R_{2}$, we find the fluence rate still given by eq. (10.50) but the degree of coherence is

$$
\mu\left(P_{1}^{\prime}, P_{2}^{\prime}\right)=\operatorname{sinc}\left[\frac{2 a}{\lambda} \cdot\left(\frac{x_{1}^{\prime}}{R_{1}}-\frac{x_{2}^{\prime}}{R_{2}}\right)\right] \cdot \operatorname{sinc}\left[\frac{2 b}{\lambda} \cdot\left(\frac{y_{1}^{\prime}}{R_{1}}-\frac{y_{2}^{\prime}}{R_{2}}\right)\right] .
$$

This is still a solution in the Fraunhofer approximation [eq. (10.42)]. In this approximation the spatial structure of the field, aside from the inverse square dependence, is a function only of the angular position of the observation point. This is evident in eqs. (10.50) and (10.58) in the dependence upon $P_{1}^{\prime}$ and $P_{2}^{\prime}$ only through the ratios $x_{1}^{\prime} / R_{1}, y_{1}^{\prime} / R_{1}, z_{1}^{\prime} / R_{1}$, etc. For this reason the field at two points $P_{1}^{\prime}$ and $P_{2}^{\prime}$ which lie in the same direction from the source (so that $x_{1}^{\prime} / R_{1}=x_{2}^{\prime} / R_{2}$ and $\left.y_{1}^{\prime} / R_{1}=y_{2}^{\prime} / R_{2}\right)$ will be calculated to be coherent $(|\mu|=1)$ in this approximation. It is unlikely in radiometry that a higher approximation would be needed but, if it were, then the Fresnel approximation of eq. (10.41) is the next step to try if a closed-form analytic solution is the goal. For the present example of a uniform, rectangular, incoherent source eq. (10.41) can be evaluated exactly in terms of Fresnel integrals. Aside from showing that the degree of coherence does, after all, depend upon the difference in radial distances when these distances are too small to justify the Fraunhofer approximation, the solution is of little interest. It is too complicated to allow any easy intuitive interpretation and, moreover, since real sources are never perfectly uniform, rectangular, and incoherent the accuracy enhancement obtained by employing a better but still incomplete analytical approximation would seem to be of marginal practical value.

Another example of the propagation of cross-spectral density that is easy to evaluate is obtained by replacing the incoherent source we just treated by a coherent one. This could, for example, be a small rectangular aperture of dimensions $2 a$ by $2 b$ irradiated by a laser. We will assume that the angle of incidence of the laser beam is $\theta_{0}$ and take the plane of incidence to be the $\mathrm{X}-\mathrm{Z}$ plane. We will also assume that the amplitude is uniform over the aperture so the cross-spectral density at the aperture has the phase dependence of eq. (10.32) but with a stepfunction replacing the Gaussian: 


$$
\begin{aligned}
& \mathrm{W}_{\lambda}\left(\mathrm{x}_{1}, \mathrm{y}_{1} ; \mathrm{x}_{2}, \mathrm{y}_{2}\right)=\mathrm{A}_{0}{ }^{2} \cdot \exp \left[\frac{2 \pi i}{\lambda} \cdot\left(\mathrm{x}_{1}-\mathrm{x}_{2}\right) \cdot \sin \theta_{0}\right] \quad-a<\mathrm{x}_{1}<\mathrm{a} \\
& \text { for } \quad-a<x_{2}<a \\
& -b<y_{1}<b \\
& -\mathrm{b}<\mathrm{y}_{2}<\mathrm{b}
\end{aligned}
$$

$=0$ otherwise.

When we insert eq. (10.59) into eq. (10.42) we obtain

$$
\begin{aligned}
& \mathrm{w}_{\lambda}\left(\mathrm{x}_{1}^{\prime}, \mathrm{y}_{1}^{\prime}, \mathrm{z}_{1}^{\prime} ; \mathrm{x}_{2}^{\prime}, \mathrm{y}_{2}^{\prime}, \mathrm{z}_{2}^{\prime}\right)= \frac{\mathrm{z}_{1}^{\prime} \mathrm{z}_{2}^{\prime}}{\mathrm{R}_{1}^{2} \mathrm{R}_{2}^{2} \lambda^{2}} \cdot \exp \left[\frac{2 \pi i}{\lambda} \cdot\left(\mathrm{R}_{1}-\mathrm{R}_{2}\right)\right] \\
& \cdot \int_{-\mathrm{a}}^{\mathrm{a}} \int_{-\mathrm{b}}^{\mathrm{b}} \int_{-\mathrm{a}}^{\mathrm{a}} \int_{-b}^{b} \mathrm{~A}_{\mathrm{o}}^{2} \cdot \exp \left[\frac{2 \pi i}{\lambda} \cdot\left(\mathrm{x}_{1}-\mathrm{x}_{2}\right) \cdot \sin \theta_{0}\right] \\
& \cdot \exp \left[-\frac{2 \pi i}{\lambda} \cdot\left(\frac{\mathrm{x}_{1} \mathrm{x}_{1}^{\prime}+\mathrm{y}_{1} \mathrm{y}_{1}^{\prime}}{\mathrm{R}_{1}}-\frac{\mathrm{x}_{2} \mathrm{x}_{2}^{\prime}+\mathrm{y}_{2} \mathrm{y}_{2}^{\prime}}{\mathrm{R}_{2}}\right)\right] \cdot d \mathrm{dx}_{1} \cdot \mathrm{dy}_{1} \cdot \mathrm{dx}_{2} \cdot \mathrm{dy}_{2} \cdot
\end{aligned}
$$

This multiple integral can be factored into a factor depending only upon the coordinates of $P_{1}$ and a factor depending only upon the coordinates of $P_{2}$, and since the variables of integration are only dummy variables, the form of one of these factors is just the complex conjugate of the form of the other:

$$
\mathrm{w}_{\lambda}\left(\mathrm{x}_{1}^{\prime}, \mathrm{y}_{1}^{\prime}, \mathrm{z}_{1}^{\prime} ; \mathrm{x}_{2}^{\prime}, \mathrm{y}_{2}^{\prime}, \mathrm{z}_{2}^{\prime}\right)=\mathrm{v}_{\lambda}^{*}\left(\mathrm{x}_{1}^{\prime}, \mathrm{y}_{1}^{\prime}, \mathrm{z}_{1}^{\prime}\right) \cdot \mathrm{v}_{\lambda}\left(\mathrm{x}_{2}^{\prime}, \mathrm{y}_{2}^{\prime}, \mathrm{z}_{2}^{\prime}\right)
$$

where

$$
\begin{gathered}
v_{\lambda}\left(x^{\prime}, y^{\prime}, z^{\prime}\right)=\frac{z^{\prime}}{R^{2} \lambda} \cdot \exp \left(\frac{2 \pi i R}{\lambda}\right) \cdot \int_{-a}^{a} \int_{-b}^{b} A_{0} \cdot \exp \left(\frac{2 \pi i}{\lambda} \cdot x \cdot \sin \theta_{0}\right) \\
\cdot \exp \left[-\frac{2 \pi i}{\lambda} \cdot\left(\frac{x x^{\prime}+y y^{\prime}}{R}\right)\right] \cdot d x \cdot d y .
\end{gathered}
$$

This factorization, of course, is a consequence of the fact that for complete coherence the angular bracket average, $\left\langle V_{\lambda}^{*}\left(P_{1}\right) \cdot V_{\lambda}\left(P_{2}\right)\right\rangle$, is simply equal to the product $V_{\lambda}^{*}\left(P_{1}\right) \cdot V_{\lambda}\left(P_{2}\right)$, so that the propagation of $V_{\lambda}\left(P_{1}\right)$ can be treated independently of $P_{2}$ and vice versa. In this example

$$
\begin{aligned}
& \mathrm{V}_{\lambda}\left(\mathrm{P}_{1}\right)=\mathrm{A}_{\mathrm{o}} \cdot \exp \left(\frac{2 \pi \mathrm{i}}{\lambda} \cdot \mathrm{x}_{1} \cdot \sin \theta_{0}\right) \\
& \qquad \text { for }-\mathrm{a}<\mathrm{x}_{1}<\mathrm{a} \text { and }-\mathrm{b}<\mathrm{y}_{1}<\mathrm{b} \\
& \text { and } \mathrm{v}_{\lambda}\left(\mathrm{P}_{1}\right)=0 \text { otherwise, }
\end{aligned}
$$

and eq. (10.62) simply describes the propagation of this field. In addition to this factorization the integrals factor into separate $x-$ and $y$-dependent integrals because we are considering rectangular apertures. Thus 


$$
v_{\lambda}\left(x^{\prime}, y^{\prime}, z^{\prime}\right)=\frac{z^{\prime}}{\lambda R^{2}} \cdot \exp \left(\frac{2 \pi i R}{\lambda}\right) \cdot I_{x} \cdot I_{y}
$$

where

$$
I_{x}=\int_{-a}^{a} \sqrt{A_{0}} \cdot \exp \left(\frac{2 \pi i}{\lambda} \cdot x \cdot \sin \theta_{0}\right) \cdot \exp \left(-\frac{2 \pi i}{\lambda} \cdot \frac{x x^{\prime}}{R}\right) \cdot d x
$$

and

$$
I_{y}=\int_{-b}^{b} \sqrt{A_{o}} \cdot \exp \left(-\frac{2 \pi i y y^{\prime}}{\lambda R}\right) \cdot d y
$$

These integrals are similar to those we evaluated for the incoherent source, so we can write them immediately as

$$
I_{x}=2 a \cdot \sqrt{A_{0}} \cdot \operatorname{sinc}\left[\frac{2 a}{\lambda} \cdot\left(\frac{x^{\prime}}{R}-\sin \theta_{0}\right)\right]
$$

and

$$
I_{y}=2 b \cdot \sqrt{A_{0}} \cdot \operatorname{sinc}\left(\frac{2 b y^{\prime}}{\lambda R}\right)
$$

Putting these expressions into eqs. (10.64) and (10.61), we finally obtain

$$
\begin{aligned}
& \mathrm{W}_{\lambda}\left(\mathrm{x}_{1}^{\prime}, \mathrm{y}_{1}^{\prime}, \mathrm{z}_{1}^{\prime} ; \mathrm{x}_{2}^{\prime}, \mathrm{y}_{2}^{\prime}, \mathrm{z}_{2}^{\prime}\right)=\frac{\mathrm{z}_{1}^{\prime} \mathrm{z}_{2}^{\prime} \mathrm{A}_{\mathrm{o}}{ }^{2}}{\lambda^{2} \mathrm{R}_{1}{ }^{2} \mathrm{R}_{2}{ }^{2}} \cdot 16 \mathrm{a}^{2} \mathrm{~b}^{2} \cdot \exp \left[\frac{2 \pi \mathrm{i}}{\lambda} \cdot\left(\mathrm{R}_{1}-\mathrm{R}_{2}\right)\right] \\
& \cdot \operatorname{sinc}\left[\frac{2 a}{\lambda} \cdot\left(\frac{x_{1}^{\prime}}{R_{1}}-\sin \theta_{0}\right)\right] \cdot \operatorname{sinc}\left[\frac{2 a}{\lambda} \cdot\left(\frac{x_{2}^{\prime}}{R_{2}}-\sin \theta_{0}\right)\right] \\
& \cdot \operatorname{sinc}\left(\frac{2 b y_{1}^{\prime}}{\lambda R_{1}}\right) \cdot \operatorname{sinc}\left(\frac{2 b y_{2}^{\prime}}{\lambda R_{2}}\right)
\end{aligned}
$$

for the cross-spectral density at the pair of points $P_{1}^{\prime}\left(x_{1}^{\prime}, y_{1}^{\prime}, z_{1}^{\prime}\right)$ and $P_{2}^{\prime}\left(x_{2}^{\prime}, y_{2}^{\prime}, z_{2}^{\prime}\right)$ produced by a uniform coherent beam passing through $a 2 a$ by $2 b$ rectangular aperture in the $X-Y$ plane with angle of incidence $\theta_{0}$. The spectral fluence rate at the point $P^{\prime}\left(x^{\prime}, y^{\prime}, z^{\prime}\right)$, obtained by setting $x_{2}^{\prime}=x_{1}^{\prime}=x^{\prime}$, etc., is

$F_{t, \lambda}\left(x^{\prime}, y^{\prime}, z^{\prime}\right)=\frac{z^{\prime 2}}{R^{4} \lambda^{2}} \cdot 16 a^{2} b^{2} \cdot A_{o}^{2} \cdot \operatorname{sinc}^{2}\left[\frac{2 a}{\lambda} \cdot\left(\frac{x^{\prime}}{R}-\sin \theta_{0}\right)\right] \cdot \operatorname{sinc}^{2}\left(\frac{2 b y^{\prime}}{\lambda R}\right) \cdot$

If we use this expression to evaluate $\sqrt{F_{t, \lambda}\left(P_{1}^{\prime}\right)}$ and $\sqrt{F_{t, \lambda}\left(P_{2}^{\prime}\right)}$ and then calculate the complex degree of coherence from eq. (10.26) using eq. (10.68) we find

$$
\mu\left(P_{1}^{\prime}, P_{2}^{\prime}\right)=\exp \left[\frac{2 \pi i}{\lambda} \cdot\left(R_{1}-R_{2}\right)\right]
$$

and

$$
\left|\mu\left(P_{1}^{\prime}, P_{2}^{\prime}\right)\right|=1
$$


The radiation is still completely coherent. The only way to reduce the degree of coherence is by somehow physically changing the phase relationships so that some averaging will take place in the angular bracket expression of eq. (10.14). Since this average is over both area and time, the degree of coherence can be reduced by randomizing the phases in either of these radiometric dimensions. If the instrumental spatial resolution is not too fine, coherent light scattered from a matte surface will be interpreted as incoherent since many speckles ${ }^{l}$ will be included in the instrumental resolution area. On the other hand if the instrumental spatial resolution is finer than the speckle size but its temporal resolution is not too fine then motion of the scattering matte surface will also introduce incoherence into the measurement by averaging over many speckles due to speckle motion during the measurement time interval. In either case, with better instrumental resolution the radiation would be found to be completely coherent -- although if the resolution, required to detect the coherence, results in an unmeasurably weak signal it serves no useful purpose to think of it as coherent.

The spectral fluence rate of eq. (10.69) describes the diffraction pattern associated with a rectangular aperture. The pattern is centered at $x^{\prime}=R \cdot \sin \theta_{0}, y^{\prime}=0$. It is useful to write the argument of the sinc function of eqs. (10.68) and (10.69) in terms of small angular displacements from the beam direction $\theta_{0}$. If we write

$$
\begin{aligned}
& \sin \theta=x^{\prime} / R \\
& \text { and } \alpha=\theta-\theta_{0} \\
& \text { we have } \quad \frac{2 a}{\lambda} \cdot\left(\frac{x^{\prime}}{R}-\sin \theta_{0}\right)=\frac{2 a}{\lambda} \cdot\left(\sin \theta-\sin \theta_{0}\right) .
\end{aligned}
$$

We can expand $\sin \theta$ in a Taylor series about $\theta_{0}$ to permit approximating $\sin \theta$ by

$$
\begin{aligned}
\sin \theta & =\sin \theta_{0}+\left(\theta-\theta_{0}\right) \cdot \cos \theta_{0}+\ldots . \\
& =\sin \theta_{0}+\alpha \cdot \cos \theta_{0} .
\end{aligned}
$$

Thus eq. (10.72) becomes

$$
\frac{2 \mathrm{a}}{\lambda} \cdot\left(\frac{\mathrm{x}^{\prime}}{\mathrm{R}}-\sin \theta_{0}\right)=\frac{2 \mathrm{a} \cdot \cos \theta_{0}}{\lambda} \cdot \alpha
$$

and the fluence rate, eq. $(10.69)$ can be written

$$
\mathrm{F}_{\mathrm{t}, \lambda}(\alpha, \beta)=\frac{\mathrm{z}^{\prime 2}}{\mathrm{R}^{4} \lambda^{2}} \cdot 16 \mathrm{a}^{2} \mathrm{~b}^{2} \cdot \mathrm{A}_{\mathrm{o}}{ }^{2} \cdot \operatorname{sinc}^{2}\left(\frac{2 \mathrm{a} \cdot \cos \theta_{0}}{\lambda} \cdot \alpha\right) \cdot \operatorname{sinc}{ }^{2}\left(\frac{2 \mathrm{~b}}{\lambda} \cdot \beta\right),
$$

where we have defined the angle $\beta$ by $\beta \simeq y^{\prime} / R$. In this form the equation shows that the angular distribution of radiation is the same as if an aperture of dimensions $2 a \cdot \cos \theta_{0} b y \quad 2 b$ were irradiated at normal incidence. Thus, at least for small angular deviations from the undiffracted beam, we may replace tilted apertures by their projections normal to the beam.

\footnotetext{
${ }^{1}$ Speckle is the random interference pattern produced by coherent radiation reflected from a surface which is rough over distances of wavelengths. Individual spots of the speckle pattern differ from one another in electric field direction (which we ignore in this scalar theory), amplitude, relative phase of the sinusoidal electric field vibration, and, of course, size and shape.
} 
MODELS for the CROSS-SPECTRAL DENSITY FUNCTION -- QUASIHOMOGENEITY. In principle it is possible to measure the cross-spectral density function. Any radiometric measurement provides some information about it. For example, the expression [eq. (10.20)] for the spectral radiance as a function of cross-spectral density can be inverted to give

$$
\begin{aligned}
w_{\lambda}(x+\xi / 2, y+n / 2 ; x-\xi / 2, y-n / 2) & =\int_{0}^{2 \pi} \int_{0}^{\pi / 2} L_{\lambda}(x, y, \theta, \phi) \\
& \cdot \exp [2 \pi i \cdot \sin \theta \cdot(\xi \cos \phi+n \sin \phi) / \lambda] \cdot \sin \theta \cdot d \theta \cdot d \phi .
\end{aligned}
$$

This shows how spectral radiance measurements can be employed to calculate the cross-spectral density. All that is needed is to measure the spectral radiance at a point ( $x, y$ ) from a great distance ${ }^{1}$ as a function of direction $(\theta, \phi)$ from that point and then evaluate the integrals for any desired pair of points symmetrically placed around ( $x, y)$. By varying $\xi$ and $\eta$ all such pairs of points can be covered. Finally, by remeasuring at other points $(x, y)$ the entire field can be mapped out. From a practical point of view this requires an almost prohibitive number of measurements and, moreover, because of the dependence of the integrand upon $\sin \theta$ the measurements at high angles become most important. As far as we are aware this technique has never been applied. More commonly the cross-spectral density is obtained from the measurement of more conventional interference effects. A number of references to such measurements are given in part II of reference [10.5].

Usually, if the cross-spectral density must be known, a reasonable functional form is assumed and then a small number of measurements of diffraction or interference effects will establish the values of any unknown parameters of the functional form. We have already seen a couple of functional forms which are commonly used for the cross-spectral density function: the delta function for incoherent radiation [eq. (10.28)] and models for completely coherent radiation [eqs. (10.32) and (10.59)]. All common models [10.3], [10.8], and [10.13] for the cross-spectral density function of partially coherent fields assume that the complex degree of coherence is a stationary function, depending only upon the distance between the points $P_{1}$ and $\mathrm{P}_{2}$. That is, it is assumed that $\mu\left(\mathrm{P}_{1}, \mathrm{P}_{2}\right)$ can be written ${ }^{2}$ as a function $g(\xi, \eta)$ where $\xi$ and $n$ are difference coordinates defined by

$$
\xi=x_{1}-x_{2} \text { and } n=y_{1}-y_{2}
$$

Thus we would write

$$
W_{\lambda}\left(P_{1}, P_{2}\right)=\sqrt{F_{t, \lambda}\left(P_{1}\right) \cdot F_{t, \lambda}\left(P_{2}\right)} \cdot g(\xi, n)
$$

This is known as the Schell model. A common form for $g$ is given by

$$
g(\xi, \eta)=\sin (\pi \rho / \sigma) /(\pi \rho / \sigma)
$$

where

$$
\rho^{2}=\xi^{2}+\eta^{2}
$$

\footnotetext{
${ }^{1}$ See footnote on page 48 .
}

${ }^{2}$ In general $\mu$ and $g$ will depend upon $\lambda$ as well as upon the coordinates of the two points. For simplicity we don't explicitly indicate this dependence in our notation. 
In this expression the parameter $\sigma$ is to be adjusted for best agreement with measured diffraction or interference effects. This form for $g$, with $\sigma$ taken as $\sim \lambda / 2$, provides a model for a real 'incoherent' source which will be lambertian and thus will describe the field over wide angles more realistically than the delta function model can. Another commonly used form for the degree of coherence is the Gaussian:

$$
g(\xi, \eta)=\exp \left[-\left(\xi^{2}+\eta^{2}\right) /\left(2 \sigma^{2}\right)\right]=\exp \left[-\rho^{2} /\left(2 \sigma^{2}\right)\right]
$$

As in the previous expression the adjustable parameter $\sigma$ is approximately equal to the coherence interval.

If in the Schell model, $\mu\left(\mathrm{P}_{1}, \mathrm{P}_{2}\right)$ decreases to zero with increasing separation of $\mathrm{P}_{1}$ and $P_{2}$ sufficiently rapidly that $F_{t, \lambda}\left(P_{1}\right) \sim F_{t, \lambda}\left(P_{2}\right)$ over the region where $\mu$ differs significantly from zero, or, putting it another way, if $F_{t, \lambda}(P)$ changes very slowly with position compared with $\mu$, then, in the expression (10.78) for cross-spectral density, we can approximate

$$
\sqrt{F_{t, \lambda}\left(P_{1}\right) \cdot F_{t, \lambda}\left(P_{2}\right)}=F_{t, \lambda}(x, y)
$$

where the average coordinates $x$ and $y$ are defined by

$$
x=\left(x_{1}+x_{2}\right) / 2 \text { and } y=\left(y_{1}+y_{2}\right) / 2
$$

Then the cross-spectral density can be expressed as the product of the spectral fluence rate by a complex degree of coherence:

$$
w_{\lambda}\left(P_{1}, P_{2}\right)=F_{t, \lambda}(x, y) \cdot g(\xi, \eta)
$$

This is known as the quasihomogeneous model. This model is important because most of the radiometric properties of a quasihomogeneous source are in accord with the ideas of classical (geometrical optics) radiometry. (For example, radiance is never negative and is, to a good approximation, invariant along a ray [10.14]). This is because, although a quasihomogeneous source may be highly coherent locally, the coherence areas are small compared to any brightness features on the surface so that the source, taken in its entirety, in many respects still behaves like a classical (incoherent) source.

Another useful feature of the quasihomogeneous model is that if a source is quasihomogeneous then the distant field from that source is also approximately quasihomogeneous. To show this let us consider eq. (10.42). We must, however, remark before doing this that the validity of the Fraunhofer approximation is even more strained here than in many coherence calculations because, in addition to requiring that $\sqrt{\lambda R}$ be large compared to the source dimension, we require that the source dimension be large compared to the coherence interval which, itself, can be taken as larger than $\lambda$ (otherwise we could simply treat the source as incoherent). We will assume that the points $P_{1}^{\prime}$ and $P_{2}^{\prime}$ lie near the z-axis on the surface of a large sphere centered at the origin so that we may take $R_{1}=R_{2}=R$ and $z_{i}^{\prime}=z_{2}^{\prime}=z^{\prime}$. Then we solve eqs. (10.77) and (10.82) for $x_{1}$ and $x_{2}$ in terms of $x$ and $\xi$, etc.

$$
\begin{array}{ll}
x_{1}=x+\xi / 2 & y_{1}=y+n / 2 \\
x_{2}=x-\xi / 2 & y_{2}=y-n / 2
\end{array}
$$


and substitute into eq. (10.42), making analogous substitutions for the primed variables ${ }^{2}$ :

$$
\begin{aligned}
W_{\lambda}\left(P_{i}^{\prime}, P_{2}^{\prime}\right)= & \frac{z^{\prime 2}}{R^{4} \lambda^{2}} \cdot \iiint \int_{\text {source }} w_{\lambda}\left(x+\frac{1}{2} \xi, y+\frac{1}{2} n ; x-\frac{1}{2} \xi, y-\frac{1}{2} n\right) \\
& \cdot \exp \left[-\frac{2 \pi i}{\lambda R} \cdot\left(\xi x^{\prime}+\xi^{\prime} x+n y^{\prime}+n^{\prime} y\right)\right] \cdot d x \cdot d y \cdot d \xi \cdot d n \\
= & \frac{z^{\prime 2}}{R^{4} \lambda^{2}} \cdot \iint_{\text {source }} F_{t, \lambda}(x, y) \cdot \exp \left[-\frac{2 \pi i}{\lambda R} \cdot\left(\xi^{\prime} x+n^{\prime} y\right)\right] \cdot d x \cdot d y \\
& \cdot \iint_{\text {source }} g(\xi, n) \cdot \exp \left[-\frac{2 \pi i}{\lambda R} \cdot\left(\xi x^{\prime}+n y^{\prime}\right)\right] \cdot d \xi \cdot d n
\end{aligned}
$$

where we have used eq. (10.83) for the explicit representation of the quasihomogeneous source. The limits of integration in the space of the average and difference coordinates are different than in the $x_{1}, y_{1}, x_{2}, y_{2}$ space. For example, for a rectangular source where the limits on $x_{1}$ and $x_{2}$ are $\pm a$ we could have [from eqs. (10.77) and (10.82)] $\xi$ run from $2|x|-2 a$ to $2 a-2|x|$ and $x$ run from $-a$ to $+a$. For a quasihomogeneous source the range over which $g$ differs significantly from zero is so small compared to the source dimensions that no appreciable error is introduced by replacing the $\xi$ and $\eta$ integration limits by the same limits that $x_{1}$ and $y_{1}$ are subject to or even possibly by $\pm \infty$ if convenient. In other words, in comparison to the size of the source, $g$ resembles roughly a delta function

${ }^{1}$ It is not obvious that the elements of integration are related by

$$
d x_{1} \cdot d y_{1} \cdot d x_{2} \cdot d y_{2}=d x \cdot d y \cdot d \xi \cdot d \eta
$$

This equality follows from the fact that the Jacobian of the coordinate transformation given by eqs. (10.77) and $(10.82)$ is 1 . The Jacobian of a transformation such as this is the determinant

$$
\frac{\partial\left(x_{1}, y_{1}, x_{2}, y_{2}\right)}{\partial\left(x_{1}, \xi, \eta\right)}=\left|\begin{array}{llll}
\frac{\partial x_{1}}{\partial x} & \frac{\partial x_{1}}{\partial y} & \frac{\partial x_{1}}{\partial \xi} & \frac{\partial x_{1}}{\partial \eta} \\
\frac{\partial y_{1}}{\partial x} & \frac{\partial y_{1}}{\partial y} & \frac{\partial y_{1}}{\partial \xi} & \frac{\partial y_{1}}{\partial \eta} \\
\frac{\partial x_{2}}{\partial x} & \frac{\partial x_{2}}{\partial y} & \frac{\partial x_{2}}{\partial \xi} & \frac{\partial x_{2}}{\partial \eta} \\
\frac{\partial y_{2}}{\partial x_{2}} & \frac{\partial y_{2}}{\partial y} & \frac{\partial y_{2}}{\partial \xi} & \frac{\partial y_{2}}{\partial \eta}
\end{array}\right|
$$

This expresses how the multidimensional element of integration transforms under the coordinate transformation, i.e.,

$$
d x_{1} \cdot d y_{1} \cdot d x_{2} \cdot d y_{2}=\frac{\partial\left(x_{1}, y_{1}, x_{2}, y_{2}\right)}{\partial\left(x, y, y_{1}, n\right)} \cdot d x \cdot d y \cdot d \xi \cdot d n
$$

[For more details see any text on advanced calculus.] 
so that, beyond a certain point, the limits of integration of any integral involving it are largely irrelevant. In our rectangular source example, then, we could take the limits of the $\xi$ integration also to be $\pm \mathrm{a}$, for instance, without significant error. Allowing further approximations of this sort then, we see that the left hand side of eq. (10.88) is a product of two independent functions defined by double integrals: the first, a function only of the difference coordinates $\xi^{\prime}$ and $\eta^{\prime}$ in the observation surface, and the second, a function only of the average coordinates $x^{\prime}$ and $y^{\prime}$ in the observation surface. So we evidently can write

$$
w_{\lambda}\left(x_{1}^{\prime}, y_{1}^{\prime}, z^{\prime} ; x_{2}^{\prime}, y_{2}^{\prime}, z^{\prime}\right)=F_{t, \lambda}^{\prime}\left(x^{\prime}, y^{\prime}\right) \cdot g^{\prime}\left(\xi^{\prime}, n^{\prime}\right),
$$

where

$$
\begin{aligned}
& g^{\prime}\left(\xi^{\prime}, \eta^{\prime}\right)=\frac{1}{N_{\lambda}} \cdot \iint_{\text {source }} F_{t, \lambda}(x, y) \cdot \exp \left[-\frac{2 \pi i}{\lambda R} \cdot\left(\xi^{\prime} x+n^{\prime} y\right)\right] \cdot d x \cdot d y, \\
& F_{t, \lambda}^{\prime}\left(x^{\prime}, y^{\prime}\right)=N_{\lambda} \cdot\left(\frac{z^{\prime}}{\lambda R^{2}}\right)^{2} \cdot \iint_{\text {source }} g(\xi, n) \cdot \exp \left[-\frac{2 \pi i}{\lambda R} \cdot\left(\xi x^{\prime}+n y^{\prime}\right)\right] \cdot d \xi \cdot d \eta,
\end{aligned}
$$

and

$$
\mathrm{N}_{\lambda}=\iint_{\text {source }} F_{t, \lambda}(x, y) \cdot d x \cdot d y .
$$

This shows that the cross-spectral density in the observation surface is also approximately quasihomogeneous at least within the validity of the Fraunhofer approximation. In deriving this result we assumed that $R_{1}=R_{2}=R$ and $z_{i}^{\prime}=z_{2}^{\prime}=z^{\prime}$. We assume that these will still be good approximations and our conclusions valid for field points near the $z$-axis in any plane parallel to the $x-y$ plane provided the distance, $z$ ', from the source is large compared to the coherence intervals there. The field from a quasihomogeneous source will not be quasihomogeneous beyond an aperture which is not large compared to the coherence area at that aperture. At such an aperture the condition that $F_{t, \lambda}(P)$ be slowly changing compared to $\mu$ is not fulfilled and this aperture then constitutes a non-quasihomogeneous source for the field points beyond it.

Equations (10.90) represent a simplified propagation law for quasihomogeneous fields. We notice that the complex degree of coherence in the source determines the spectral fluence rate and hence the directionality of the distant field. Conversely, the distribution of spectral fluence rate or radiance in the source determines the degree of coherence in the field. Frequently only the second of eqs. (10.90) will be required and this results in a further simplification of the propagation law. For example, a rectangular, uniform, incoherent source [eq. (10.43)] is quasihomogeneous. If, within the rectangle of the source, we identify

$$
F_{t, \lambda}=A_{0}^{2} \text { and } g=\lambda^{2} \cdot \delta(\xi) \cdot \delta(\eta)
$$

Then, from the second of eqs. (10.90), we immediately obtain eq. (10.50) for the spectral fluence rate at a distance from such a source: 


$$
\begin{aligned}
F_{t, \lambda}^{\prime}\left(x^{\prime}, y^{\prime}\right) & =N_{\lambda} \cdot\left(\frac{z^{\prime}}{\lambda R^{2}}\right)^{2} \cdot \lambda^{2} \\
& =4 a b \cdot A_{0}^{2} \cdot \cos ^{2} \theta / R^{2} .
\end{aligned}
$$

We will not make much use of the quasihomogeneous approximation in this chapter because we will normally wish to present calculations covering the entire range from incoherent to coherent to the same degree of approximation. However, it should be apparent that this model is extremely useful in many problems involving fields originating in thermal sources.

The EFFECTS of IMAGING or FOCUSING OPTICS. The preceding examples of the propagation of the cross-spectral density function illustrate the meanings of the variables and the kinds of approximations which are frequently made in evaluating the integrals. Usually, of course, one is not interested in simple, unobstructed propagation from a source to a detector but rather propagation through a series of lenses and apertures and what-not to a final detector. To handle such a situation one must sequentially apply the propagation equation [eq. (10.36)] from the plane of each aperture which might restrict the beam to the plane of the next one so that the cross-spectral density calculated for one surface becomes the source for the next calculation, and so on until finally the detector is reached. Clearly, for a complicated optical system, evaluating the multiple integrals involved will become a chore. Fortunately, one of the most important optical systems -- a simple source-lens-image system -- is not significantly more difficult than the surface irradiation examples we have just treated so we will turn now to that problem.

We will consider the propagation of the cross-spectral density from the X-Y source plane to a spherical surface of radius $R$ centered at the origin (figure 10.6a). We can think of this surface, for example, as a spherical mirror. Then we will consider the further propagation of the cross-spectral density from this surface to the image plane. The first part of this task is accomplished by setting $R_{1}=R_{2}=R$ in eq. (10.42):

$$
\begin{aligned}
w_{\lambda}^{\prime}\left(x_{1}^{\prime}, y_{1}^{\prime} ; x_{2}^{\prime}, y_{2}^{\prime}\right)= & \frac{\cos \theta_{1} \cdot \cos \theta_{2}}{R^{2} \lambda^{2}} \cdot \iiint \int_{\text {source }} w_{\lambda}\left(x_{1}, y_{1} ; x_{2}, y_{2}\right) \\
& \cdot \exp \left[-\frac{2 \pi i}{\lambda R} \cdot\left(x_{1} x_{1}^{\prime}+y_{1} y_{1}^{\prime}-x_{2} x_{2}^{\prime}-y_{2} y_{2}^{\prime}\right)\right] \cdot d x_{1} \cdot d y_{1} \cdot d x_{2} \cdot d y_{2} \cdot
\end{aligned}
$$

In the integrations, points $\mathrm{P}_{1}\left(\mathrm{x}_{1}, \mathrm{y}_{1}\right)$ and $\mathrm{P}_{2}\left(\mathrm{x}_{2}, \mathrm{y}_{2}\right)$ each independently cover the entire source area in the $X-Y$ plane. The points $P_{1}^{\prime}$ and $P_{2}^{\prime}$, for which the new cross-spectral density on the left is calculated, lie in the spherical surface of radius R. Actually the surface need not be spherical. All we require is that the optical path length from the origin along all geometrical optic rays to the surface be constant. Therefore this surface could be the plane through the center of a lens (figure 10.6c). On the image side of this "spherical" surface we shall assume the radius is R", thus allowing for the possibility of imaging with a magnification other than one. For the propagation from this surface to the image plane, then, we start with eq. (10.36) and approximate the distance s" (figure 10.6b) from the surface to points $P^{\prime \prime}$ in the image plane by [see eq. (10.39)]

$$
s^{\prime \prime} \approx R^{\prime \prime}-\left[\left(x^{\prime \prime} x^{\prime}+y^{\prime \prime} y^{\prime}\right) / R^{\prime \prime}\right]+\frac{1}{2}\left(x^{\prime \prime}+y^{\prime \prime}\right) / R^{\prime \prime}
$$



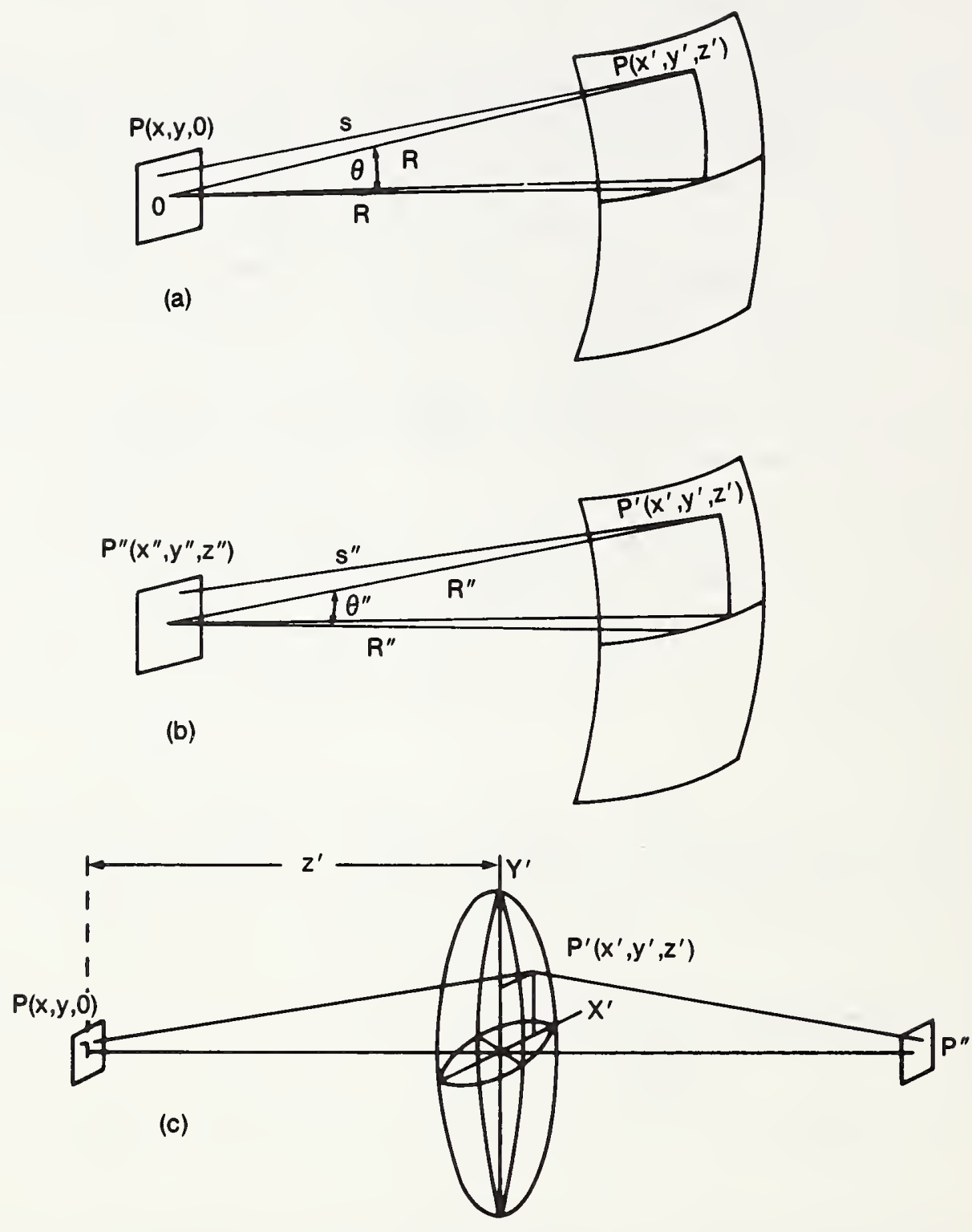

Figure 10.6. (a) Cross-spectral density propagation from source plane to intermediate optical element (mirror).

(b) Cross-spectral density propagation from intermediate optical element (mirror) to detector plane.

(c) Cross-spectral density propagation from source plane through intermediate optical element (lens) to detector plane. 
and finally obtain

$$
\begin{aligned}
W_{\lambda}^{\prime \prime}\left(x_{1}^{\prime \prime}, y_{1}^{\prime \prime} ; x_{2}^{\prime \prime}, y_{2}^{\prime \prime}\right) & =\frac{\cos \theta_{1}^{\prime} \cdot \cos \theta_{2}^{\prime}}{\mathrm{R}^{\prime \prime} \cdot \lambda^{2}} \cdot \exp \left[\frac{\pi i}{\lambda R^{\prime \prime}} \cdot\left(x_{1}^{\prime \prime}+y_{1}^{\prime \prime 2}-x_{2}^{\prime \prime 2}-y_{2}^{\prime \prime 2}\right)\right] \cdot \iint_{\text {aperture }} w_{\lambda}^{\prime}\left(x_{1}^{\prime} \cdot y_{1}^{\prime} ; x_{2}^{\prime} \cdot y_{2}^{\prime}\right) \\
& \cdot \exp \left[-\frac{2 \pi i}{\lambda R^{\prime \prime}} \cdot\left(x_{1}^{\prime} x_{1}^{\prime \prime}+y_{1}^{\prime} y_{1}^{\prime \prime}-x_{2}^{\prime} x_{2}^{\prime \prime}-y_{2}^{\prime} y_{2}^{\prime \prime}\right)\right] \cdot d x_{1}^{\prime} \cdot d y_{1}^{\prime} \cdot d x_{2}^{\prime} \cdot d y_{2}^{\prime},
\end{aligned}
$$

where we have, as usual, approximated $s_{1}^{\prime \prime} \cdot s_{2}^{\prime \prime}$ in the denominator by $\mathrm{R}^{\prime 2}$. In the integrations of eq. (10.95) each point $P_{i}^{\prime}\left(x_{1}^{\prime}, y_{1}^{\prime}\right)$ and $P_{2}^{\prime}\left(x_{2}^{\prime}, y_{2}^{\prime}\right)$ covers the entire spherical surface independently. We will assume that there is a rectangular aperture at this focusing optic which limits this integration to a $2 a^{\prime}$ by $2 b^{\prime}$ area. The points $P_{1}^{\prime \prime}$ and $P_{2}^{\prime \prime}$ of the cross-spectral density function on the left are points in the image plane.

The next task is to substitute eq. (10.93) into eq. (10.95). First we will drop the exponential factor outside the integral in eq. (10.95). The points of primary interest in the image plane lie near the image of the source and since source points in the Fraunhofer approximation satisfy $\left(x^{2}+y^{2}\right) /(\lambda R) \ll 1$ the points of interest in the image plane will satisfy:

$$
\frac{x^{\prime 2}+y^{\prime 2}}{\lambda R^{\prime \prime}}=\frac{\left(x R^{\prime \prime} / R\right)^{2}+\left(y R^{\prime \prime} / R\right)^{2}}{\lambda R^{\prime \prime}}=\frac{x^{2}+y^{2}}{\lambda R}\left(R^{\prime \prime} / R\right) \ll R^{\prime \prime} / R
$$

where $R^{\prime \prime} / R$ is the optical system magnification. Thus, the quadratic phase factor

$$
\exp \left[\frac{\pi i}{\lambda R^{\prime \prime}}\left(x_{1}^{\prime \prime 2}+y_{1}^{\prime 2}-x_{2}^{\prime 2}-y_{2}^{\prime 2}\right)\right]
$$

will be unity except for very large system magnifications or image plane points very distant from the $z$-axis. Second, the $\operatorname{cosine}$ factors, $\cos \theta_{1}, \cos \theta_{2}, \cos \theta_{1}$, and $\cos \theta_{2}$ will prove to be a nuisance in what is to follow so we will now make one final approximation and set all these factors equal to 1 . The angles ${ }_{1}$ and ${ }_{2}$ are the angles at the object plane between the $\mathrm{Z}$-axis and the directions to the points $\mathrm{P}_{1}^{\prime}$ and $\mathrm{P}_{2}^{\prime}$ in the spherical surface, and similarly $\theta_{i}$ and $\theta_{2}$ are the corresponding angles at the spherical surface between the normals to the spherical surface at $P_{1}^{\prime}$ and $P_{2}^{\prime}$ and the directions to $P_{1}^{\prime \prime}$ and $P_{2}^{\prime \prime}$ respectively. So replacing these factors by 1 means that we are assuming a small aperture at the lens or mirror, i.e., $a^{\prime}$ and $b^{\prime}$ are small compared to $R$ and $R^{\prime \prime}$. With these approximations, and reversing the order to integrate first over the aperture and then over the source, we obtain:

$$
\begin{aligned}
w_{\lambda}^{\prime \prime}\left(x_{1}^{\prime \prime}, y_{1}^{\prime \prime} ; x_{2}^{\prime \prime}, y_{2}^{\prime \prime}\right)= & \frac{1}{R^{2} \cdot R^{\prime \prime} \cdot \lambda^{4}} \cdot \iiint \int_{\text {source }} w_{\lambda}\left(x_{1}, y_{1} ; x_{2}, y_{2}\right) \\
& \cdot\left\{\iiint_{\substack{\text { apens } \\
\text { aperture }}} \exp \left[-\frac{2 \pi i}{\lambda R} \cdot\left(x_{1} x_{1}^{\prime}+y_{1} y_{1}^{\prime}-x_{2} x_{2}^{\prime}-y_{2} y_{2}^{\prime}\right)\right]\right. \\
& \left.\cdot \exp \left[-\frac{2 \pi i}{\lambda R^{\prime \prime}} \cdot\left(x_{1}^{\prime} x_{1}^{\prime \prime}+y_{1}^{\prime \prime} y_{1}^{\prime}-x_{2}^{\prime} x_{2}^{\prime \prime}-y_{2}^{\prime} y_{2}^{\prime \prime}\right)\right] \cdot d x_{1}^{\prime} \cdot d y_{1}^{\prime} \cdot d x_{2}^{\prime} \cdot d y_{2}^{\prime}\right\} \cdot d x_{1} \cdot d y_{2} \cdot d x_{1} \cdot d y_{2} \cdot
\end{aligned}
$$


Thanks to our having chosen a rectangular aperture and having eliminated all the other factors which depend upon $x_{i}^{\prime}, y_{1}^{\prime}, x_{2}^{\prime}$, and $y_{2}^{\prime}$ the integrations over these singly primed variables can be carried out and (Surprise!) lead again to sinc functions:

$$
\begin{aligned}
\mathrm{W}_{\lambda}^{\prime \prime}\left(\mathrm{x}_{1}^{\prime \prime}, \mathrm{y}_{1}^{\prime \prime} ; \mathrm{x}_{2}^{\prime \prime}, \mathrm{Y}_{2}^{\prime \prime}\right)= & \frac{1}{\mathrm{R}^{2} \cdot \mathrm{R}^{\prime 2} \cdot \lambda^{4}} \cdot \iiint \int_{\text {source }} \mathrm{W}_{\lambda}\left(\mathrm{x}_{1}, \mathrm{y}_{1} ; \mathrm{x}_{2}, \mathrm{y}_{2}\right) \\
& \cdot 16 \mathrm{a}^{\prime 2} \cdot \mathrm{b}^{\prime 2} \cdot \operatorname{sinc}\left[\frac{2 \mathrm{a}^{\prime}}{\lambda} \cdot\left(\frac{\mathrm{x}_{1}}{\mathrm{R}}+\frac{\mathrm{x}_{1}^{\prime \prime}}{\mathrm{R}^{\prime \prime}}\right)\right] \cdot \operatorname{sinc}\left[\frac{2 \mathrm{~b}^{\prime}}{\lambda} \cdot\left(\frac{\mathrm{Y}_{1}}{\mathrm{R}}+\frac{\mathrm{y}_{1}^{\prime \prime}}{\mathrm{R}^{\prime \prime}}\right)\right] \\
& \cdot \operatorname{sinc}\left[\frac{2 \mathrm{a}^{\prime}}{\lambda} \cdot\left(\frac{\mathrm{x}_{2}}{\mathrm{R}}+\frac{\mathrm{x}_{2}^{\prime \prime}}{\mathrm{R}^{\prime \prime}}\right)\right] \cdot \operatorname{sinc}\left[\frac{2 \mathrm{~b}^{\prime}}{\lambda} \cdot\left(\frac{\mathrm{Y}_{2}}{\mathrm{R}}+\frac{\mathrm{y}_{2}^{\prime \prime}}{\mathrm{R}^{\prime \prime}}\right)\right] \cdot d \mathrm{dx}_{1} \cdot d \mathrm{y}_{1} \cdot d \mathrm{dx}_{2} \cdot d \mathrm{y}_{2} \cdot
\end{aligned}
$$

Thus, the cross-spectral density in the image plane is given by an integral over the object plane of the cross-spectral density of the object weighted by a product of sinc functions.

Equations (10.98) and (10.99) have been derived in the Fraunhofer approximation which is perhaps rarely applicable in practical imaging situations. Fortunately, some of the higher order terms which we have neglected actually cancel out in the case of image formation so the approximation is not as bad as it might be. We will return later to a more general consideration of imaging and discuss a more exact equivalent of these equations which, in principle, can be applied to other aperture shapes and includes lens aberrations and focusing errors. The present approximation is generally suitable for very small sources such as pinholes and narrow slits but overstates diffraction effects for larger sources. For example, if the radiances at the centers of two circular sources of different sizes are to be measured by imaging them in turn onto a small detector located at the center of the image then the estimate of the contribution to the detector signal of the larger source caused by diffraction from the extra source size will be too large in this approximation. Usually, in relative measurements, such errors will be small and calculations in the Fraunhofer approximation will be adequate to establish the negligibility of the error or to indicate the measurement modifications required to make it negligible.

If the same derivation is carried out for a quasihomogeneous source using the propagation law of eqs. (10.90) and assuming that the $2 a^{\prime}$ by $2 b^{\prime}$ intermediate aperture at the focusing optic is large compared to the coherence area, then we find that the image is quasihomogeneous with cross-spectral density given by

$$
\begin{aligned}
W_{\lambda}^{\prime \prime}\left(x_{1}^{\prime \prime}, y_{1}^{\prime \prime} ; x_{2}^{\prime \prime}, y_{2}^{\prime \prime}\right) & =W_{\lambda}^{\prime \prime}\left(x^{\prime \prime}+\xi " / 2, y^{\prime \prime}+\eta^{\prime \prime} / 2 ; x^{\prime \prime}-\xi " / 2, y^{\prime \prime}-\eta " / 2\right) \\
& =F_{t, \lambda}^{\prime \prime}\left(x^{\prime \prime}, y^{\prime \prime}\right) \cdot g^{\prime \prime}\left(\xi ", \eta^{\prime \prime}\right),
\end{aligned}
$$

where

$$
\begin{aligned}
g^{\prime \prime}\left(\xi^{\prime \prime}, n^{\prime \prime}\right)= & \frac{1}{G} \cdot \iint_{\text {source }} g(\xi, n) \cdot 2 a^{\prime} \cdot \operatorname{sinc}\left[\frac{2 a^{\prime}}{\lambda} \cdot\left(\frac{\xi}{R}+\frac{\xi^{\prime \prime}}{R^{\prime \prime}}\right)\right] \cdot 2 b^{\prime} \cdot \operatorname{sinc}\left[\frac{2 b^{\prime}}{\lambda} \cdot\left(\frac{n}{R}+\frac{n^{\prime \prime}}{R^{\prime \prime}}\right)\right] \cdot d \xi \cdot d n, \\
F_{t, \lambda}^{\prime \prime}\left(x^{\prime \prime}, y^{\prime \prime}\right)= & G \cdot\left(\frac{z}{\lambda R^{2}}\right)^{2} \cdot\left(\frac{z^{\prime \prime}}{\lambda R^{\prime 2}}\right)^{2} \cdot \iint_{\text {source }} F_{t, \lambda}(x, y) \cdot 2 a^{\prime} \\
& \cdot \operatorname{sinc}\left[\frac{2 a^{\prime}}{\lambda} \cdot\left(\frac{x}{R}+\frac{x^{\prime \prime}}{R^{\prime \prime}}\right)\right] \cdot 2 b^{\prime} \cdot\left[\frac{2 b^{\prime}}{\lambda} \cdot\left(\frac{Y}{R}+\frac{y^{\prime \prime}}{R^{\prime \prime}}\right)\right] \cdot d x \cdot d y
\end{aligned}
$$

and 


$$
G=\iint_{\text {source }} g(\xi, n) \cdot 2 a^{\prime} \cdot \operatorname{sinc}\left(\frac{2 a^{\prime} \xi}{\lambda R}\right) \cdot 2 b^{\prime} \cdot \operatorname{sinc}\left(\frac{2 b^{\prime} n}{\lambda R}\right) \cdot d \xi \cdot d n \text {. }
$$

In these equations $x^{\prime \prime}, y^{\prime \prime}, \xi^{\prime \prime}$, and $\eta^{\prime \prime}$ are the average and difference coordinates in the image plane analogous to those defined in eqs. (10.77) and (10.82) for the source plane. The condition that the intermediate lens aperture be large compared to the coherence area at the aperture is almost invariably fulfilled in optical radiometry if the imagery is good and the source is quasihomogeneous. We can calculate the coherence area from the degree of coherence in the aperture which, in turn, is obtained by eq. (10.90) from the fluence rate distribution in the source. Assuming a roughly uniform source of dimensions $2 \mathrm{a}$ by $2 \mathrm{~b}$ we find that $g^{\prime}\left(\xi^{\prime}, n^{\prime}\right)$ is proportional to

$$
\operatorname{sinc}\left(2 a \xi^{\prime} / \lambda R\right) \cdot \operatorname{sinc}\left(2 b \eta^{\prime} / \lambda R\right)
$$

and hence the coherence area is roughly $0.4 \lambda^{2} R^{2} / a b$. [See eqs. (10.56) and (10.57)]. The condition on the aperture then becomes $4 \mathrm{a}^{\prime} \mathrm{b}^{\prime}>>0.4 \lambda^{2} \mathrm{R}^{2} / \mathrm{ab}$ or $\omega>>1.6 \lambda^{2} /(4 \mathrm{ab})$ where $\omega$ is the solid angle subtended at the source by the aperture, typically 0.01 [sr] or larger, and $4 \mathrm{ab}$ is the source area.

We will now illustrate the use of eq. (10.99) by applying it to the problem of imaging the same two sources we treated before, namely, a uniform rectangular incoherent source and a uniform rectangular coherent source. We have already seen [eqs. (10.47) and (10.68)] what the crossspectral density produced by these sources looks like in the plane of the imaging optics. Now we will see what happens when this radiation is brought to a focus. Since the equations are rapidly becoming inconveniently long we will treat only the $x$-component of the problem, recalling that in the Fraunhofer approximation with rectangular sources and apertures, the $x$ and $y$ parts of the integrals are entirely separate factors which can be evaluated independently of one another. Thus we will write just the $\mathrm{x}$-component of eq. (10.99) as

$$
\begin{gathered}
W_{\lambda}^{\prime \prime}(x)\left(x_{1}^{\prime \prime} ; x_{2}^{\prime \prime}\right)=\frac{4 a^{\prime 2}}{R R^{\prime \prime} \lambda^{2}} \cdot \iint_{\text {source }} W_{\lambda}^{(x)}\left(x_{1} ; x_{2}\right) \cdot \operatorname{sinc}\left[\frac{2 a^{\prime}}{\lambda} \cdot\left(\frac{x_{1}}{R}+\frac{x_{1}^{\prime \prime}}{R^{\prime \prime}}\right)\right] \\
\cdot \operatorname{sinc}\left[\frac{2 a^{\prime}}{\lambda} \cdot\left(\frac{x_{2}}{R}+\frac{x_{2}^{\prime \prime}}{R^{\prime \prime}}\right)\right] \cdot d x_{1} \cdot d_{2} \cdot
\end{gathered}
$$

There is an analogous $y$-dependent factor containing $b$ and $y$ instead of $a$ and $x$ and the complete cross-spectral density is understood to be a product of the two factors:

$$
\mathrm{w}_{\lambda}\left(\mathrm{x}_{1}, \mathrm{y}_{1} ; \mathrm{x}_{2}, \mathrm{y}_{2}\right)=\mathrm{w}_{\lambda}^{(\mathrm{x})}\left(\mathrm{x}_{1} ; \mathrm{x}_{2}\right) \cdot \mathrm{w}_{\lambda}^{(\mathrm{y})}\left(\mathrm{y}_{1} ; \mathrm{y}_{2}\right)
$$

In other coordinate systems, such as polar coordinates the integrals do not factor this way and it is necessary to start from the complete equation (10.98).

For the incoherent uniform rectangular source, we have from eq. (10.28)

$$
\begin{aligned}
\mathrm{W}_{\lambda}^{(\mathrm{x})}\left(\mathrm{x}_{1} ; \mathrm{x}_{2}\right) & =\lambda \mathrm{A}_{0} \cdot \delta\left(\mathrm{x}_{1}-\mathrm{x}_{2}\right) & & \text { for }-\mathrm{a}<\mathrm{x}_{1}<\mathrm{a} \\
& =0 & & \text { for }\left|\mathrm{x}_{1}\right| \geq \mathrm{a}
\end{aligned}
$$

and after substitution of this expression into eq. (10.105) and carrying out the integration over $x_{2}$ we are left with 


$$
\begin{aligned}
W_{\lambda}^{\prime \prime}(x)\left(x_{1}^{\prime \prime} ; x_{2}^{\prime \prime}\right)=\frac{4 a^{\prime 2} \cdot A_{0}}{R R^{\prime \prime} \lambda} & \cdot \int_{-a}^{a} \operatorname{sinc}\left[\frac{2 a^{\prime}}{\lambda} \cdot\left(\frac{x_{1}}{R}+\frac{x_{1}^{\prime \prime}}{R^{\prime \prime}}\right)\right] \\
\cdot \operatorname{sinc} & {\left[\frac{2 a^{\prime}}{\lambda} \cdot\left(\frac{x_{1}}{R}+\frac{x_{2}^{\prime \prime}}{R^{\prime \prime}}\right)\right] \cdot d x_{1} . }
\end{aligned}
$$

This integral, if needed, can be evaluated ${ }^{l}$ but frequently the fluence rate is of more interest:

$$
\begin{aligned}
& \mathrm{F}_{\mathrm{t}, \lambda}^{\prime \prime}(\mathrm{x})\left(\mathrm{x}^{\prime \prime}\right)=\mathrm{W}_{\lambda}^{\prime \prime}(\mathrm{x})\left(\mathrm{x}^{\prime \prime} ; \mathrm{x}^{\prime \prime}\right) \\
& =\frac{4 a^{\prime 2} \cdot A_{0}}{R R^{\prime \prime} \lambda} \cdot \int_{-a}^{a} \operatorname{sinc}^{2}\left[\frac{2 a^{\prime}}{\lambda} \cdot\left(\frac{x_{1}}{R}+\frac{x^{\prime \prime}}{R^{\prime \prime}}\right)\right] \cdot d x_{1} .
\end{aligned}
$$

The integral appearing in this equation can be evaluated in terms of tabulated functions with the result

${ }^{1}$ When the integration is carried out, the result is

$$
W_{\lambda}^{\prime \prime}(x)\left(x_{1}^{\prime \prime} ; x_{2}^{\prime \prime}\right)=\frac{A_{0} \lambda}{2 \pi^{2}}(L-C+S)
$$

where

$$
\begin{aligned}
& \mathrm{L}=\frac{\cos \left[\frac{2 \pi a^{\prime}}{\lambda R^{\prime \prime}} \cdot\left(x_{1}^{\prime \prime}-x_{2}^{\prime \prime}\right)\right]}{x_{1}^{\prime \prime}-x_{2}^{\prime \prime}} \cdot \ln \left(\frac{x_{2}^{\prime \prime} R+a R^{\prime \prime}}{x_{2}^{\prime \prime} R-a R^{\prime \prime}} \cdot \frac{x_{1}^{\prime \prime} R-a R^{\prime \prime}}{x_{1}^{\prime \prime} R+a R^{\prime \prime}}\right) \\
& C=\frac{\cos \left[\frac{2 \pi a^{\prime}}{\lambda R^{\prime \prime}} \cdot\left(x_{1}^{\prime \prime}-x_{2}^{\prime \prime}\right)\right]}{x_{1}^{\prime \prime}-x_{2}^{\prime \prime}} \cdot\left\{\operatorname{Ci}\left[U_{2}(+)\right]-\operatorname{Ci}\left[U_{2}(-)\right]-\operatorname{Ci}\left[U_{1}(+)\right]+\operatorname{Ci}\left[U_{1}(-)\right]\right\} \\
& S=\frac{\sin \left[\frac{2 \pi a^{\prime}}{\lambda R^{\prime \prime}} \cdot\left(x_{1}^{\prime \prime}-x_{2}^{\prime \prime}\right)\right]}{x_{1}^{\prime \prime}-x_{2}^{\prime \prime}} \cdot\left\{\operatorname{Si}_{2}\left[U_{2}(+)\right]-\operatorname{Si}\left[U_{2}(-)\right]+\operatorname{Si}\left[U_{1}(+)\right]-\operatorname{Si}\left[U_{1}(-)\right]\right\}
\end{aligned}
$$

and

$$
\begin{aligned}
& U_{k}(+)=\frac{4 \pi a^{\prime}}{\lambda} \cdot\left(\frac{x_{k}^{\prime \prime}}{R^{\prime \prime}}+\frac{a}{R}\right) \\
& U_{k}(-)=\frac{4 \pi a^{\prime}}{\lambda} \cdot\left(\frac{x_{k}^{\prime \prime}}{R^{\prime \prime}}-\frac{a}{R}\right) .
\end{aligned}
$$

Most of the integrals which arise in diffraction problems with simple rectangular and circular sources and apertures can be evaluated with the help of any of the references [10.15], [10.16] or [10.17]. Reference [10.15] also includes tables of numerical values of many of the functions which arise in these problems, such as $\mathrm{Ci}(\mathrm{x})$ and $\mathrm{Si}(\mathrm{x})$. These tabulations are useful for isolated calculations. Of probably more value, however, are the rational approximations presented in this reference which permit efficient computer evaluation of these functions with good accuracy. The brute force computer evaluation of multiple integrals is often sufficiently slow and uncertain as to warrant the expenditure of considerable effort to find physical idealizations and mathematical approximations which permit reduction of the integrals to a form which can be evaluated in terms of well-known functions with rational approximations. 


$$
\begin{aligned}
& F_{t, \lambda}^{\prime \prime}(x)=\frac{4 a^{\prime 2} \cdot A_{o}}{R R^{\prime \prime} \lambda} \cdot \frac{\lambda R}{2 \pi a^{\prime}} \cdot\left\{S i\left[\frac{4 \pi a^{\prime}}{\lambda} \cdot\left(\frac{x^{\prime \prime}}{R^{\prime \prime}}+\frac{a}{R}\right)\right]-S i\left[\frac{4 \pi a^{\prime}}{\lambda} \cdot\left(\frac{x^{\prime \prime}}{R^{\prime \prime}}-\frac{a}{R}\right)\right]\right. \\
& \left.+\frac{\sin ^{2}\left[\frac{2 \pi a^{\prime}}{\lambda} \cdot\left(\frac{x^{\prime \prime}}{R^{\prime \prime}}-\frac{a}{R}\right)\right]}{\frac{2 \pi a^{\prime}}{\lambda} \cdot\left(\frac{x^{\prime \prime}}{R^{\prime \prime}}-\frac{a}{R}\right)}-\frac{\sin ^{2}\left[\frac{2 \pi a^{\prime}}{\lambda} \cdot\left(\frac{x^{\prime \prime}}{R^{\prime \prime}}+\frac{a}{R}\right)\right]}{\frac{2 \pi a^{\prime}}{\lambda} \cdot\left(\frac{x^{\prime \prime}}{R^{\prime \prime}}+\frac{a}{R}\right)}\right\} \text {. }
\end{aligned}
$$

The function $S i(x)=\int_{0}^{x} \frac{\sin t}{t} \cdot d t$ is known as the sine integral and is tabulated in [10.15], where rational fraction approximations suitable for computer calculations are also given. The complete fluence rate, of course, is the product of the x-component of eq. (10.116) and a similar $y$-component in which $x$ and $a$ have been replaced by $y$ and $b$. The quantities $a$ and $b$ are the object half-dimensions and $a^{\prime}$ and $b^{\prime}$ are the aperture half-dimensions at the imaging optic. The quantity $R$ is the object-to-lens distance and $R^{\prime \prime}$ is the lens-to-image distance. The coordinates of the point of interest in the image plane are $x^{\prime \prime}$ and $y^{\prime \prime}$. In figure 10.7 we show some graphs of eq. (10.116) for several values of the lens aperture halfwidth $a^{\prime}$. This figure illustrates how the image, which ideally would be constant from $x^{\prime \prime}=-a R^{\prime \prime} / R$ to $x^{\prime \prime}=a R^{\prime \prime} / R$ and zero elsewhere, broadens by diffraction as the aperture is decreased. Of course we have assumed no imaging errors other than diffraction in eqs. (10.98) to (10.116).

To the extent that spectral fluence rate approximates spectral irradiance this is the distribution of spectral irradiance we would expect to find, for example, in the image of a tungsten strip filament lamp.

At the other extreme -- a coherent uniform rectangular source -- we have for the source cross-spectral density [eq. (10.59)]

$$
\begin{aligned}
W_{\lambda}^{(x)}\left(x_{1} ; x_{2}\right) & =A_{0} \cdot \exp \left[\frac{2 \pi i}{\lambda} \cdot\left(x_{1}-x_{2}\right) \cdot \sin \theta_{0}\right] \text { for }\left|x_{1}\right|,\left|x_{2}\right|<a \\
& =0 \text { otherwise }
\end{aligned}
$$

and

$$
\begin{aligned}
W_{\lambda}^{(y)}\left(y_{1} ; y_{2}\right) & =A_{0} & & \text { for }\left|y_{1}\right|,\left|y_{2}\right|<b \\
& =0 & & \text { otherwise. }
\end{aligned}
$$

These can be substituted into eq. (10.105) and its y-dependent analog. Again let us consider the spectral fluence rate in the image $p l a n e^{l}$, which is obtained by setting $x_{1}^{\prime \prime}=x_{2}^{\prime \prime}=x^{\prime \prime}$ and $y_{1}^{\prime \prime}=y_{2}^{\prime \prime}=y^{\prime \prime}$ and carrying out the integrations. The result is

${ }^{1}$ The cross spectral density function is:

$$
\begin{aligned}
& W_{\lambda}^{\prime \prime}(x)\left(x_{1}^{\prime \prime} ; x_{2}^{\prime \prime}\right)=\frac{A_{0}}{\pi^{2}} \cdot \frac{R}{R^{\prime \prime}} \cdot \exp \left[\frac{2 \pi i}{\lambda} \cdot \frac{R}{R^{\prime \prime}} \cdot\left(x_{1}^{\prime \prime}-x_{2}^{\prime \prime}\right) \cdot \sin \theta_{0}\right] \\
& \cdot\left[\operatorname{Su}\left(x_{1}^{\prime \prime}\right) \cdot \operatorname{Su}\left(x_{2}^{\prime \prime}\right)+\operatorname{Cu}\left(x_{1}^{\prime \prime}\right) \cdot \operatorname{Cu}\left(x_{2}^{\prime \prime}\right)+i \cdot \operatorname{Su}\left(x_{2}^{\prime \prime}\right) \cdot \operatorname{Cu}\left(x_{1}^{\prime \prime}\right)-i \cdot \operatorname{Su}\left(x_{1}^{\prime \prime}\right) \cdot \operatorname{Cu}\left(x_{2}^{\prime \prime}\right)\right]
\end{aligned}
$$

$W_{\lambda}^{\prime \prime}(y)\left(y_{1}^{\prime \prime} ; y_{2}^{\prime \prime}\right)$ in this example is obtained by replacing $x^{\prime \prime}, a$ and $a^{\prime}$ by $y^{\prime \prime}, b$ and $b^{\prime}$ and by setting $\theta_{0}=0$. The functions $\mathrm{Su}(\mathrm{x})$ and $\mathrm{Cu}(\mathrm{x})$ are defined in eq. (10.120). 


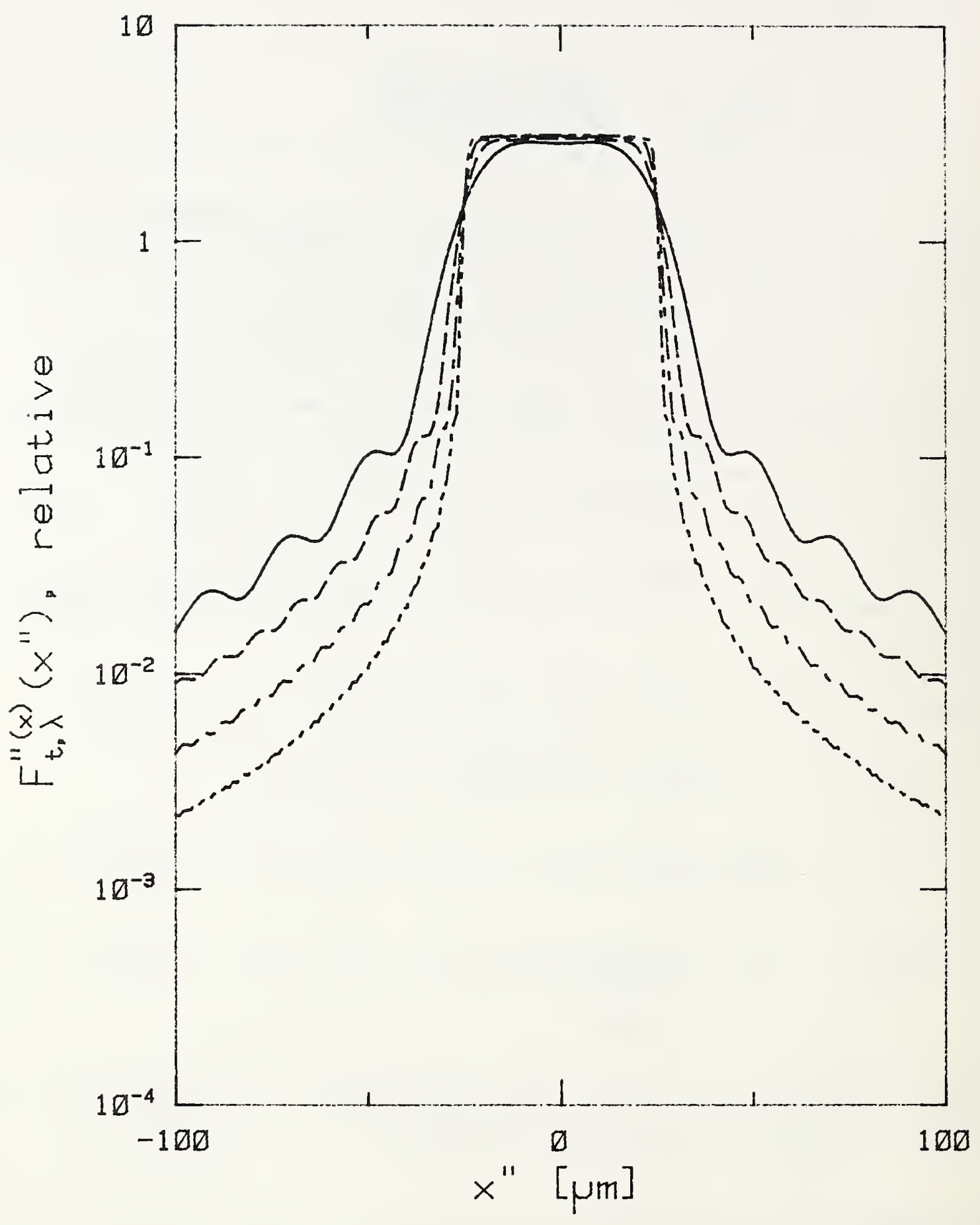

Figure 10.7. Relative distribution in the $x$-direction of fluence rate in the image of a $50[\mu \mathrm{m}]$-wide, uniform, incoherent source for various lens aperture widths. Plots of the part of eq. (10.116) within curly brackets for $\lambda=514[\mathrm{~nm}], \quad a=25[\mu \mathrm{m}], R=R^{\prime \prime}=0.8[\mathrm{~m}]$, and $-a^{\prime}=0.01[\mathrm{~m}] ;-a^{\prime}=0.02[\mathrm{~m}] ;-a^{\prime}=0.04[\mathrm{~m}]$; $--a^{\prime}=0.08[\mathrm{~m}]$. 


$$
\begin{aligned}
& F_{t, \lambda}^{\prime \prime}(x)\left(x^{\prime \prime}\right)=\frac{A_{0}}{\pi^{2}} \cdot \frac{R}{R^{\prime \prime}} \cdot\left[S^{2}\left(x^{\prime \prime}\right)+\mathrm{Cu}^{2}\left(x^{\prime \prime}\right)\right] \\
& F_{t, \lambda}^{\prime \prime(y)}\left(y^{\prime \prime}\right)=\frac{A_{o}}{\pi^{2}} \cdot \frac{R}{R^{\prime \prime}} \cdot\left\{S i\left[\frac{2 \pi b^{\prime}}{\lambda} \cdot\left(\frac{y^{\prime \prime}}{R^{\prime \prime}}+\frac{b}{R}\right)\right]-S i\left[\frac{2 \pi b^{\prime}}{\lambda} \cdot\left(\frac{y^{\prime \prime}}{R^{\prime \prime}}-\frac{b}{R}\right)\right]\right\}^{2}
\end{aligned}
$$

where

$$
\begin{aligned}
& \mathrm{Su}\left(\mathrm{x}^{\prime \prime}\right)=\frac{1}{2}\{\mathrm{Si}[\mathrm{U}(+)]-\mathrm{Si}[\mathrm{U}(-+)]+\mathrm{Si}[\mathrm{U}(+)]-\mathrm{Si}[\mathrm{U}(-)]\} \\
& \mathrm{Cu}\left(\mathrm{x}^{\prime \prime}\right)=\frac{1}{2}\{\mathrm{Ci}[\mathrm{U}(+)]-\mathrm{Ci}[\mathrm{U}(-)]-\mathrm{Ci}[\mathrm{U}(++)]+\mathrm{Ci}[\mathrm{U}(-+)]\}
\end{aligned}
$$

and

$$
\begin{aligned}
& \mathrm{U}(+)=\frac{2 \pi}{\lambda} \cdot\left(\frac{x^{\prime \prime}}{\mathrm{R}^{\prime \prime}}+\frac{a}{\mathrm{R}}\right) \cdot\left(\mathrm{a}^{\prime}+\mathrm{R} \cdot \sin \theta_{0}\right) \\
& \mathrm{U}(+)=\frac{2 \pi}{\lambda} \cdot\left(\frac{x^{\prime \prime}}{\mathrm{R}^{\prime \prime}}+\frac{\mathrm{a}}{\mathrm{R}}\right) \cdot\left(\mathrm{a}^{\prime}-\mathrm{R} \cdot \sin \theta_{0}\right) \\
& \mathrm{U}(-+)=\frac{2 \pi}{\lambda} \cdot\left(\frac{x^{\prime \prime}}{\mathrm{R}^{\prime \prime}}-\frac{\mathrm{a}}{\mathrm{R}}\right) \cdot\left(\mathrm{a}^{\prime}+\mathrm{R} \cdot \sin \theta_{0}\right) \\
& \mathrm{U}(--)=\frac{2 \pi}{\lambda} \cdot\left(\frac{x^{\prime \prime}}{\mathrm{R}^{\prime \prime}}-\frac{\mathrm{a}}{\mathrm{R}}\right) \cdot\left(\mathrm{a}^{\prime}-\mathrm{R} \cdot \sin \theta_{0}\right) .
\end{aligned}
$$

In these expressions the source has dimensions $2 a$ by $2 b$ and the aperture at the imaging optic has dimensions $2 a^{\prime}$ by $2 b^{\prime}$. The distance from the source to the aperture is $R$ and from the aperture to the image is $\mathrm{R}^{\prime \prime}$. The function $\mathrm{Ci}(\mathrm{x})$ is the cosine integral, which, for our purposes, can be defined by $\mathrm{Ci}(\mathrm{b})-\mathrm{Ci}(\mathrm{a})=\int_{\mathrm{a}}^{\mathrm{b}} \frac{\cos t}{\mathrm{t}} \cdot \mathrm{dt}$ and is tabulated in [10.15].

COHERENCE EFFECTS on a MONOCHROMATOR SLIT-SCATTERING FUNCTION. The expression for the fluence rate distribution in the image of a monochromatic rectangular coherent source [eq. (10.119) above] is of some interest because it is a model for calculating the slit-scattering function to be observed by laser irradiation of the entrance slit of a monochromator. In this application the source plane is the rectangular monochromatically irradiated entrance slit and a single image of the entrance slit is formed somewhere in the exit plane. The detector then collects the radiation passing through the exit slit as the slit is scanned across this image either by motion of the exit slit or by rotation of the dispersing element. To illustrate this calculation of a slit-scattering function we show in figure 10.8 curves of $F_{t, \lambda}(x)$ ( $)$ for several values of $\theta_{O^{\prime}}$ the angle of incidence of the laser beam on the entrance slit. The calculation gives the value of the spectral fluence rate at each point of the exit plane. This, of course, would theoretically be observable only with an infinitesimally narrow exit slit. In practice one usually has entrance and exit slits of nearly the same width so that an observed slit-scattering function would correspond to a running average (integral) of one of the curves in figure 10.8 where the width of the average would be comparable to the width of the entrance slit image -- $50 \mathrm{l \mu m}]$ in this example. The resulting smoothing would greatly reduce most of the finer structure of the curves. But it would not eliminate the high level of the far wings of the slit-scattering function when the angle of incidence corresponds to pointing the laser at the edge of the imaging aperture (the edge of the grating, $\theta_{0}=0.047$ [rad] in figure 10.8) or the splitting of the slit-scattering function into twin peaks at larger angles of incidence. The values of the parameters chosen for this example correspond to those of a moderately high resolution single monochromator (monochromator focal length $R=R^{\prime \prime}=0.8[\mathrm{~m}]$, slit halfwidth $a=25[\mu \mathrm{m}]$, grating projected halfwidth a'=0.0375 [m], and wavelength $\lambda=514$ [nm].) 


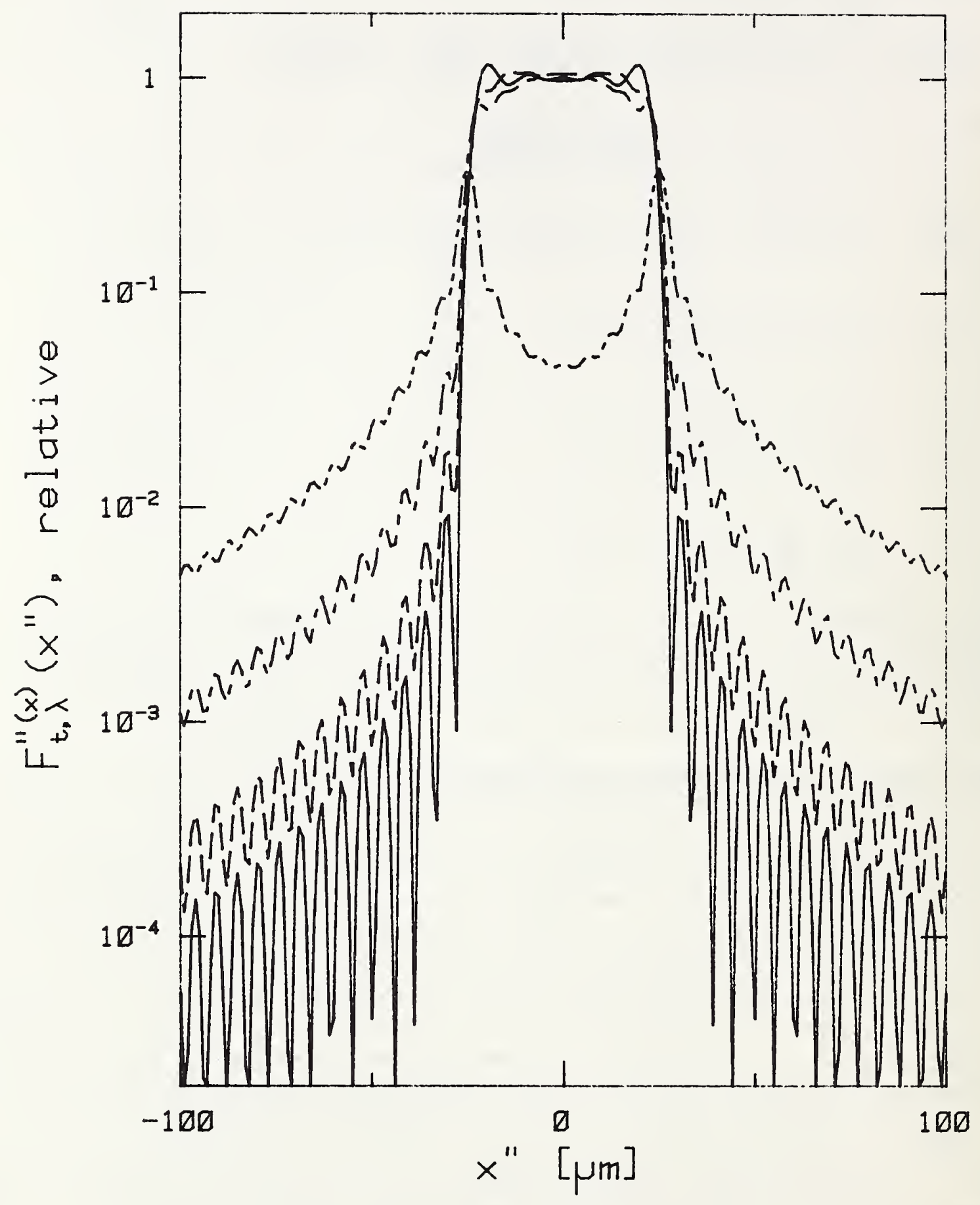

Figure 10.8. Relative distribution in the $x$-direction of fluence rate in the image of a 50 [ $\mathrm{mm}]$-wide, uniform, coherent source for various angles of incidence of the beam. Plots of the double integral of eq. (10.119) for the same parameter values as in figure 10.7 except $a^{\prime}=0.0375[\mathrm{~m}]$ and $A_{0}=1 .-\theta_{0}=0.00$ [rad]; $-\theta_{0}=0.03[\mathrm{rad}] ;-\theta_{0}=0.04[\mathrm{rad}] ;--\theta_{0}=0.05[\mathrm{rad}]$. 
These curves show that the measurement of the slit-scattering function with a laser beam requires careful attention to the angle of incidence. With incoherent light this problem doesn't arise because there is no particular direction associated with incoherent light aside from the obvious dependence upon the projected size of the source. This point is illustrated if we imagine trying to produce incoherent light in the entrance slit of a monochromator. This can be done by replacing the slit by an incandescent filament or by illuminating the slit by an infinite incoherent source (an integrating sphere with one port being the entrance slit would probably do). In neither case is there any defined direction of the sort exhibited by the laser.

So far we have limited our examples to the two extreme cases of complete incoherence and complete coherence. The reason for this is that these are the two cases for which the integrations are most easily carried out -- incoherence, because of the delta function -- and coherence, because of the factorization into the product of a simpler integral and its complex conjugate. The theory, however, is perfectly general and applies to any expression for the cross-spectral density with any degree of coherence.

As a final numerical example of the application of the propagation of coherence to practical radiometry we will calculate for partially coherent radiation the slit-scattering function of the monochromator of the last example (see also reference [10.18]). We will assume that the entrance slit is irradiated by a monochromatic incoherent source of width $2 a_{0}$ located on the optic axis at a distance $R_{0}$ in front of the slit and will calculate the distribution of spectral fluence rate in the exit plane. As we have seen [eq. (10.52)] the $x-$ dependent part of the degree of coherence of the light at the plane of the entrance slit depends upon the ratio $a_{0} / R_{0}$ and can be varied from total coherence to total incoherence by changing this ratio from 0 to $\infty$. Based upon eq. (10.47), we see that we can write the $x-$ dependent factor of the cross-spectral density function in the plane of the entrance slit as

$$
w_{\lambda}^{(x)}\left(x_{i} ; x_{2}\right)=\frac{2 a_{0} A_{0}}{R_{0}} \cdot \operatorname{sinc}\left[\frac{2 a_{0}\left(x_{1}-x_{2}\right)}{\lambda R_{0}}\right] \text {. }
$$

Now, using eq. (10.105), we obtain for the cross spectral density in the plane of the exit slit:

$$
\begin{aligned}
w_{\lambda}^{\prime \prime}(x)\left(x_{1}^{\prime \prime} ; x_{2}^{\prime \prime}\right)= & \frac{8 a^{\prime 2} a_{0} A_{0}}{R_{0} R R^{\prime \prime} \lambda^{2}} \cdot \int_{-a}^{a} \int_{-a}^{a} \operatorname{sinc}\left[\frac{2 a_{0}\left(x_{1}-x_{2}\right)}{\lambda R_{0}}\right] \\
& \cdot \operatorname{sinc}\left[\frac{2 a^{\prime}}{\lambda} \cdot\left(\frac{x_{1}}{R}+\frac{x_{1}^{\prime \prime}}{R^{\prime \prime}}\right)\right] \cdot \operatorname{sinc}\left[\frac{2 a^{\prime}}{\lambda} \cdot\left(\frac{x_{2}}{R}+\frac{x_{2}^{\prime \prime}}{R^{\prime \prime}}\right)\right] \cdot d x_{1} \cdot d x_{2} \cdot
\end{aligned}
$$

In this expression $2 a^{\prime}$ is the width of the instrument aperture -- usually this is the width of the grating -- and $R$ and $R^{\prime \prime}$ are the distances from the entrance and exit slits to the collimating optics. If we confine our attention to the spectral fluence rate in the exit plane we can set $x_{2}^{\prime \prime}=x_{1}^{\prime \prime}=x^{\prime \prime}$ to give the $x$-component as

$$
\begin{aligned}
& F_{t, \lambda}^{\prime \prime}(x)=\frac{8 a^{\prime 2} a_{0} A_{0}}{R_{0} R R^{\prime \prime} \lambda^{2}} \cdot \int_{-a}^{a} \int_{-a}^{a} \operatorname{sinc}\left[\frac{2 a_{0}}{\lambda R_{0}} \cdot\left(x_{1}-x_{2}\right)\right] \\
& \cdot \operatorname{sinc}\left[\frac{2 a^{\prime}}{\lambda} \cdot\left(\frac{x_{1}}{R}+\frac{x^{\prime \prime}}{R^{\prime \prime}}\right)\right] \cdot \operatorname{sinc}\left[\frac{2 a^{\prime}}{\lambda} \cdot\left(\frac{x_{2}}{R}+\frac{x^{\prime \prime}}{R^{\prime \prime}}\right)\right] \cdot d x_{1} \cdot d x_{2} \cdot
\end{aligned}
$$


The results of performing the indicated integrations numerically for the monochromator of the previous example $\left(R=R^{\prime \prime}=0.8[\mathrm{~m}], \mathrm{a}^{\prime}=0.0375[\mathrm{~m}], \quad \mathrm{a}=25[\mu \mathrm{m}], \lambda=514[\mathrm{~nm}]\right)$ are shown in figure 10.9. The two solid curves are the extremes of complete coherence and complete incoherence; the incoherent curve possesses the highest slit-scattering function in the far wings and is quite flat and structureless in the middle. The dashed curves show cases of partial coherence and lie generally between the two extremes. The curves in this figure have been area normalized in order to emphasize the changing shape of the slit-scattering function. Again the figure shows the distribution of fluence rate in the exit plane of the monochromator. This would be observable with a very narrow exit slit. For a normal exit slit the finer structure would be largely smoothed out and only the average far-wing behavior would distinguish the cases of differing coherence.

Clearly, if the slit-scattering function of a spectroradiometer must be measured accurately for later use (deconvolution) with an unknown source it is necessary that the state of coherence of the calibration beam be similar to that of the unknown to be measured. For many situations this requirement may not be as restrictive as it appears. In figure 10.9 , for example, the transition from slit-scattering functions which are barely distinguishable from completely coherent to those which are barely distinguishable from completely incoherent occurs as $a_{0} / R_{0}$ changes from about 0.03 to 0.06 . Thus many radiation fields could probably be treated as one extreme or the other. In addition, by changing some parameter of the measurement environment such as $\mathrm{R}_{\mathrm{O}}$, the source-radiometer distance, it may be possible to shift the slit-scattering functions for both sources to the same side of the transition region, so that for that particular pair of measurements, at least, a common slit-scattering function will be satisfactory.

More generally, we can say that for an imaging system, if the coherence interval in the object plane is very much greater than the width of the point spread function of the optical system in the object plane, then the image will resemble the coherent limit. If the coherence interval is very much smaller than the width of the point spread function then the image will resemble the incoherent limit. The point spread function of an imaging system is the distribution of fluence rate in the image of a point source. Ideally a point source should be imaged into a point image but due to diffraction, aberrations, and focusing errors it never will be. Here we are only interested in the scattering by diffraction. For perfect imagery and rectangular apertures we can calculate the point spread function from eq. (10.105) Imagine the source shrinking to a very small area around $x_{1}=x_{2}=0$. The source crossspectral density $\mathrm{w}_{\lambda}^{(\mathrm{x})}\left(\mathrm{x}_{1} ; \mathrm{x}_{2}\right)$ will then approach a constant, $F_{t, \lambda}(\mathrm{x})(0)$. Thus, the $\mathrm{x}$-dependent factor of the image cross-spectral density function will be proportional to:

$$
\operatorname{sinc}\left(2 a^{\prime} x_{1}^{\prime \prime} / \lambda R^{\prime \prime}\right) \cdot \operatorname{sinc}\left(2 a^{\prime} x_{2}^{\prime \prime} / \lambda R^{\prime \prime}\right)
$$

and the fluence rate in the image will be proportional to $\operatorname{sinc}^{2}\left(2 a^{\prime} x^{\prime \prime} / \lambda R^{\prime \prime}\right)$. There is, of course, a similar $y$-dependent factor and the complete point spread function for a rectangular aperture contains the product of these two factors. In one dimension the point spread function is just the slit-scattering function for an infinitesimal slit. To obtain the point spread function in the object plane we interchange the roles of image and object and write the point spread function as proportional to $\operatorname{sinc}^{2}\left(2 a^{\prime} x / \lambda R\right)$. The width of this function is then, say, twice the value of $x$ at which the function is reduced to half its peak value. Actually, with sinc functions it is more common to use the width between zeros of the function, but to be consistent with our choice for coherence interval [eq. (10.56)] we will continue to use the full width at half height. Since $\operatorname{sinc}^{2}(u)=0.5$ for $u=0.44$, we find that the width of the point spread function is about $0.4 \lambda \mathrm{R} / \mathrm{a}^{\prime}$. This can also be interpreted as the resolution limit of the imaging system; two objects lying closer together than this will not 


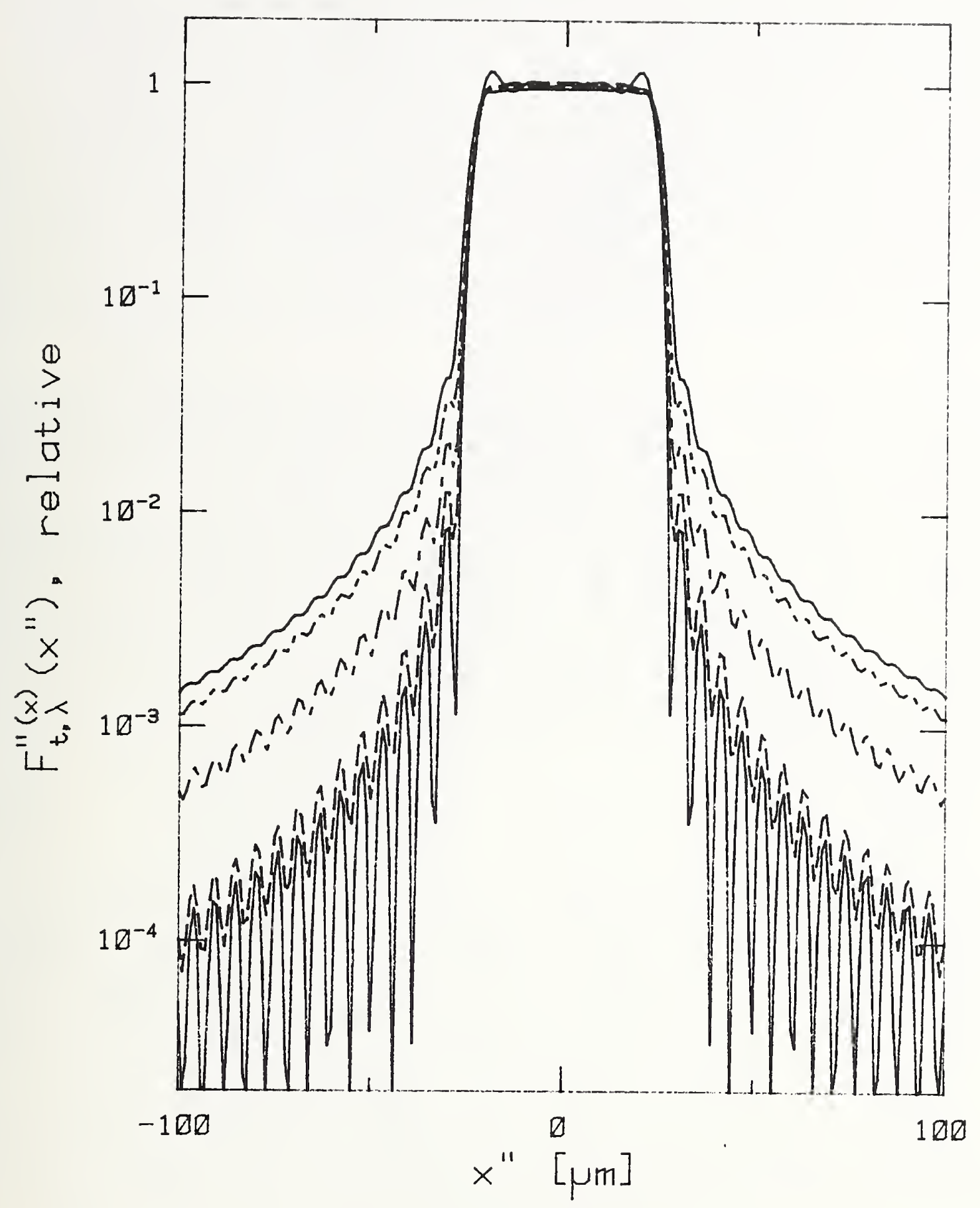

Figure 10.9. Relative distribution in the $x$-direction of fluence rate

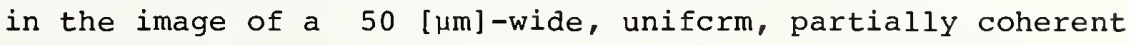
source at normal incidence for various degrees of partial coherence. Plots of the double integral of eq. (10.124) for the same parameter values as in figure 10.8 and area normalized, in the interval shown, to the area under the $\theta_{0}=0$ curve in figure 10.8. $a_{0} / R_{0}=0.00$ (coherent); $\longrightarrow a_{0} / R_{0}=0.04 ;-a_{0} / R_{0}=0.045 ;--a_{0} / R_{0}=0.05$ $-a_{0} / R_{0}=\infty$ (incoherent). 
be resolved in the image. For our present purpose of considering the effects of coherence in radiometry, the significance of the point spread function width is that if, for two different sources, the characteristic coherence intervals at the entrance slit of a monochromator radiometer are either both very much greater than $\lambda R / a^{\prime}$ or both very much smaller than $\lambda \mathrm{R} / \mathrm{a}^{\prime}$ then the monochromator slit-scattering function will be similar for both sources. If one or both coherence intervals are comparable to $\lambda \mathrm{R} / \mathrm{a}^{\prime}$ or, worse, if one is large and the other small, then the slit-scattering functions may differ considerably for the two sources, and, depending upon their spectral distributions, this may significantly affect the accuracy of any direct comparison of the two sources with this radiometer. To test if this is a problem or to ameliorate the problem if it exists one can usually increase the point spread function width by decreasing the imaging aperture halfwidth a' or, as mentioned above, one may be able to change the coherence interval by changing the distance from the source to the slit.

Any time high resolution is sought in any dimension of the measurement situation -spectral, temporal, angular, or spatial -- the coherence state of the radiation can affect the measurement results. In the present example spectral resolution is achieved by means of spatial and angular resolution of an image within the monochromator. This image resolution is limited by the distortion caused by diffraction, and the magnitude and nature of the distortion due to diffraction are dependent upon the coherence state of the radiation entering the radiometer. As a further example let us consider the image formed by a lens of a slit upon which a laser speckle pattern falls. If we assume that the speckles consist of homogeneous regions all of the same size but with random electromagnetic field amplitudes and phases then the fluence rate in the image may be distributed somewhat like one of the curves in figure 10.10. The curves in this figure were computed for the same monochromator as in the previous examples, but with the slit coherently irradiated with a cross-spectral density given by:

$$
\mathrm{w}_{\lambda}{ }^{(\mathrm{x})}\left(\mathrm{x}_{1} ; \mathrm{x}_{2}\right)=A \cdot \cos \left[\alpha\left(\mathrm{x}_{1}\right)\right] \cdot \cos \left[\alpha\left(\mathrm{x}_{2}\right)\right] \cdot \exp \left[\mathrm{i} \beta\left(\mathrm{x}_{1}\right)-i \beta\left(\mathrm{x}_{2}\right)\right]
$$

where $\alpha(x)$ and $\beta(x)$ are angles between $-\pi$ and $\pi$ which are constant within each interval of $1[\mu \mathrm{m}]$ but random from interval to interval across the width of the slit. The two curves are for two different samples of random $\alpha(x)$ and $\beta(x)$ values. Now suppose that the speckle pattern moves with time (e.g., is generated by a rotating ground glass) or, equivalently, suppose that the primary source is polychromatic in such a way that the phase in each speckle changes in time randomly with respect to its neighbors. Then the distribution of fluence rate in the image will evolve continuously from curve to curve among the family of curves of which those of figure 10.10 are representative. If a small detector in the image plane possesses sufficient temporal resolution and if the speckle pattern evolves sufficiently slowly then the detector will follow this evolution and an erratic signal output will result. On the other hand, if the detector in the image plane has poor temporal resolution and if the speckle pattern changes rapidly then a steady average signal output will result and, in fact, the average distribution of fluence rate in the image will be exactly that of an incoherently irradiated slit: the average of a large number of random speckle pattern image distributions such as those of figure 10.10 approaches the shape of the incoherent curve of figure 10.9. Thus, a rapidly changing, fine, speckle pattern is a model for incoherent light and we see that with high temporal resolution the results of a single radiometric measurement can depend upon the coherence state of the radiation. Although in this example we have employed the time averaging of an evolving speckle pattern to simulate 'natural' incoherent light, the average in the y-direction -- along the height of the slit -of a stationary speckle pattern can produce the same effect. We have assumed, in the 


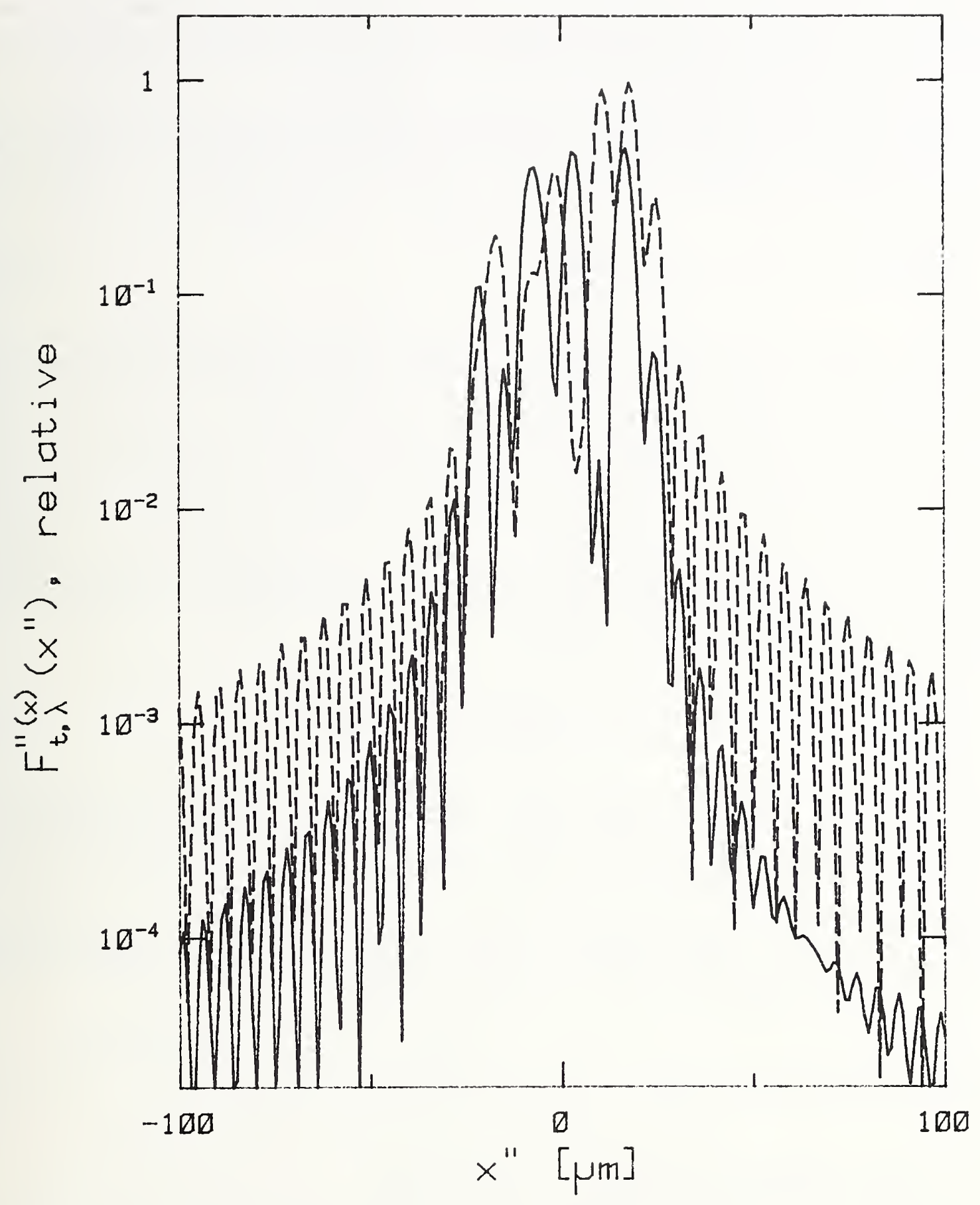

Figure 10.10. Two samples of the relative distribution in the $x$-direction

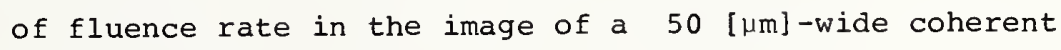
source consisting of 1 [ $\mu \mathrm{m}]$ cells with amplitude and phase constant within each cell but random from cell to cell. 
calculations of figure 10.10, that the height of the slit spans one speckle width or less and have ignored the $y$-dimension of the problem. If the slit and detector cover many speckles in the vertical direction then again the detector will integrate over many random speckle patterns in this direction and the observed fluence rate distribution in the image plane will agree with that of an incoherently irradiated slit even when the speckle pattern is stationary and the irradiation is monochromatic.

MORE on IMAGING -- PUPIL FUNCTIONS and ABERRATIONS. In deriving eq. (10.98) for the crossspectral density function in the image of a source we made two applications of eq. (10.36) -the law of propagation of cross-spectral density. This gives, among other factors in the integrand, a phase factor

$$
\exp \left[\frac{2 \pi i}{\lambda} \cdot\left(s_{1}+s_{1} "-s_{2}-s_{2} "\right)\right]
$$

The distance $s_{1}+s_{1} "$ is the total optical path length from point $\left(x_{1}, y_{1}\right)$ in the source plane to point $\left(x_{1}^{\prime}, y_{1}^{\prime}\right)$ in the image plane via a point $\left(x_{1}^{\prime}, y_{1}^{\prime}\right)$ in the intermediate aperture plane. Similarly $s_{2}+s_{2}^{\prime \prime}$ is the total optical path length between a second pair of source and image points via a second point in the aperture. In that derivation we made some approximations for this phase factor and then, in passing to eq. (10.99), we integrated over all pairs of points in the aperture. It is not necessary, of course, that there be any focusing optic in the aperture plane or that there be a recognizable image; the "source", "aperture", and "image" planes may be any three consecutive cross-sections of the beam and the sequential application of eq. $(10.36)$ is still valid. The particular simplifications which led to eq. (10.98) will no longer apply in the absence of perfect imagery. Instead, the second fourfold integral in eq. (10.98) will be replaced by

$$
\iiint \int_{\text {aperture }} \exp \left[\frac{2 \pi i}{\lambda} \cdot\left(s_{1}+s_{1}^{\prime \prime}-s_{2}-s_{2}^{\prime \prime}\right)\right] \cdot d x_{1}^{\prime} \cdot d y_{1}^{\prime} \cdot d x_{2}^{\prime} \cdot d y_{2}^{\prime}
$$

where the integrations cover the aperture twice. Starting from expression (10.128) we now want to discuss a generalization of eq. (10.98) which can include arbitrary apertures and departures from perfect imagery.

First of all, instead of limiting the integrations to the aperture we can introduce a pupil function ${ }^{1} P\left(x^{\prime}, y^{\prime}\right)$ whose value is 1 inside the aperture and 0 outside the aperture and then let the integrations cover the entire aperture plane:

$$
\int_{-\infty}^{\infty} \int_{-\infty}^{\infty} \int_{-\infty} P\left(x_{1}^{\prime}, y_{1}^{\prime}\right) \cdot P *\left(x_{2}^{\prime}, y_{2}^{\prime}\right) \cdot \exp \left[\frac{2 \pi i}{\lambda} \cdot\left(s_{1}+s_{1}^{\prime \prime}-s_{2}-s_{2}^{\prime \prime}\right)\right] \cdot d x_{1}^{\prime} \cdot d y_{1}^{\prime} \cdot d x_{2}^{\prime} \cdot d y_{2}^{\prime} \cdot
$$

For our rectangular aperture, for example, we would have

$$
\begin{aligned}
P\left(x^{\prime}, y^{\prime}\right) & =1 \text { for }-a<x^{\prime}<a \text { and }-b<y^{\prime}<b \\
& =0 \text { otherwise. }
\end{aligned}
$$

\footnotetext{
${ }^{1}$ Strictly speaking the pupil function $P\left(x^{\prime}, y^{\prime}\right)$ will also depend upon the source point $(x, y)$ and the destination point $\left(x^{\prime \prime}, y^{\prime \prime}\right)$ of the radiation passing through the aperture. This dependence is generally neglected and we don't explicitly show it in our notation.
} 
So far this is exactly equivalent to expression (10.128). However, we can generalize this expression by allowing $P\left(x^{\prime}, y^{\prime}\right)$ to take on values other than 0 and 1 . Intermediate values of $P\left(x^{\prime}, y^{\prime}\right)$ can describe optical systems involving partially absorbing media such as the "aperture" formed by the slide in a slide projector. If the aperture introduces phase variations, such as a slide made of wavy glass, then this can either be put into the pupil function by making $P\left(x^{\prime}, y^{\prime}\right)$ complex or be accounted for in the calculation of $s+s^{\prime \prime}$.

The four-fold integral in expression (10.129) can be seen to be the product of two similar integrals:

$$
\begin{aligned}
& \iint_{-\infty}^{\infty} P\left(x_{1}^{\prime}, y_{1}^{\prime}\right) \cdot \exp \left[\frac{2 \pi i}{\lambda} \cdot\left(s_{1}+s_{1}^{\prime \prime}\right)\right] \cdot d x_{1}^{\prime} \cdot d y_{i}^{\prime} \\
& \cdot \iint_{-\infty}^{\infty} P *\left(x_{2}^{\prime}, y_{2}^{\prime}\right) \cdot \exp \left[-\frac{2 \pi i}{\lambda} \cdot\left(s_{2}+s_{2}^{\prime \prime}\right) \cdot d x_{2}^{\prime} \cdot d y_{2}^{\prime}\right] \\
& =K\left(x_{1}, y_{1} ; x_{1}^{\prime \prime}, y_{1}^{\prime \prime}\right) \cdot K^{*}\left(x_{2}, y_{2} ; x_{2}^{\prime \prime}, y_{2}^{\prime \prime}\right),
\end{aligned}
$$

where

$$
K\left(x, y ; x^{\prime \prime}, y^{\prime \prime}\right)=\iint_{-\infty}^{\infty} P\left(x^{\prime}, y^{\prime}\right) \cdot \exp \left[\frac{2 \pi i}{\lambda} \cdot\left(s+s^{\prime \prime}-R-R^{\prime \prime}\right)\right] \cdot d x^{\prime} \cdot d y^{\prime}
$$

and $s+s^{\prime \prime}$ is the optical path length between ( $x, y$ ) and ( $\left.x^{\prime \prime}, y^{\prime \prime}\right)$ via ( $\left.x^{\prime}, y^{\prime}\right)$. $R$ is the perpendicular optical path length from the source plane to the aperture plane and $R^{\prime \prime}$ that from the aperture plane to the image plane. The exponential factors involving $R$ and $R^{\prime \prime}$ which we have introduced in eq. (10.132) cancel out in forming eq. (10.131). The function K( $\left.x, y ; x^{\prime \prime}, y^{\prime \prime}\right)$ is the transmission function [10.2], [10.3], and [10.5] of the system. The transmission function is similar to those functions variously known as point-spread function, impulse function, Green's function, etc.: if we think of $\mathrm{K}\left(\mathrm{x}, \mathrm{y} ; \mathrm{x}^{\prime \prime} \mathrm{Y}^{\prime \prime}\right)$ as a function of the position of the point ( $\mathrm{x}^{\prime \prime}, \mathrm{y}^{\prime)}$ in the image plane, then it describes the field in the image plane caused by a unit point source of fixed phase at the source point $(x, y)$. In terms of the transmission function we can now write the cross-spectral density function in the image plane as

$$
\begin{aligned}
& W_{\lambda}^{\prime \prime}\left(x_{1}^{\prime \prime}, y_{1}^{\prime \prime} ; x_{2}^{\prime \prime}, y_{2}^{\prime \prime}\right)=\frac{1}{R^{2} R^{\prime \prime}{ }_{\lambda}^{4}} \cdot \iiint \int_{\text {Source }} w_{\lambda}\left(x_{1}, y_{1} ; x_{2}, y_{2}\right) \\
& \cdot K_{\lambda}\left(x_{1}, y_{1} ; x_{l}^{\prime \prime}, y_{1}^{\prime \prime}\right) \cdot K_{\lambda}^{\star}\left(x_{2}, y_{2} ; x_{2}^{\prime \prime}, y_{2}^{\prime \prime}\right) \cdot d x_{1} \cdot d y_{1} \cdot d x_{2} \cdot d y_{2} \cdot
\end{aligned}
$$

This is the generalization of eq. (10.98) which applies whatever the aperture and whether or not imagery is good. Of course, it requires being able to express the optical path lengths $s+s^{\prime \prime}$ in terms of the coordinates of the source, aperture, and image points.

When the aberrations or focusing errors are small we can write

$$
s+s^{\prime \prime}=R-\frac{x x^{\prime}+\underline{y} \underline{y}^{\prime}}{R}+R^{\prime \prime}-\frac{x^{\prime} x^{\prime \prime}+y^{\prime} y^{\prime \prime}}{R^{\prime \prime}}+\Delta
$$

where the first four terms on the right come from the approximations which lead to eq. (10.98) [see also eq. (10.39)] and $\Delta$ is a correction term. Notice that if $\Delta$ is zero 
there exists a point in the image plane at $x^{\prime \prime}=-x \cdot R^{\prime \prime} / R, y^{\prime \prime}=-y \cdot R^{\prime \prime} / R$ for which $s+s^{\prime \prime}$ is independent of the aperture point $\left(x^{\prime}, y^{\prime}\right)$. This means that for each source point ( $x, y$ ) there is a conjugate image point for which all rays through the aperture travel the same optical path length. Thus, eq. (10.134) with $\Delta=0$, and consequently also eqs. (10.98) and (10.99) correspond to perfect, aberration-free focusing. ${ }^{1}$

Equation (10.133) with the transmission function defined by eq. (10.132) is always valid provided only that the optical path lengths $s_{1}$ and $s_{2}$ (and $s_{1}^{\prime \prime}$ and $s_{2}^{\prime \prime}$ which appear in the denominator of the propagation law [eq. (10.36)] are sufficiently constant to be approximated by $R$ (and $R^{\prime \prime}$ ). ${ }^{2}$ If the departures from perfect imagery are small the approximation of eq. (10.134) may be useful. As an example of this approximation suppose that although the true focal plane is located at the distance R" from the aperture the "image" plane of interest is a parallel plane a small distance $\zeta$ beyond the focal plane. Then a first approximation to $\Delta$ is given [10.2] by

$$
\Delta=\zeta \cdot\left(1-\frac{x^{\prime 2}+y^{\prime 2}}{2 R^{\prime 2}}\right)
$$

Focus errors are treated in full in reference [10.2], Chapter 8, and aberrations are treated in Chapter 9 of the same reference. It will rarely be possible to evaluate in terms of convenient, known functions the integrals which result from the inclusion of focus errors, aberrations or exotic pupil functions. Usually, the numerical evaluation of the transmission functions from eq. (10.132) with $s_{1}, s_{1}^{\prime}, s_{2}$ and $s_{2}^{\prime \prime}$ calculated directly, will be preferable to trying to identify and work with the terms of the expansion of eq. (10.134). By writing complex exponentials as the sum of a cosine term and $i$ times a sine term, the integrals of eqs. (10.132) and (10.133) can ultimately be expressed as a sum of two integrals -one real and one imaginary -- which can be computed independently. Eventually, in the chain of calculations when an observable such as fluence rate or radiant intensity is computed, these components will combine to produce a single, real, result.

${ }^{1}$ In this case [or if we put any phase error factor, exp $\frac{2 \pi i \Delta}{\lambda}$, into the pupil function $\left.P\left(x^{\prime}, y^{\prime}\right)\right]$ we can write the transmission function as

$$
K\left(x, y ; x^{\prime \prime}, y^{\prime \prime}\right)=\int_{-\infty}^{\infty} P\left(x^{\prime}, y^{\prime}\right) \cdot \exp \left[-\frac{2 \pi i}{\lambda} \cdot\left(\frac{x x^{\prime}+y y^{\prime}}{R}+\frac{x^{\prime} x^{\prime \prime}+y^{\prime} y^{\prime \prime}}{R^{\prime \prime}}\right)\right] \cdot d x^{\prime} \cdot d y^{\prime}
$$

Now by letting $\xi=x / R+x^{\prime \prime} / R^{\prime \prime}$ and $\eta=y / R+y^{\prime \prime} / R^{\prime \prime}$ this integral can be cast in the form of a two-dimensional Fourier transform of the pupil function (as modified by including any phase factors for aberrations or focus error):

$$
K\left(x, y ; x^{\prime \prime}, y^{\prime \prime}\right)=K(\xi, n)=\iint_{-\infty}^{\infty} P\left(x^{\prime}, y^{\prime}\right) \cdot \exp \left[-\frac{2 \pi i}{\lambda} \cdot\left(x^{\prime} \xi+y^{\prime} n\right)\right] \cdot d x^{\prime} \cdot d y^{\prime} \cdot
$$

Many of the equations of this chapter can be expressed somewhat more compactly by employing Fourier transform shorthand. However, we make no use of this because we suspect that many readers never really feel comfortable with Fourier transforms and we fear that their use sometimes tends to obscure the simple physics and the essential approximations involved.

${ }^{2} \mathrm{All}$ lengths, including $\mathrm{R}$ (and $\mathrm{R}^{\prime \prime}$ ), must of course be $>\lambda$. 
The CLASSICAL RADIOMETRIC QUANTITIES. So far in this chapter we have avoided saying much about spectral radiance and this, no doubt, seems curious since spectral radiance lies at the heart of all the rest of the chapters of this Manual. The reason is that the classical concept of spectral radiance is inconsistent with a wave theory of light because the very limiting process required to define a ray and, hence, radiance (letting the areas of two apertures go to zero -- see Chapter 2 [10.19]) guarantees such severe diffraction in the presence of coherence that the direction the "ray" came from is totally indeterminate. In consequence, no function of the cross-spectral density can possess all the properties of the classically defined spectral radiance for all fields [10.14]. Functions which possess many of the properties of spectral radiance can be defined and that given in eq. (10.20) is commonly used.

Equation (10.20) can be inferred from eq. (10.36) for the propagation of the cross-spectral density function using the classical relationship between spectral radiance and spectral fluence rate. Consider a source plane $z=0$ where the spectral radiance is desired. In this plane we assume that the cross-spectral density $W_{\lambda}\left(x_{1}, y_{1} ; x_{2}, y_{2}\right)$ is known. To measure the spectral radiance we would place a spectroradiometer at some distance $\mathrm{R}$ away from the source, pointing from the direction $\theta, \phi$ to the point $x, y$ where the spectral radiance is desired. At the entrance aperture of the radiometer the cross-spectral density is given by eq. $(10.36)$. Let us express eq. (10.36) in terms of the average and difference coordinates introduced in eqs. (10.77) and (10.82):

$$
\begin{array}{ll}
x=\frac{1}{2}\left(x_{1}+x_{2}\right) & y=\frac{1}{2}\left(y_{1}+y_{2}\right) \\
\xi=x_{1}-x_{2} & \eta=y_{1}-y_{2} .
\end{array}
$$

Then $x_{1}=x+\frac{1}{2} \xi, y_{1}=y+\frac{1}{2} \eta, x_{2}=x-\frac{1}{2} \xi, y_{2}=y-\frac{1}{2} \eta$, and the product of the area elements becomes ${ }^{1} \mathrm{dx} \cdot \mathrm{d} y \cdot \mathrm{d} \xi \cdot \mathrm{d} \eta$. We also make similar substitutions for the primed coordinates in the $X^{\prime}-Y^{\prime}$ plane and define $R$ as the distance between the source point $P(x, Y, 0)$ and the distant point $P^{\prime}\left(x^{\prime}, Y^{\prime}, z^{\prime}\right)$ where the radiometer is located:

$$
\mathrm{R}^{2}=\left(\mathrm{x}^{\prime}-\mathrm{x}\right)^{2}+\left(\mathrm{y}^{\prime}-\mathrm{y}\right)^{2}+\mathrm{z}^{\prime 2}
$$

In terms of these variables the distances $s_{1}$ and $s_{2}$ of eq. (10.36) become

$$
\begin{aligned}
& s_{1}=\sqrt{R^{2}+\left(x^{\prime}-x\right) \cdot\left(\xi^{\prime}-\xi\right)+\left(y^{\prime}-y\right) \cdot\left(n^{\prime}-\eta\right)+\frac{1}{4}\left[\left(\xi^{\prime}-\xi\right)^{2}+\left(\eta^{\prime}-\eta\right)^{2}\right]} \\
& s_{2}=\sqrt{R^{2}-\left(x^{\prime}-x\right) \cdot\left(\xi^{\prime}-\xi\right)-\left(y^{\prime}-y\right) \cdot\left(n^{\prime}-\eta\right)+\frac{1}{4}\left[\left(\xi^{\prime}-\xi\right)^{2}+\left(\eta^{\prime}-\eta\right)^{2}\right]}
\end{aligned}
$$

and we can write eq. $(10.36)$ as

$$
\begin{aligned}
w_{\lambda}^{\prime}\left(x^{\prime}+\frac{1}{2} \xi^{\prime}, y^{\prime}+\frac{1}{2} \eta^{\prime} ; x^{\prime}-\frac{1}{2} \xi^{\prime}, y^{\prime}-\frac{1}{2} \eta^{\prime}\right) & =\iiint \int_{\text {source }} w_{\lambda}\left(x+\frac{1}{2} \xi, y+\frac{1}{2} \eta ; x-\frac{1}{2} \xi, y-\frac{1}{2} \eta\right) \\
& \cdot \exp \left[\frac{2 \pi i}{\lambda} \cdot\left(s_{1}-s_{2}\right)\right] \cdot \frac{z^{\prime}{ }^{2}}{s_{1}{ }^{2} s_{2}{ }^{2} \lambda^{2}} \cdot d x \cdot d y \cdot d \xi \cdot d \eta
\end{aligned}
$$

\footnotetext{
${ }^{1}$ See footnote on page 27.
} 
Now we make the usual approximations; we expand the square roots in eqs. (10.140) in powers of $1 / R$ as in going from eq. (10.38) to eq. (10.41), keeping only the lowest order terms to give

$$
\begin{aligned}
& w_{\lambda}^{\prime}\left(x^{\prime}+\frac{1}{2} \xi^{\prime}, y^{\prime}+\frac{1}{2} \eta^{\prime} ; x^{\prime}-\frac{1}{2} \xi^{\prime}, y^{\prime}-\frac{1}{2} \eta^{\prime}\right)=\iiint \int_{\text {source }} w_{\lambda}\left(x+\frac{1}{2} \xi, y+\frac{1}{2} \eta ; x-\frac{1}{2} \xi, y-\frac{1}{2} \eta\right) \\
& \quad \exp \left\{\frac{2 \pi i}{\lambda R} \cdot\left[\left(x^{\prime}-x\right) \cdot\left(\xi^{\prime}-\xi\right)+\left(y^{\prime}-y\right) \cdot\left(\eta^{\prime}-\eta\right)\right]\right\} \cdot \frac{z^{\prime 2}}{R^{4} \lambda^{2}} \cdot d x \cdot d y \cdot d \xi \cdot d \eta \cdot
\end{aligned}
$$

The spectral fluence rate at $P^{\prime}\left(x^{\prime}, y^{\prime}, z^{\prime}\right)$ is obtained by setting $\xi^{\prime}=\eta^{\prime}=0$ :

$$
\begin{aligned}
& F_{t, \lambda}^{\prime}\left(x^{\prime}, y^{\prime}\right)=\iiint \int_{\text {source }} w_{\lambda}\left(x+\frac{1}{2} \xi, y+\frac{1}{2} \eta ; x-\frac{1}{2} \xi, y-\frac{1}{2} \eta\right) \\
& \cdot \exp \left\{-\frac{2 \pi i}{\lambda R} \cdot\left[\xi \cdot\left(x^{\prime}-x\right)+n \cdot\left(y^{\prime}-y\right)\right]\right\} \cdot \frac{z^{\prime 2}}{R^{4} \lambda^{2}} \cdot d x \cdot d y \cdot d \xi \cdot d \eta \cdot
\end{aligned}
$$

The contribution to the fluence rate from the element of solid angle $d \omega=\frac{\cos \theta}{R^{2}} \cdot d x \cdot d y$ is just

$$
\begin{aligned}
& \mathrm{dF}_{t, \lambda}^{\prime}\left(\mathrm{x}^{\prime}, \mathrm{y}^{\prime}, \theta, \phi\right)=\iint_{\text {source }} \mathrm{w}_{\lambda}\left(\mathrm{x}+\frac{1}{2} \xi, y+\frac{1}{2} \eta ; \mathrm{x}-\frac{1}{2} \xi, \mathrm{y}-\frac{1}{2} \eta\right) \\
& \cdot \exp \left\{-\frac{2 \pi i}{\lambda R} \cdot\left[\xi \cdot\left(x^{\prime}-x\right)+\eta \cdot\left(y^{\prime}-y\right)\right]\right\} \cdot \frac{z^{\prime}}{\mathrm{R} \lambda^{2}} \cdot d \xi \cdot d \eta \cdot d \omega \cdot
\end{aligned}
$$

Therefore, from the classical relation $\{\text { eq. (4.19) [10.9] }\}^{1}$

$$
L^{\prime}\left(x^{\prime}, y^{\prime}, \theta, \phi\right)=\frac{d F_{t}^{\prime}\left(x^{\prime}, Y^{\prime}, \theta, \phi\right)}{d \omega}
$$

we obtain

\footnotetext{
${ }^{1}$ If the expression for spectral radiance were derived from eq. (10.141) without making the large-distance approximation of eq. (10.142) the formula would depend upon $R$. Since this is incompatible with classical radiometry it is, strictly speaking, necessary to define radiance as the far field limit of the derivative of the fluence rate:

$$
L_{\lambda}(x, y, \theta, \phi)=\operatorname{Lim}_{R \rightarrow \infty} \frac{\partial}{\partial \omega} F_{t, \lambda}^{\prime}\left(x^{\prime}, y^{\prime}\right)
$$

where $R$, as given by eq. (10.139), is the distance from the source point ( $x, y)$ to the detector point $\left(x^{\prime}, y^{\prime}\right)$, and $F_{t, \lambda}^{\prime}\left(x^{\prime}, y^{\prime}\right)=w_{\lambda}^{\prime}\left(x^{\prime}, y^{\prime} ; x^{\prime}, y^{\prime}\right)$ as usual. In this way $L_{\lambda}$ will depend only upon the direction $(\theta, \phi)$ from the point $(x, y)$. In our derivation we have effectively taken this limit when we make the approximation leading to eq. (10.142). 


$$
\begin{aligned}
L_{\lambda}^{\prime}\left(x^{\prime}, y^{\prime}, \theta, \phi\right) & =\frac{z^{\prime}}{R \lambda^{2}} \iint_{\text {source }} w_{\lambda}\left(x+\frac{3}{2} \xi, y+\frac{1}{2} \eta ; x-\frac{2}{2} \xi, y-\frac{1}{2} \eta\right) \\
& \cdot \exp \left\{-\frac{2 \pi i}{\lambda R} \cdot\left[\xi \cdot\left(x^{\prime}-x\right)+\eta \cdot\left(y^{\prime}-y\right)\right]\right\} \cdot d \xi \cdot d \eta
\end{aligned}
$$

In this equation, $\theta$ and $\phi$ specify the direction from the source point ( $x, y$ ) to the detector point $\left(x^{\prime}, y^{\prime}\right)$ :

$$
\begin{aligned}
\cos \theta & =z^{\prime} / R \\
\sin \theta \cdot \cos \phi & =\left(x^{\prime}-x\right) / R \\
\sin \theta \cdot \sin \phi & =\left(y^{\prime}-y\right) / R
\end{aligned}
$$

If we use these relations to eliminate the primed coordinates and remember that geometrically radiance is invariant along a ray we obtain eq. (10.20)

$$
\begin{aligned}
L_{\lambda}(x, y, \theta, \phi)= & \frac{\cos \theta}{\lambda^{2}} \cdot \iint_{\text {source }} w_{\lambda}\left(x+\frac{1}{2} \xi, y+\frac{1}{2} \eta ; x-\frac{1}{2} \xi, y-\frac{1}{2} \eta\right) \\
& \cdot \exp \left[-\frac{2 \pi i}{\lambda} \cdot \sin \theta \cdot(\xi \cdot \cos \phi+\eta \cdot \sin \phi)\right] \cdot d \xi \cdot d \eta \cdot
\end{aligned}
$$

This is the spectral radiance at the source point $(x, y)$ in the direction $(\theta, \phi)$. The integration covers the entire source plane, or beam cross-section if the "source" plane is not a primary source. We see that the cross-spectral density over the entire source plane contributes to the spectral radiance at any one point of the source. This is consistent with the idea that, by diffraction, all points in the source can contribute something to the signal received by a radiometer nominally aimed at a single point.

The spectral irradiance at the point $\left(x^{\prime}, y^{\prime}, z^{\prime}\right)$, irradiated by a source centered at the origin can be obtained similarly from the relation \{eq. (4.20) [10.9]\} between irradiance and fluence rate:

$$
\mathrm{dE}^{\prime}\left(\mathrm{x}^{\prime}, \mathrm{y}^{\prime}, \theta, \phi\right)=\cos \theta_{\mathrm{r}} \cdot \mathrm{dF} \mathrm{t}^{\prime}\left(\mathrm{x}^{\prime}, \mathrm{y}^{\prime}, \theta, \phi\right)
$$

Using eq. (10.144) for the element of spectral fluence rate we obtain

$$
\begin{aligned}
& E_{\lambda}\left(x^{\prime}, y^{\prime}, z^{\prime}\right)=\iiint \int_{\text {source }} w_{\lambda}\left(x+\frac{1}{2} \xi, y+\frac{1}{2} \eta ; x-\frac{1}{2} \xi, y-\frac{1}{2} \eta\right) \\
& \quad \exp \left\{-\frac{2 \pi i}{\lambda R} \cdot\left[\xi \cdot\left(x^{\prime}-x\right)+\eta \cdot\left(y^{\prime}-y\right)\right]\right\} \cdot \frac{z^{\prime 2}}{R^{4} \lambda^{2}} \cdot \cos \theta_{r} \cdot d \xi \cdot d \eta \cdot d x \cdot d y \cdot
\end{aligned}
$$

which differs from eq. (10.143) for spectral fluence rate only by the extra factor cos ${ }_{r}$. The angle $\theta_{r}$ is the angle between the normal to the irradiated surface at ( $x^{\prime}, y^{\prime}$ ) and the direction to the source point $(x, y)$. If the irradiated plane is parallel to the source plane then $\cos \theta_{r}=\cos \theta=z^{\prime} / R$. In both eqs. (10.143) and (10.151) $R$ is a function of $x$ and $y$ [eq. (10.139)] which makes the integrals difficult. If we can assume that the source dimensions are small compared with $\sqrt{\lambda R}$-- the Fraunhofer approximation -- then $\mathrm{R} \approx\left(\mathrm{x}^{\prime}{ }^{2}+\mathrm{y}^{, 2}+\mathrm{z}, 2\right)^{\frac{1}{2}}$ and these equations become: 


$$
\begin{aligned}
F_{t, \lambda}\left(x^{\prime}, y^{\prime}, z^{\prime}\right) & =\frac{\cos ^{2} \theta}{R^{2} \lambda^{2}} \cdot \iiint \int_{\text {source }} w_{\lambda}\left(x+\frac{1}{2} \xi, y+\frac{3}{2} n ; x-\frac{1}{2} \xi, y-\frac{1}{2} n\right) \\
& \cdot \exp \left[-\frac{2 \pi i}{\lambda \bar{R}} \cdot\left(\xi \cdot x^{\prime}+\eta \cdot y^{\prime}\right)\right] \cdot d x \cdot d y \cdot d \xi \cdot d \eta
\end{aligned}
$$

and

$$
E_{\lambda}\left(x^{\prime}, y^{\prime}, z^{\prime}\right)=\cos \theta_{r} \cdot F_{t, \lambda}\left(x^{\prime}, y^{\prime}, z^{\prime}\right)
$$

where $\cos \theta=z^{\prime} / R$ and both the $x, y$ and $\xi, \eta$ integrations independently cover the source plane.

The rest of the common radiometric quantities can now be easily expressed in terms of the cross-spectral density. From eqs. (10.16), (10.143), and (10.148) we find

$$
\begin{aligned}
I_{\lambda}(\theta, \phi) & =\frac{\cos ^{2} \theta}{\lambda^{2}} \cdot \iiint \int_{\text {source }} w_{\lambda}\left(x+\frac{1}{2} \xi, y+\frac{1}{2} n ; x-\frac{1}{2} \xi, y-\frac{1}{2} \eta\right) \\
& \cdot \exp \left[-\frac{2 \pi i}{\lambda} \cdot \sin \theta \cdot(\xi \cdot \cos \phi+\eta \cdot \sin \phi)\right] \cdot d x \cdot d y \cdot d \xi \cdot d \eta
\end{aligned}
$$

for the spectral radiant intensity of the source in the direction $(\theta, \phi)$. The spectral radiant exitance from the source plane is obtained by integrating the spectral radiance [eq. (10.149)] over the hemisphere with respect to projected $\operatorname{solid}$ angle $\cos \theta \cdot d \omega=\cos \theta \cdot \sin \theta \cdot d \theta \cdot d \phi$. The integrations over $\theta$ and $\phi$ can be carried out to give

$$
\begin{aligned}
M_{\lambda}(x, y) & =\iint_{\text {source }} W_{\lambda}\left(x+\frac{1}{2} \xi, y+\frac{1}{2} n ; x-\frac{1}{2} \xi, y-\frac{1}{2} n\right) \\
& \cdot \frac{2 \pi}{\lambda^{2}} \cdot\left[\frac{\sin \left(\frac{2 \pi}{\lambda} \cdot \sqrt{\xi^{2}+n^{2}}\right)}{\left(\frac{2 \pi}{\lambda} \cdot \sqrt{\xi^{2}+n^{2}}\right)^{3}}-\frac{\cos \left(\frac{2 \pi}{\lambda} \cdot \sqrt{\xi^{2}+n^{2}}\right)}{\left(\frac{2 \pi}{\lambda} \cdot \sqrt{\xi^{2}+n^{2}}\right)^{2}}\right] \cdot d \xi \cdot d \eta
\end{aligned}
$$

For highly incoherent fields, these expressions for the radiometric quantities possess all the properties of the corresponding classically defined radiometric quantities. However, for fields possessing significant coherence they possess all the right properties except that the spectral radiance and spectral radiant exitance in some circumstances can be negative and can be non-zero in regions of a source plane outside of the physically defined source area itself. As we've indicated these quirks arise because these are classical geometrical optics concepts which are not strictly definable in the real world of wave optics. Fortunately it appears that these deficiencies aren't likely to arise in most cases of interest [10.14] so that eq. (10.149) and eqs. (10.152) to (10.155) can be accepted as the appropriate extension of classical radiometry to include coherence.

We illustrate the calculation of the classical radiometric quantities from the crossspectral density by an example of a source whose cross-spectral density function is given by

$$
w_{\lambda}\left(x_{1}, y_{1} ; x_{2}, y_{2}\right)=A_{0}^{2} \cdot \exp \left(-\frac{x^{2}+y^{2}}{2 \sigma^{2}}\right) \cdot \operatorname{sinc}\left(\frac{2 \beta}{\lambda} \cdot \sqrt{\xi^{2}+n^{2}}\right)
$$


where $x, y, \xi$ and $n$ are the average and difference coordinates defined in eqs. (10.138). The spectral fluence rate in the source plane is a Gaussian function of the distance from the origin:

$$
F_{t, \lambda}(x, y)=A_{0}^{2} \cdot \exp \left(-r^{2} / 2 \sigma^{2}\right)
$$

where $r^{2}=x^{2}+y^{2}$. The degree of coherence in the source plane is a sinc function of the distance between the two points whose coherence is being considered:

$$
\mu\left(x_{1}, y_{1} ; x_{2}, y_{2}\right)=\operatorname{sinc}\left(\frac{2 \beta}{\lambda} \cdot \rho\right)
$$

where $\rho^{2}=\xi^{2}+n^{2}=\left(x_{1}-x_{2}\right)^{2}+\left(y_{1}-y_{2}\right)^{2}$. We see that the parameter $\beta$ determines the coherence interval in the source and the parameter $\sigma$ is a measure of the size of the source. Since this is clearly a quasihomogeneous source, because the cross-spectral density is a product of a function of the average coordinates, $x$ and $y$, and a function of the difference coordinates, $\xi$ and $n$, (this implies that $\sigma \gg \lambda / \beta$ ), let us first simplify the expressions for the classical radiometric quantities by applying them to a quasihomogeneous source. Upon substituting $F_{t, \lambda}(x, y) \cdot g(\xi, \eta)$ for the cross-spectral density in eqs. (10.149), (10.152), and $(10.155)$ we obtain:

$$
\begin{aligned}
& L_{\lambda}(x, y, \theta, \phi)=\frac{\cos \theta}{\lambda^{2}} \cdot F_{t, \lambda}(x, y) \cdot \iint_{\text {source }} g(\xi, \eta) \\
& \text { - } \exp \left[-\frac{2 \pi i}{\lambda} \cdot \sin \theta \cdot(\xi \cdot \cos \phi+\eta \cdot \sin \phi)\right] \cdot d \xi \cdot d \eta \text {, } \\
& F_{t, \lambda}\left(x^{\prime}, y^{\prime}, z^{\prime}\right)=\left(\frac{z^{\prime}}{\lambda R^{2}}\right)^{2} \cdot N_{\lambda} \cdot \iint_{\text {source }} g(\xi, n) \cdot \exp \left[-\frac{2 \pi i}{\lambda} \cdot\left(\xi \cdot x^{\prime}+\eta \cdot y^{\prime}\right)\right] \cdot d \xi \cdot d n,
\end{aligned}
$$

and

$$
\begin{aligned}
M_{\lambda}(x, y)=F_{t, \lambda}(x, y) & \iint_{\text {source }} g(\xi, n) \cdot \frac{2 \pi}{\lambda^{2}} \\
& \cdot\left[\frac{\sin \left(\frac{2 \pi}{\lambda} \cdot \sqrt{\xi^{2}+n^{2}}\right)}{\left(\frac{2 \pi}{\lambda} \cdot \sqrt{\xi^{2}+n^{2}}\right)^{3}}-\frac{\cos \left(\frac{2 \pi}{\lambda} \cdot \sqrt{\xi^{2}+n^{2}}\right)}{\left(\frac{2 \pi}{\lambda} \cdot \sqrt{\xi^{2}+n^{2}}\right)^{2}}\right] \cdot d \xi \cdot d n,
\end{aligned}
$$

where

$$
\begin{aligned}
& N_{\lambda}=\iint_{\text {source }} F_{t, \lambda}(x, y) \cdot d x \cdot d y, \\
& \phi=\tan ^{-1}(y / x)
\end{aligned}
$$

and $\theta=\cos ^{-1}\left(z^{\prime} / R\right)$. The incident spectral irradiance at a distance $R$ from the source and the spectral radiant intensity of that source are obtained from the spectral fluence rate at that distance by multiplying by $\cos \theta_{r}$ and $R^{2}$, respectively [eqs. (10.153) and (10.16)], where ${ }^{\theta}$ is the angle between the normal to the irradiated surface at $\left(x^{\prime}, y^{\prime}\right)$ and the direction 
to the origin in the source plane. If the irradiated surface is parallel to the source plane then ${ }_{r} r=\theta$. The spectral flux is obtained by integrating the spectral exitance $M_{\lambda}(x, y)$ over the source area to give:

$$
\Phi_{\lambda}=\mathrm{N}_{\lambda} \cdot \iint_{\text {source }} g(\xi, n) \cdot \frac{2 \pi}{\lambda^{2}} \cdot\left[\frac{\sin \left(\frac{2 \pi}{\lambda} \cdot \sqrt{\xi^{2}+n^{2}}\right)}{\left(\frac{2 \pi}{\lambda} \cdot \sqrt{\xi^{2}+n^{2}}\right)^{3}}-\frac{\cos \left(\frac{2 \pi}{\lambda} \cdot \sqrt{\xi^{2}+n^{2}}\right)}{\left(\frac{2 \pi}{\lambda} \cdot \sqrt{\xi^{2}+n^{2}}\right)^{2}}\right] \cdot d \xi \cdot d n .
$$

Now with $F_{t, \lambda}(x, y)$ given by eq. $(10.157)$ and $g(\xi, n)=\mu$ given by eq. (10.158) the evaluation of the integrals occurring in eqs. (10.159) to (10.162) can be carried out with the help of a table of integrals [10.15], [10.16], [10.17]). We find:

$$
\mathrm{N}_{\lambda}=2 \pi \mathrm{A}_{0}^{2} \cdot \sigma^{2},
$$

the integral appearing in eqs. (10.159) and (10.160) is

$$
\frac{\lambda^{2}}{2 \pi \beta \cdot \sqrt{\beta^{2}-\sin ^{2} \theta}} \text { for } \sin \theta<\beta \text {, and } 0 \text { otherwise, }
$$

and the integral in eqs. $(10.161)$ and $(10.162)$ is

$$
\frac{1}{2}\left[1+\frac{1-\beta^{2}}{2 \beta} \cdot \ln \left(\frac{1+\beta}{|1-\beta|}\right)\right]
$$

Thus we finally obtain the following radiometric quantities for the source whose cross-spectral density is given by eq. $(10.156)$ :

$$
\begin{aligned}
& L_{\lambda}(x, y, \theta, \phi)= \begin{cases}A_{0}^{2} \cdot \exp \left(-r^{2} / 2 \sigma^{2}\right) \cdot \frac{\cos \theta}{2 \pi \beta \cdot \sqrt{\beta^{2}-\sin ^{2} \theta}} & \text { for } \sin \theta<\beta \\
0 & \text { otherwise }\end{cases} \\
& F_{t, \lambda}\left(x^{\prime}, y^{\prime}, z^{\prime}\right)= \begin{cases}2 \pi A_{0}^{2} \cdot \sigma^{2} \cdot \frac{z^{\prime 2}}{2 \pi \beta \cdot R^{4} \sqrt{\beta^{2}-1+z^{\prime 2} / R^{2}}} & \text { for }\left(1-z^{\prime 2} / R^{2}\right)<\beta^{2} \\
0 & \text { otherwise }\end{cases} \\
& M_{\lambda}(x, y)=A_{0}^{2} \cdot \exp \left(-r^{2} / 2 \sigma^{2}\right) \cdot \frac{1}{2}\left[1+\frac{1-\beta^{2}}{2 \beta} \cdot \ln \left(\frac{I+B}{|I-B|}\right)\right]
\end{aligned}
$$

$$
E_{\lambda}\left(x^{\prime}, Y^{\prime}, z^{\prime}\right)= \begin{cases}2 \pi A_{0}^{2} \cdot \sigma^{2} \cdot \frac{z^{\prime^{2} \cdot \cos \theta} r}{2 \pi \beta \cdot R^{4} \cdot \sqrt{\beta^{2} \cdot 1+z^{\prime 2} / R^{2}}} & \text { for }\left(1-z^{\prime 2} / R^{2}\right)<\beta^{2} \\ 0 & \text { otherwise }\end{cases}
$$




$$
\begin{aligned}
& I_{\lambda}(\theta, \phi)= \begin{cases}2 \pi A_{0}^{2} \cdot \sigma^{2} \cdot \frac{\cos ^{2} \theta}{2 \pi \beta \cdot \sqrt{\beta^{2}-\sin ^{2} \theta}} & \text { for } \sin \theta<\beta \\
0 & \text { otherwise }\end{cases} \\
& \Phi_{\lambda}=2 \pi A_{0}^{2} \cdot \sigma^{2} \cdot \frac{1}{2}\left[1+\frac{1-\beta^{2}}{2 \beta} \cdot \ln \left(\frac{1+\beta}{|1-\beta|}\right)\right] .
\end{aligned}
$$

When $\beta=1$ the expression for spectral radiance [eq. (10.166)] becomes independent of direction:

$$
L_{\lambda}(x, y, \theta, \phi)=A_{0}^{2} \cdot \exp \left(-r^{2} / 2 \sigma^{2}\right) /(2 \pi)
$$

This, then, is a Lambertian source and we see that the degree of coherence of a Lambertian source, such as a blackbody, must resemble [from eq. (10.158) with $\beta=1$ ]

$$
\mu=\sin (2 \pi \rho / \lambda) /(2 \pi \rho / \lambda)
$$

with coherence interval (if we use the criterion $|\mu|=\frac{1}{2}$ ) of the order of $0.3 \lambda$. This is presumably about as incoherent as any real source can be. Notice that the coherence interval of a Lambertian source depends upon the observing wavelength rather than upon any parameter characteristic of the source. The other radiometric quantities for a quasihomogeneous Lambertian source are readily obtained from eqs. (10.167) to (10.171) by setting $\beta=1$ :

$$
\begin{aligned}
& F_{t, \lambda}\left(x^{\prime}, y^{\prime}, z^{\prime}\right)=A_{0}^{2} \cdot \sigma^{2} \cdot z^{\prime} / R^{3} \\
& M_{\lambda}(x, y)=A_{0}^{2} \cdot \exp \left(-r^{2} / 2 \sigma^{2}\right) / 2 \\
& E_{\lambda}\left(x^{\prime}, Y^{\prime}, z^{\prime}\right)=A_{O}^{2} \cdot \sigma^{2} \cdot \cos \theta_{r^{\prime}} z^{\prime} / R^{3} \\
& I_{\lambda}(\theta, \phi)=A_{0}^{2} \cdot \sigma^{2} \cdot \cos \theta \\
& \Phi_{\lambda}=A_{O}^{2} \cdot \sigma^{2} \cdot \pi \cdot
\end{aligned}
$$

In these expressions $R^{2}=x^{\prime 2}+y^{\prime 2}+z^{\prime 2}, r^{2}=x^{2}+y^{2}$, and $\theta=\cos ^{-1}\left(z^{\prime} / R\right)$.

Although in pursuing this example we have made no mathematical approximations there is an inconsistency in that we have treated a source of infinite extent using equations derived for a finite source [(eqs. (10.149) to (10.155)]. Fortunately the Gaussian approaches zero so fast as $r$ increases that the error is negligible as long as $\sigma$ is very much smaller than the observation distance $R$.

POLARIZATION. The theory of partial coherence given in this chapter is known as a scalar theory because it treats a single component of the electric field vector as though the field in other directions didn't exist. A more complete theory includes consideration of the coherence between, say, the $x$-component of the field at point $P_{1}$ and the $y$-component of the field at point $\mathrm{P}_{2}$. Thus, one replaces the cross-spectral density function with a $2 \times 2$ matrix of four such functions: 


$$
w_{\lambda}{ }^{(j, k)}\left(P_{1}, P_{2}\right)=\frac{1}{\Delta \lambda} \cdot\left\langle V^{*}{ }_{j}\left(P_{1}+\Delta P, t\right) \cdot V_{k}\left(P_{2}+\Delta P, t\right)\right\rangle
$$

where $j$ and $k$ can each be either $x$ or $y$ and $V_{j}(P, t)$ is the complex representation of the $j$ component of the electric field at the point $P$ as seen through a narrow $(\Delta \lambda)$ pass-band spectral filter or monochromator. The angular brackets again denote an average over time and over identical small areas around $\mathrm{P}_{1}$ and $\mathrm{P}_{2}$ as the small displacement $\Delta \mathrm{P}$ covers the measurement resolution area. In terms of these cross-spectral density matrix elements the Stokes-vector components [10.20] of spectral fluence rate are:

$$
\begin{aligned}
& F_{t, \lambda, 0}(P)=w_{\lambda}^{(x, x)}(P, P)+w_{\lambda}^{(y, y)}(P, P) \\
& F_{t, \lambda, 1}(P)=w_{\lambda}^{(x, x)}(P, P)-w_{\lambda}^{(y, y)}(P, P) \\
& F_{t, \lambda, 2}(P)=w_{\lambda}^{(x, y)}(P, P)+w_{\lambda}^{(y, x)}(P, P) \\
& F_{t, \lambda, 3}(P)=i \cdot\left[w_{\lambda}^{(y, x)}(P, P)-w_{\lambda}^{(x, y)}(P, P)\right] .
\end{aligned}
$$

If $v_{j}(P, t)$ is written as

$$
V_{j}(P, t)=A_{j}(P, t) \cdot \exp \left[i \gamma_{j}(P, t)-2 \pi i v t\right]
$$

where $v=c / \lambda$, then

$$
W_{\lambda}{ }^{(j, k)}(P, P)=\frac{1}{\Delta \lambda} \cdot\left\langle A_{j}(P, t) \cdot A_{k}(P, t) \cdot \exp \left[i \gamma_{k}(P, t)-i \gamma_{j}(P, t)\right]\right\rangle
$$

and the stokes components of spectral fluence rate become:

$$
\begin{aligned}
& F_{t, \lambda, 0}(P)=\frac{1}{\Delta \lambda} \cdot\left\langle A_{x}{ }^{2}+A_{y}{ }^{2}\right\rangle \\
& F_{t, \lambda, 1}(P)=\frac{1}{\Delta \lambda} \cdot\left\langle A_{x}{ }^{2}-A_{y}{ }^{2}\right\rangle \\
& F_{t, \lambda, 2}(P)=\frac{2}{\Delta \lambda} \cdot\left\langle A_{x} \cdot A_{y} \cdot \cos \left(\gamma_{x}-\gamma_{y}\right)\right\rangle \\
& F_{t, \lambda, 3}(P)=\frac{2}{\Delta \lambda} \cdot\left\langle A_{x} \cdot A_{y} \cdot \sin \left(\gamma_{x}-\gamma_{y}\right)\right\rangle .
\end{aligned}
$$

These equations are equivalent to eq. (6.3) [10.20]. Thus, polarization is included as an essential aspect of a more general coherence theory with the stokes parameters being one possible set of four linear combinations of the four cross-spectral density function matrix elements. Although there are circumstances involving polarization in which the scalar theory of coherence is clearly inadequate [10.21], it appears that a complete theory would probably only be required in exceptional radiometric situations where precise calculations of significant coherence effects are needed. 
SUMMARY of CHAPTER 10. The field quantity which, in partially coherent fields, plays a basic role somewhat analogous to that of spectral radiance in incoherent fields is the cross-spectral density, $w_{\lambda}\left(x_{1}, y_{1}, z_{1} ; x_{2}, y_{2}, z_{2}\right)$. It is a complex-valued function of the coordinates of two field points. The only direct interest radiometrists have in this function is in the special case where both sets of coordinates describe the same point. Then the cross-spectral density reduces to the spectral fluence rate at that point (Chapter 4, page 17 [10.9]):

$$
W_{\lambda}(x, y, z ; x, y, z)=F_{t, \lambda}(x, y, z)
$$

Otherwise its importance for radiometry is that if one follows the propagation of crossspectral density from a source through an optical system with the calculation of fluence rate or one of the other radiometric quantities of geometric optics only at the final detector, then all coherence effects such as diffraction will be properly accounted for. The propagation law for cross-spectral density is:

$$
W_{\lambda}\left(P_{1}^{\prime}, P_{2}^{\prime}\right)=\iint_{\text {source }} W_{\lambda}\left(P_{1}, P_{2}\right) \cdot \frac{\exp \left[2 \pi i \cdot\left(s_{1}-s_{2}\right) / \lambda\right] \cdot \cos \theta_{1} \cdot \cos \theta_{2}}{s_{1} \cdot s_{2} \cdot \lambda^{2}} \cdot d P_{1} \cdot d P_{2} \cdot
$$

Thus if the cross-spectral density function $W_{\lambda}\left(P_{1}, P_{2}\right)$ is known for all pairs of points $P_{1}$ and $\mathrm{P}_{2}$ in one surface or cross-section of a beam, which we can regard as a source, and if the optical path length $s_{1}$ for a ray from $P_{1}$ to $P_{1}^{\prime}$ and the optical path length $s_{2}$ from $\mathrm{P}_{2}$ to $\mathrm{P}_{2}^{\prime}$ are known for all points $\mathrm{P}_{1}$ and $\mathrm{P}_{2}$ in the source, then this multiple integral over the source surface permits the cross-spectral density to be calculated at the point pair $P_{1}^{\prime}, P_{2}^{\prime}$. The angles $\theta_{i}$ are the angles between the normal to the source surface at $P_{i}$ and the direction to $P_{i}^{\prime}$, where $i$ is either 1 or 2 . For a complicated optical system this equation must be applied sequentially between each successive pair of beam limiting apertures with the results of one calculation serving as the source for the next stage of the calculation. The only approximation of any consequence made in deriving this equation is that the source dimensions as well as all distances $s_{1}$ and $s_{2}$ involved must be much larger than the wavelength $\lambda$.

In the evaluation of the propagation integrals [eq. (10.36)], it is desirable to perform as much of the integration analytically as possible. To this end it is usual to restrict the surface containing $P_{1}$ and $P_{2}$ to the $X-Y$ plane $(z=0)$ and to expand the expressions for $s_{1}$ and $s_{2}$ in power series in $l / R_{1}$ and $1 / R_{2}$ where $R_{1}$ is the distance from the origin to $\mathrm{P}_{1}^{\prime}$, and $\mathrm{R}_{2}$ is the distance from the origin to $\mathrm{P}_{2}^{\prime}$. If we assume that the source dimensions are small compared to $\sqrt{\lambda R_{i}}$ and retain only the most important terms of these expansions we obtain the result:

$$
\begin{aligned}
W_{\lambda}\left(x_{1}^{\prime}, y_{1}^{\prime}, z_{1}^{\prime} ; x_{2}^{\prime}, y_{2}^{\prime}, z_{2}^{\prime}\right)= & \frac{z_{1}^{\prime} z_{2}^{\prime}}{R_{1}^{2} R_{2}^{2} \lambda^{2}} \cdot \exp \left[\frac{2 \pi i}{\lambda} \cdot\left(R_{1}-R_{2}\right)\right] \cdot \iiint \int_{\text {source }} w_{\lambda}\left(x_{1}, y_{1} ; x_{2}, y_{2}\right) \\
& \cdot \exp \left[-\frac{2 \pi i}{\lambda}\left(\frac{x_{1} x_{1}^{\prime}+y_{1} y_{1}^{\prime}}{R_{1}}-\frac{x_{2} x_{2}^{\prime}+y_{2} Y_{2}^{\prime}}{R_{2}}\right)\right] \cdot d x_{1} \cdot d y_{1} \cdot d x_{2} \cdot d y_{2} \cdot
\end{aligned}
$$

Although this condition on the source size is often not fulfilled, the approximation (Fraunhofer approximation) may be useful anyway in radiometry if the goal is merely to estimate the magnitude of small measurement errors which diffraction may introduce. 
One situation in which the sequential application of the propagation equation (10.36) is not overly difficult is in simple lens (or focusing mirror) image formation. If the crossspectral density function $\mathrm{W}_{\lambda}\left(\mathrm{P}_{1}, \mathrm{P}_{2}\right)$ is known at all point pairs in the object plane then the cross-spectral density in the image plane is given by:

$$
\begin{aligned}
\mathrm{w}_{\lambda}\left(\mathrm{x}_{1}^{\prime \prime}, \mathrm{y}_{1}^{\prime \prime}, \mathrm{z}_{1}^{\prime \prime} ; \mathrm{x}_{2}^{\prime \prime}, \mathrm{y}_{2}^{\prime \prime}, \mathrm{z}_{2}^{\prime \prime}\right) & =\frac{1}{\mathrm{R}^{2} \mathrm{R}^{\prime \prime}{ }_{\lambda}^{4}} \cdot \iiint_{\text {object }} \mathrm{w}_{\lambda}\left(\mathrm{x}_{1}, \mathrm{y}_{1} ; \mathrm{x}_{2}, \mathrm{y}_{2}\right) \\
& \cdot \mathrm{K}_{\lambda}\left(\mathrm{x}_{1}, \mathrm{y}_{1} ; \mathrm{x}_{1}^{\prime \prime}, \mathrm{y}_{1}^{\prime \prime}\right) \cdot \mathrm{K}_{\lambda}^{\star}\left(\mathrm{x}_{2}, \mathrm{y}_{2} ; \mathrm{x}_{2}^{\prime \prime}, \mathrm{y}_{2}^{\prime \prime}\right) \cdot \mathrm{dx_{1 }} \cdot \mathrm{dy}_{1} \cdot \mathrm{dx}_{2} \cdot \mathrm{dy}_{2} \cdot
\end{aligned}
$$

In this expression $R$ is the distance from the object to the lens and $R$ " from the lens to the image. The function $K_{\lambda}\left(P, P^{\prime \prime}\right)$ is the transmission function of the optical system. It is given by an integral over the lens aperture plane:

$$
K\left(x, y ; x^{\prime \prime}, y^{\prime \prime}\right)=\iint_{-\infty}^{\infty} P\left(x^{\prime}, y^{\prime}\right) \cdot \exp \left[\frac{2 \pi i}{\lambda} \cdot\left(s+s^{\prime \prime}-R-R^{\prime \prime}\right)\right] \cdot d x^{\prime} \cdot d y^{\prime} \cdot
$$

Here $s$ is the optical path length for a ray from point $(x, y)$ in the object plane to point $\left(x^{\prime}, y^{\prime}\right)$ in the lens aperture plane and $s^{\prime \prime} i s, s i m i l a r l y$, the optical path length for a ray from the point $\left(x^{\prime}, y^{\prime}\right)$ to the image plane point $\left(x^{\prime \prime}, y^{\prime \prime}\right)$. Since $s$ and $s^{\prime \prime}$ depend upon these points, to be complete we should write them as $s\left(x, y^{\prime} x^{\prime}, y^{\prime}\right)$ and $s^{\prime \prime}\left(x^{\prime}, y^{\prime} ; x^{\prime \prime}, y^{\prime \prime}\right)$. The factor $P\left(x^{\prime}, y^{\prime}\right)$ is the pupil function for the aperture. In its simplest form it merely indicates where the aperture is transparent and where it is opaque by taking on the value 1 within the aperture and the value 0 elsewhere. In more complicated situations the pupil function can indicate regions of partial transparency by assuming intermediate values between 0 and 1 and it can indicate regions of phase retardation by assuming complex values, although this latter condition can alternatively be accounted for in the calculations of $s$ and $s^{\prime \prime}$. For perfect imagery and a transparent rectangular aperture, $K_{\lambda}(P, P ") \quad$ reduces to

$$
\mathrm{K}_{\lambda}\left(\mathrm{x}, \mathrm{y} ; \mathrm{x}^{\prime \prime}, \mathrm{y}^{\prime \prime}\right)=4 \mathrm{a}^{\prime} \mathrm{b}^{\prime} \cdot \frac{\sin \left[\frac{2 \pi \mathrm{a}^{\prime}}{\lambda} \cdot\left(\frac{\mathrm{x}}{\mathrm{R}}+\frac{\mathrm{x}^{\prime \prime}}{\mathrm{R}^{\prime \prime}}\right)\right]}{\frac{2 \pi \mathrm{a}^{\prime}}{\lambda} \cdot\left(\frac{\mathrm{x}}{\mathrm{R}}+\frac{\mathrm{x}^{\prime \prime}}{\mathrm{R}^{\prime \prime}}\right)} \cdot \frac{\sin \left[\frac{2 \pi \mathrm{b}^{\prime}}{\lambda} \cdot\left(\frac{\mathrm{Y}}{\mathrm{R}}+\frac{\mathrm{Y}^{\prime \prime}}{\mathrm{R}^{\prime \prime}}\right)\right]}{\frac{2 \pi \mathrm{b}^{\prime}}{\lambda} \cdot\left(\frac{\mathrm{Y}}{\mathrm{R}}+\frac{\mathrm{Y}^{\prime \prime}}{\mathrm{R}^{\prime \prime}}\right)}
$$

where the aperture width is $2 a^{\prime}$ in the $x$-direction and $2 b^{\prime}$ in the $y-d i r e c t i o n$. Eq. (10.178) substituted into eq. (10.133) yields eq. (10.99), the equation for perfect imaging with a transparent rectangular lens aperture.

All of the classical radiometric quantities can be expressed in terms of the crossspectral density. Equation (10.15a) illustrates the simplest of these relationships -- that for spectral fluence rate. Some of the others are:

Spectral radiance:

$$
\begin{aligned}
L_{\lambda}(x, y, \theta, \phi)= & \frac{\cos \theta}{\lambda^{2}} \cdot \iint_{\text {source }} w_{\lambda}\left(x+\frac{1}{2} \xi, y+\frac{1}{2} n ; x-\frac{1}{2} \xi, y-\frac{1}{2} n\right) \\
& \cdot \exp \left[-\frac{2 \pi i}{\lambda} \cdot \sin \theta \cdot(\xi \cdot \cos \phi+\eta \cdot \sin \phi)\right] \cdot d \xi \cdot d \eta \cdot
\end{aligned}
$$




$$
\begin{aligned}
& E_{\lambda}\left(x^{\prime}, y^{\prime}, z^{\prime}\right)=\iiint \int_{\text {source }} w_{\lambda}\left(x+\frac{1}{2} \xi, y+\frac{1}{2} n ; x-\frac{1}{2} \xi, y-\frac{1}{2} n\right) \\
& \quad \exp \left\{-\frac{2 \pi i}{\lambda R} \cdot\left[\xi \cdot\left(x^{\prime}-x\right)+n \cdot\left(y^{\prime}-y\right)\right]\right\} \cdot \frac{z^{\prime 2}}{R^{4} \lambda^{2}} \cdot \cos \theta_{r} \cdot d \xi \cdot d n \cdot d x \cdot d y \cdot
\end{aligned}
$$

\section{Spectral radiant intensity:}

$$
\begin{aligned}
I_{\lambda}(\theta, \phi)= & \frac{\cos ^{2} \theta}{\lambda^{2}} \cdot \iiint \int_{\text {source }} w_{\lambda}\left(x+\frac{1}{2} \xi, y+\frac{1}{2} \eta ; x-\frac{1}{2} \xi, y-\frac{1}{2} \eta\right) \\
& \cdot \exp \left[-\frac{2 \pi i}{\lambda} \cdot \sin \theta \cdot(\xi \cdot \cos \phi+\eta \cdot \sin \phi)\right] \cdot d x \cdot d y \cdot d \xi \cdot d \eta
\end{aligned}
$$

Spectral radiant exitance:

$$
\begin{aligned}
M_{\lambda}(x, y) & =\iint_{\text {source }} W_{\lambda}\left(x+\frac{1}{2} \xi, y+\frac{1}{2} n ; x-\frac{1}{2} \xi, y-\frac{1}{2} n\right) \\
& \cdot \frac{2 \pi}{\lambda^{2}} \cdot\left[\frac{\sin \left(\frac{2 \pi}{\lambda} \cdot \sqrt{\xi^{2}+n^{2}}\right)}{\left(\frac{2 \pi}{\lambda} \cdot \sqrt{\xi^{2}+n^{2}}\right)^{3}}-\frac{\cos \left(\frac{2 \pi}{\lambda} \cdot \sqrt{\xi^{2}+n^{2}}\right)}{\left(\frac{2 \pi}{\lambda} \cdot \sqrt{\xi^{2}+n^{2}}\right)^{2}}\right] \cdot d \xi \cdot d \eta
\end{aligned}
$$

In these equations the point $(x, y)$ is a point in a plane (which we call a source but may be any cross-section of the beam) where the cross-spectral density is assumed completely known. $\xi$ and $n$ are displacements from this point. The primed coordinates refer to a point, which is irradiated by the source, at a distance $R$ from the source point $(x, y)$, and $\theta_{r}$ is the angle between the normal to the irradiated surface at $\left(x^{\prime}, y^{\prime}\right)$ and the direction to the point $(x, y)$. It is possible to imagine coherent fields for which $L_{\lambda}$ calculated from the expression above will not agree with our classical ideas of acceptable behavior for spectral radiance -- by being negative, for example. The reason for this is that spectral radiance is inherently a geometrical optics concept: its definition is equivalent to requiring that the position and momentum of a photon be simultaneously determinable with arbitrary accuracy, which, of course, is forbidden by the Heisenberg uncertainty principle. Fortunately such nonclassical behavior of $\mathrm{L}_{\lambda}$ should rarely occur in practice. Although the above expressions for spectral radiant intensity and spectral radiant exitance are both derived from that for spectral radiance, only the latter suffers from this same deficiency.

The cross-spectral density of a uniform rectangular incoherent source is usually taken to be something like:

$$
\begin{aligned}
\mathrm{w}_{\lambda}\left(\mathrm{x}_{1}, \mathrm{y}_{1} ; \mathrm{x}_{2}, \mathrm{y}_{2}\right)= & \lambda^{2} \mathrm{~A}_{\mathrm{O}}^{2} \cdot \delta\left(\mathrm{x}_{1}-\mathrm{x}_{2}\right) \cdot \delta\left(\mathrm{y}_{1}-\mathrm{y}_{2}\right) \\
& \text { for }\left|\mathrm{x}_{1}\right|,\left|\mathrm{x}_{2}\right|<\mathrm{a} \text { and }\left|\mathrm{y}_{1}\right|,\left|\mathrm{y}_{2}\right|<\mathrm{b}
\end{aligned}
$$

and

$$
w_{\lambda}\left(x_{1}, y_{1} ; x_{2}, y_{2}\right)=0 \text { otherwise. }
$$


This expression lends itself to easy evaluation of integrals such as eq. (10.42) or

eq. (10.133) and thereforeiis widely used in spite of some shortcomings (e.g., it implies that $\left.F_{t, \lambda}=\infty\right)$. The opposite extreme of a uniform rectangular coherent source has a cross-spectral density given by:

$$
\begin{aligned}
W_{\lambda}\left(x_{1}, y_{1} ; x_{2}, y_{2}\right)= & A_{0}^{2} \\
& \text { for }\left|x_{1}\right|,\left|x_{2}\right|<a \text { and }\left|y_{1}\right|,\left|y_{2}\right|<b \\
= & 0 \text { otherwise, }
\end{aligned}
$$

where, for simplicity, uniform phase across the source is assumed [ $\theta_{0}=0$ in eq. (10.59)]. An idea of the consequences of coherence in a radiometric measurement may usually be obtained by considering these two source cross-spectral density functions in the sequence of expressions of the form of eqs. (10.42) and (10.133) which describe the measurement geometry up to the detector. At the detector the irradiance (or, simpler, and almost equivalently, the fluence rate) can be calculated, and if the results for both kinds of sources when considered in the over-all measurement process (e.g., including calibrations) show the same behavior over the range of source sizes and distances, spectral distributions, etc., to be encountered then it is safe to assume that coherence will not affect these measurements. On the other hand if the results show that the two types of sources behave differently over some sub-range of source characteristics then it may be possible to change the measurement geometry to avoid the problem or, as a last resort, it may be possible by more elaborate measurements and careful calculations to correct the radiometer results for coherence.

Another expression for the cross-spectral density function, which is frequently introduced as an approximate description of many fields encountered in radiometry -- especially those originating in incandescent sources, is

$$
w_{\lambda}\left(x+\frac{1}{2} \xi, y+\frac{1}{2} \eta ; x-\frac{1}{2} \xi, y-\frac{1}{2} \eta\right)=F_{t, \lambda}(x, y) \cdot g(\xi, n)
$$

This is known as the quasihomogeneous model and is appropriate for regions of a field where the fluence rate $F_{t, \lambda}(x, y)$ can be assumed constant over areas the size of the coherence area. The function $g(\xi, \eta)$ is simply the (complex) degree of coherence of the field. The use of this model considerably simplifies the propagation integral eq. (10.42) and, consequently, also the integrals for the classical radiometric quantities [eqs. (10.149, 10.151, 10.154 , and 10.155$)]$. 
References

[10.1] Fred E. Nicodemus, Henry J. Kostkowski, and Albert T. Hattenburg, "Introduction", Chapter 1 of "Self-Study Manual on Optical Radiation Measurements: Part I-Concepts", Nat. Bur. Stand. (U.S.) Tech. Note 910-1, 93 pages (Mar. 1976) pp. 1-9, see esp. p. 8 .

[10.2] Max Born and Emil Wolf, "Principles of Optics" 6th (rev.) ed. (Pergamon Press, 1980); especially Chap. VIII "Elements of the Theory of Diffraction", Chap. IX "The Diffraction Theory of Aberrations", and Chap. X "Interference and Diffraction with Partially Coherent Light".

[10.3] Arvind S. Marathay, "Elements of Optical Coherence Theory" (John Wiley \& Sons, New York, 1982).

[10.4] Ari T. Friberg, "Effects of coherence in radiometry", Opt. Eng., Vol. 21, No. 5 (Sept./Oct. 1982), pp. 927-936.

[10.5] George O. Reynolds and John B. DeVelis, "Review of optical coherence effects in instrument design", Part I, Opt. Eng. Vol. 20, No. 3 (May/June 1981), pp. SR-084 to SR-094; Part II, Ibid., No. 4 (July/Aug. 1981), pp. SR-124 to SR-130; Part III, Ibid, No. 5 (Sept./Oct. 1981), pp. SR-166 to SR-172.

[10.6] Klaus D. Mielenz, "Gas Lasers and Conventional Sources in Interferometry", in "Electron Beam and Laser Beam Technology", L. Marton and A. B. El-Kareh, eds. Supplement 4 to "Advances in Electronics and Electron Physics" L. Marton and Claire Marton, eds. (Academic Press, New York, 1968), pp. 89-137.

[10.7] Emil Wolf, "Coherence and Radiometry", J. Opt. Soc. Am., Vol. 68, No. 1 (Jan. 1978) pp. 6-17.

[10.8] H. P. Baltes, J. Geist, and A. Walther, "Radiometry and Coherence", Chap. 5 in "Inverse Source Problems in Optics", H. P. Baltes, ed. (Springer-Verlag, Berlin, 1978), pp. 119-154.

[10.9] Fred E. Nicodemus, "More on the Distribution of Optical Radiation with Respect to Position and Direction", Chapter 4 of "Self-Study Manual on Optical Radiation Measurements: Part I--Concepts". Nat. Bur. Stand. (U.S.), Tech. Note 910-2, 118 pages (Feb. 1978) pp. 1-57.

[10.10] "International Lighting Vocabulary, 3rd ed. common to the CIE and IEC", Publication CIE No. 17 (E-1.1) 1970, Bureau Central de la CIE, $4 \mathrm{Av}$. du Recteur Poincaré, 75-Paris 16\%, France. [Available from: Dr. Klaus D. Mielenz, Secretary, U.S. National Committee, CIE, National Bureau of Standards, Washington, D.C. 20234.] (A 4 th edition is in preparation.)

[10.11] Ronald N. Bracewell, "The Fourier Transform and Its Applications" 2nd ed. (McGraw-Hil1, 1978); Chap. 5 "The Impulse Symbol", pp. 69-97.

[10.12] L. P. Boivin, "Diffraction corrections in the radiometry of extended sources", Appl. Optics, Vol. 15, No. 5, (May 1976), pp. 1204-1209.

[10.13] Ari T. Friberg, "Radiation from partially coherent sources", Opt. Eng., Vol. 21, No. 2 (Mar./Apr. 1982), pp. 362-369.

[10.14] Ari T. Friberg, "On the Generalized Radiance Associated with Radiation from a Quasihomogeneous Planar Source", Optica Acta, Vol. 28, No. 2, (1981), pp. 261-277.

[10.15] Milton Abramowitz and Irene A. Stegun, eds., "Handbook of Mathematical Functions with Formulas, Graphs, and Mathematical Tables", Nat. Bur. Stand. (U.S.), Appl. Math. Ser. 55; June 1964 (7th printing, May 1968, with corrections), $1060 \mathrm{pp}$.

[10.16] Arthur Erdélyi, ed., "Tables of Integral Transforms, Vols. I \& II". (McGraw-Hill, 1954).

[10.17] I. S. Gradshteyn and I. M. Ryzhik, "Table of Integrals, Series, and Products (corrected and enlarged)". (Academic Press, 1980).

[10.18] Klaus D. Mielenz, "Spectroscope Slit Images in Partially Coherent Light", J. Opt. Soc. Am., Vol. 57, No. 1 (Jan. 1967), pp. 66-74. 
[10.19] Fred E. Nicodemus and Henry J. Kostkowski, "Distribution of Optical Radiation with Respect to Position and Direction -- Radiance", Chapter 2 of "SelfStudy Manual on Optical Radiation Measurements: Part I--Concepts", Nat. Bur. Stand. (U.S.), Tech. Note 910-1, 93 pages (March 1976) pp. 10-44).

[10.20] John B. Shumaker, "Distribution of Optical Radiation with Respect to Polarization", Chapter 6 of "Self-Study Manual on Optical Radiation Measurements: Part I--Concepts", Nat. Bur. Stand. (U.S.), Tech Note 910-3, 62 pages (June 1977).

[10.21] William H. Carter, "Properties of electromagnetic radiation from a partially correlated current distribution", J. Opt. Soc. Am., Vol. 70, No. 9, (Sept. 1980), pp. 1067-1074. 
NBS-114A (REV. 2-8C)

U.S. DEPT. OF COMM.

BIBLIOGRAPHIC DATA

SHEET (See instructions)

1. PUBLICATION OR

REPORT NO.

NBS TN 910-6

March 1983

4. TITLE AND SUBTITLE

SELF-STUDY MANUAL ON OPTICAL RADIATION MEASUREMENTS, Part I--Concepts

Chapter 10. Introduction to Coherence in Radiometry

5. AUTHOR(S)

John B. Shumaker

6. PERFORMING ORGANIZATION (If joint or other than NBS, see instructions)

7. Contract Grant No.

NATIONAL BUREAU OF STANDARDS

DEPARTMENT OF COMMERCE

WASHINGTON, D.C. 20234

8. Type of Report \& Period Covered

Final

9. SPONSORING ORGANIZATION NAME AND COMPLETE ADDRESS (Street. City, Stote, ZIF)

Same as Item 6.

10. SUPPLEMENTARY NOTES

Document describes a computer program; SF-185, FIPS Software Summary, is attached.

11. ABSTRACT (A 200-word or less factual summary of most significant information. If document includes a significant bibliography or literature survey, mention it here)

This is the sixth in a series of Technical Notes (910-) entitled "Self-Study Manual on Optical Radiation Measurements". It contains Chapter 10 of Part I of this Manual. Additional chapters will continue to be published, similarly, as they are completed. The Manual is a comprehensive tutorial treatment of the measurement of optical radiation that is complete enough for self instruction. Detailed chapter summaries make it also a convenient authoritative reference source.

In this chapter we introduce the reader to the basic field quantity of the theory of partial coherence, the cross-spectral density function, and show how it is used to describe radiation fields. We discuss the propagation of cross-spectral density along a beam and illustrate this propagation with calculations of diffraction effects in a number of simple aperturing and imaging examples. For instance, we treat in considerable detail one of the most common radiometric situations in which coherence effects can manifest themselves, the measurement of the slit-scattering function of a monochromator. Among other things, this treatment shows that laser illumination of a monochromator entrance slit must be nearly on-axis to avoid serious slit-scattering function distortions. Finally, we present the relationships between the cross-spectral density function and the classical radiometric quantities, such as spectral radiance.

12. KEY WORDS (Six to twelve entries: alphabetical order: capitalize only proper names; and separate key words by semicolons)

Coherence; cross-spectral density; incoherence; interference;

partial coherence; radiometry; spectroradiometry; wave optics.

\section{AVAILABILITY}

Enlimited

$\square$ For Official Distribution. Do Not Release to NTIS

[Xrder From Superintendent of Documents, U.S. Government Printing Office, Washington, D.C. 20402.

Order From National Technical Information Service (NTIS), Springfield, VA. 2216I

14. NO. OF

PRINTED PAGES

69

15. Price

$\$ 5.00$ 



\section{NBS TECHNICAL PUBLICATIONS}

\section{PERIODICALS}

LRNAL OF RESEARCH-The Journal of Research of the wonal Bureau of Standards reports NBS research and developnt in those disciplines of the physical and engineering sciences in ch the Bureau is active. These include physics, chemistry, ineerıng. mathematics, and computer sciences. Papers cover a ad range of subjects, with major emphasis on measurement thodology and the basic technology underlying standardization. so included from time to time are survey articles on topics sely related to the Bureau's technical and scientific programs. a special service to subscribers each issue contains complete tions to all recent Bureau publications in both NBS and nonS media. Issued six times a year. Annual subscription: domestic 8: foreign $\$ 22.50$. Single copy, $\$ 5.50$ domestic; $\$ 6.90$ foreign.

\section{NONPERIODICALS}

onographs-Major contributions to the technical literature on rous subjects related to the Bureau's scientific and technical actues.

andbooks-Recommended codes of engineering and industrial actice (Including safety codes) developed in cosperation with inrested industries, professional organizations, and regulatory idies.

pecial Publications-Include proceedings of conferences sponred by NBS, NBS annual reports, and other special publications propriate to this grouping such as wall charts, pocket cards, and bliographies.

oplied Mathematics Series_Mathematical tables, manuals, and udies of special interest to physicists, engineers, chemists, ologists, mathematicians, computer programmers, and others gaged in scientific and technical work.

ational Standard Reference Data Series-Provides quantitative lat on the physical and chemical properties of materials, comled from the world's literature and critically evaluated. eveloped under a worldwide program coordinated by NBS under e authority of the National Standard Data Act (Public Law $1-396)$.

OTE: The principal publication outlet for the foregoing data is e Journal of Physical and Chemical Reference Data (JPCRD) tblished quarterly for NBS by the American Chemical Society (CS) and the American Institute of Physics (AIP). Subscriptions, prints, and supplements available from ACS, 1155 Sixteenth St., W. Washington, DC 20056
Building Science Series-Disseminates technical information developed at the Bureau on building materials, components, systems, and whole structures. The series presents research results, test methods, and performance criteria related to the structural and environmental functions and the durability and safety characteristics of building elements and systems.

Technical Notes-Studies or reports which are complete in themselves but restrictive in their treatment of a subject. Analogous to monographs but not so comprehensive in scope or definitive in treatment of the subject area. Often serve as a vehicle for final reports of work performed at NBS under the sponsorship of other government agencies.

Voluntary Product Standards-Developed under procedures published by the Department of Commerce in Part 10. Title 15, of the Code of Federal Regulations. The standards establish nationally recognized requirements for products, and provide all concerned interests with a basis for common understanding of the characteristics of the products. NBS administers this program as a supplement to the activities of the private sector standardizing organizations.

Consumer Information Series-Practical information, based on NBS research and experience, covering areas of interest to the consumer. Easily understandable language and illustrations provide useful background knowledge for shopping in today's technological marletplace.

Order the above NBS publications from: Superintendent of Documents. Government Printing Office, Washington. DC 20402.

Order the following NBS publications-FIPS and NBSIR's-from the National Technical Information Service, Springfield, VA 22161.

Federal Information Processing Standards Publications (FIPS PUB)-Publications in this series collectively constitute the Federal Information Processing Standards Register. The Register serves as the official source of information in the Federal Government regarding standards issued by NBS pursuant to the Federal Property and Admınistrative Services Act of 1949 as amended. Public Law 89.306 (79 Stat. 1127), and as implemented by Executive Order 11717 (38 FR 12315, dated May 11, 1973) and Part 6 of Title 15 CFR (Code of Federal Regulations).

NBS Interagency Reports (NBSIR) - A special series of interim or final reports on work performed by NBS for outside sponsors (both government and non-government). In general, initial distribution is handled by the sponsor; public distribution is by the National Technical Information Service, Springfield, VA 22161, in paper copy or microfiche form. 
U.S. Department of Commerce

National Bureau of Standards

Washington, D.C. 20234

Official Business

U.S.MAIL

Penalty for Private Use $\$ 300$

POSTAGE AND FEES

U.S. DEPARTMENT OF CO COM-215

THIRD CLASS

BULK RATE 